\title{
CMA-ES and Advanced Adaptation Mechanisms
}

\author{
Youhei Akimoto1 \& Nikolaus Hansen² \\ 1. University of Tsukuba, Japan \\ 2. Inria, Research Centre Saclay, France
}

akimoto@cs.tsukuba.ac.jp nikolaus.hansen@inria.fr

\footnotetext{
Permission to make digital or hard copies of part or all of this work for personal or classroom use is granted without fee provided that copies are not made or distributed for profit or commercial advantage and that copies bear this notice and the full citation on the first page. Copyrights for third-party components of this work must be honored. For all other uses, contact the Owner/Author.

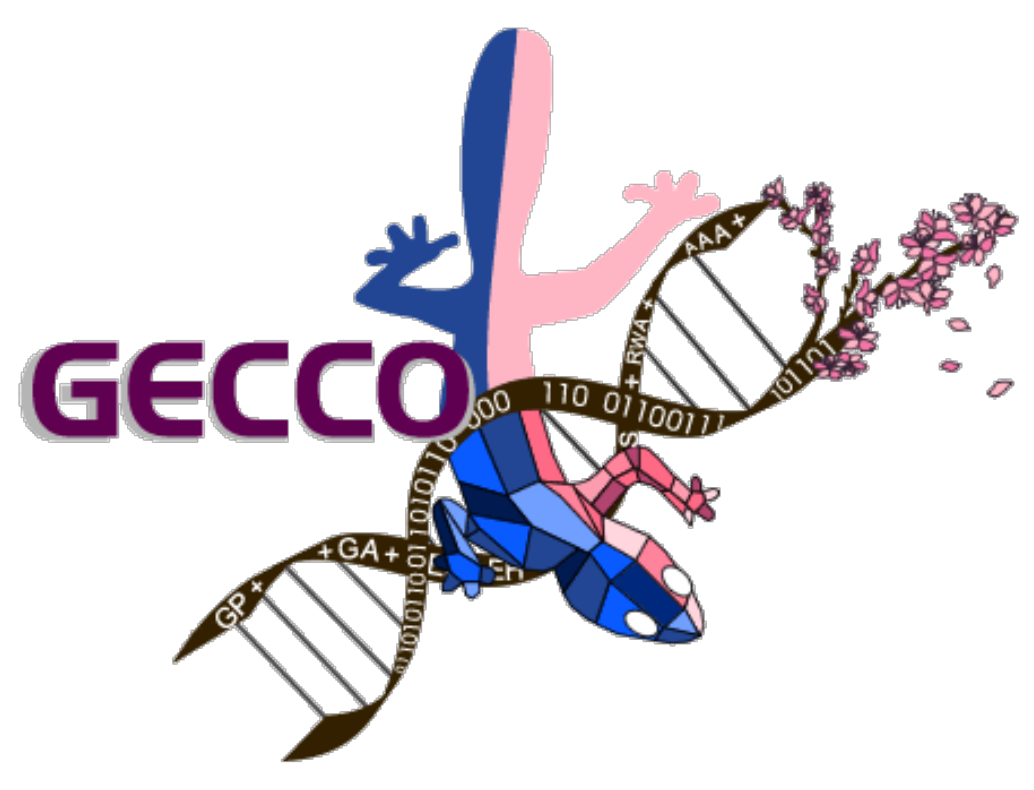


We are happy to answer questions at any time. 


\section{Topics}

1. What makes the problem difficult to solve?

2. How does the CMA-ES work?

- Normal Distribution, Rank-Based Recombination

- Step-Size Adaptation

- Covariance Matrix Adaptation

3. What can/should the users do for the CMA-ES to work effectively on their problem?

- Choice of problem formulation and encoding (not covered)

- Choice of initial solution and initial step-size

- Restarts, Increasing Population Size

- Restricted Covariance Matrix 


\section{Topics}

1. What makes the problem difficult to solve?

2. How does the CMA-ES work?

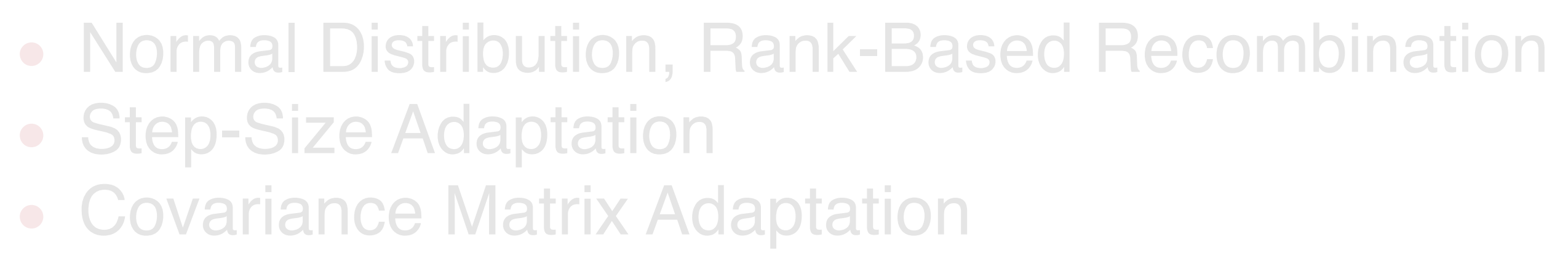

- Choice of problem formulation and encoding (not covered)

- Choice of initial solution and initial step-size

- Restarts, Increasing Population Size

Restricted Covariance Matrix 


\section{Problem Statement}

Continuous Domain Search/Optimization

- Task: minimize an objective function (fitness function, loss function) in continuous domain

$$
f: \mathcal{X} \subseteq \mathbb{R}^{n} \rightarrow \mathbb{R}, \quad \boldsymbol{x} \mapsto f(\boldsymbol{x})
$$

- Black Box scenario (direct search scenario)

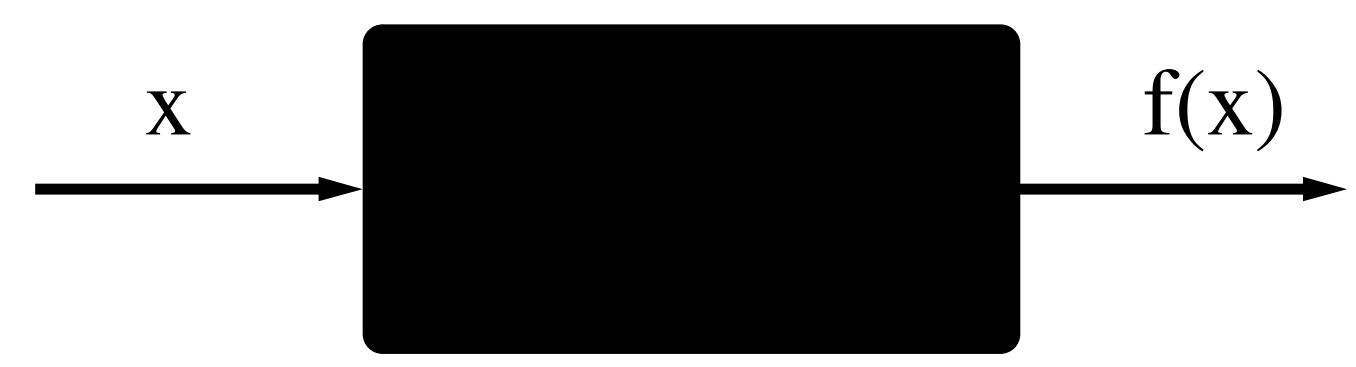

- gradients are not available or not useful

- problem domain specific knowledge is used only within the black box, e.g. within an appropriate encoding

- Search costs: number of function evaluations 


\section{Problem Statement}

Continuous Domain Search/Optimization

- Goal

- fast convergence to the global optimum

- solution $\boldsymbol{x}$ with small function value $f(\boldsymbol{x})$ with least search cost

.. or to a robust solution $x$ there are two conflicting objectives

- Typical Examples

- shape optimization (e.g. using CFD)

curve fitting, airfoils

- model calibration

- parameter calibration controller, plants, images

- Problems

- exhaustive search is infeasible

- naive random search takes too long

- deterministic search is not successful / takes too long

Approach: stochastic search, Evolutionary Algorithms 


\section{What Makes a Function Difficult to Solve?}

Why stochastic search?

- non-linear, non-quadratic, non-convex on linear and quadratic functions much better search policies are available

- ruggedness

non-smooth, discontinuous, multimodal, and/or noisy function

- dimensionality (size of search space)

(considerably) larger than three

- non-separability

dependencies between the objective variables

- ill-conditioning

non-smooth level sets
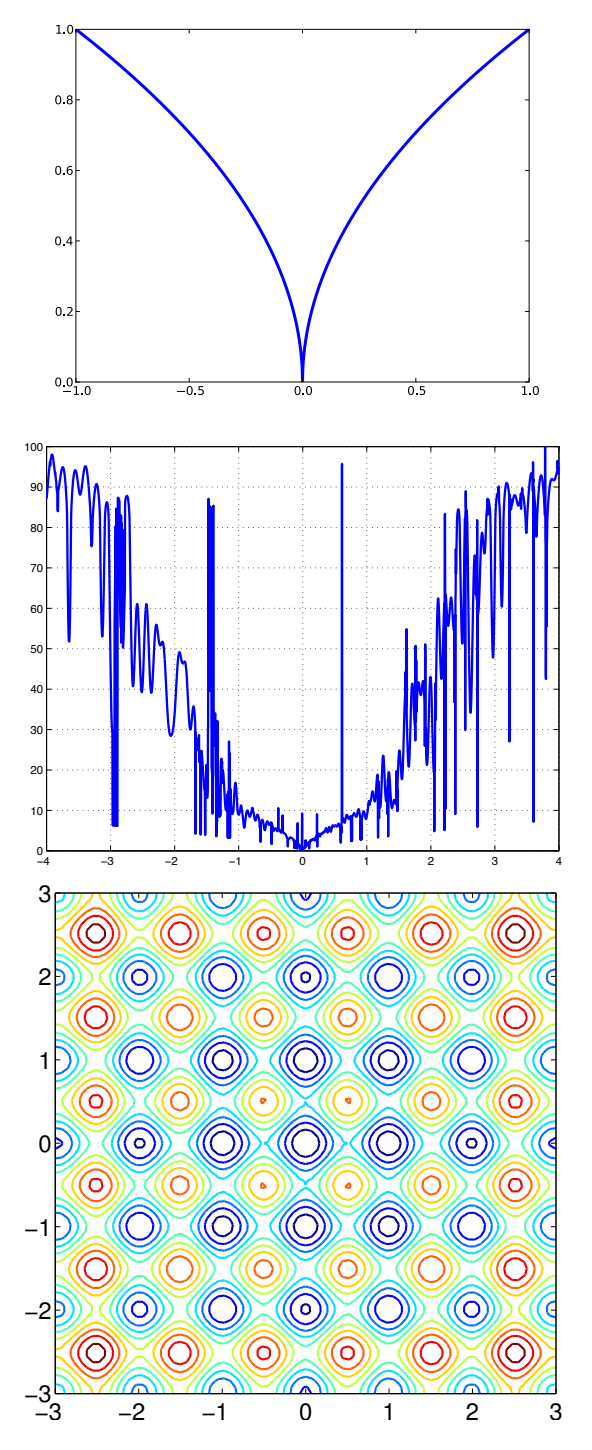

non-smooth level sets 


\section{Ruggedness}

non-smooth, discontinuous, multimodal, and/or noisy

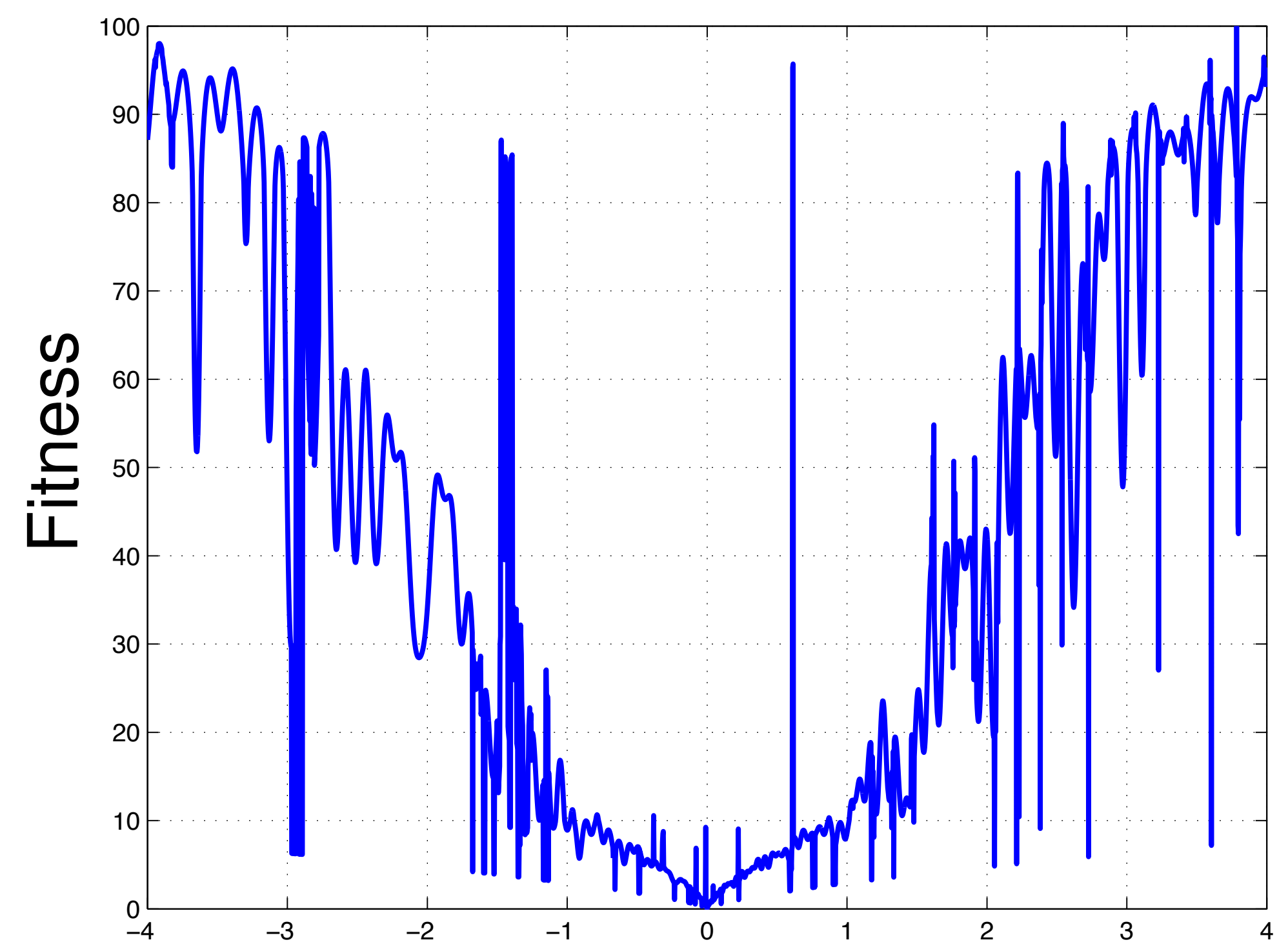

cut from a 5-D example, (easily) solvable with evolution strategies 


\section{Separable Problems}

\section{Definition (Separable Problem)}

A function $f$ is separable if

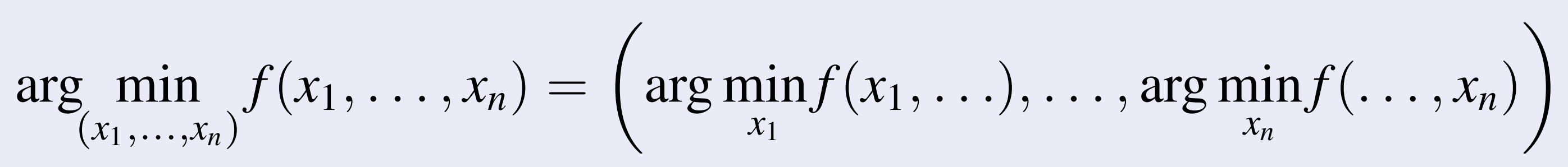

$\Rightarrow$ it follows that $f$ can be optimized in a sequence of $n$ independent 1-D optimization processes

Example: Additively decomposable functions

$$
f\left(x_{1}, \ldots, x_{n}\right)=\sum_{i=1}^{n} f_{i}\left(x_{i}\right)
$$

Rastrigin function

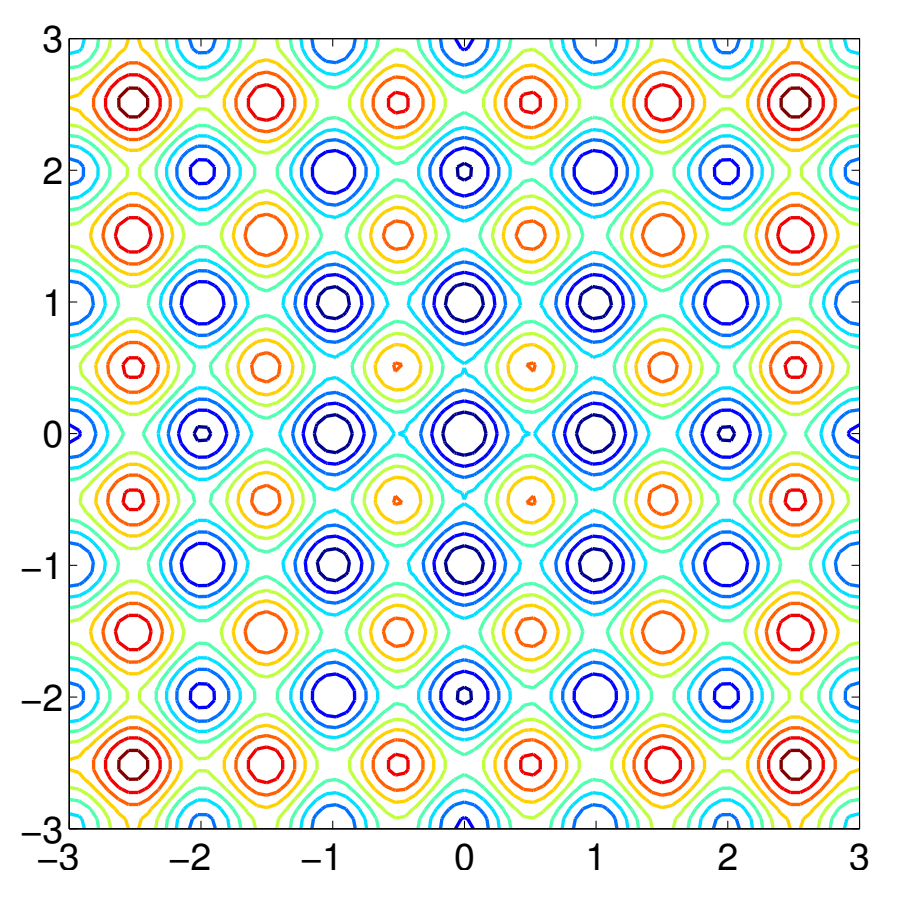




\section{Non-Separable Problems}

Building a non-separable problem from a separable one ${ }^{(1,2)}$

\section{Rotating the coordinate system}

- $f: \boldsymbol{x} \mapsto f(\boldsymbol{x})$ separable

- $f: \boldsymbol{x} \mapsto f(\mathbf{R} \boldsymbol{x})$ non-separable

\section{$\mathbf{R}$ rotation matrix}
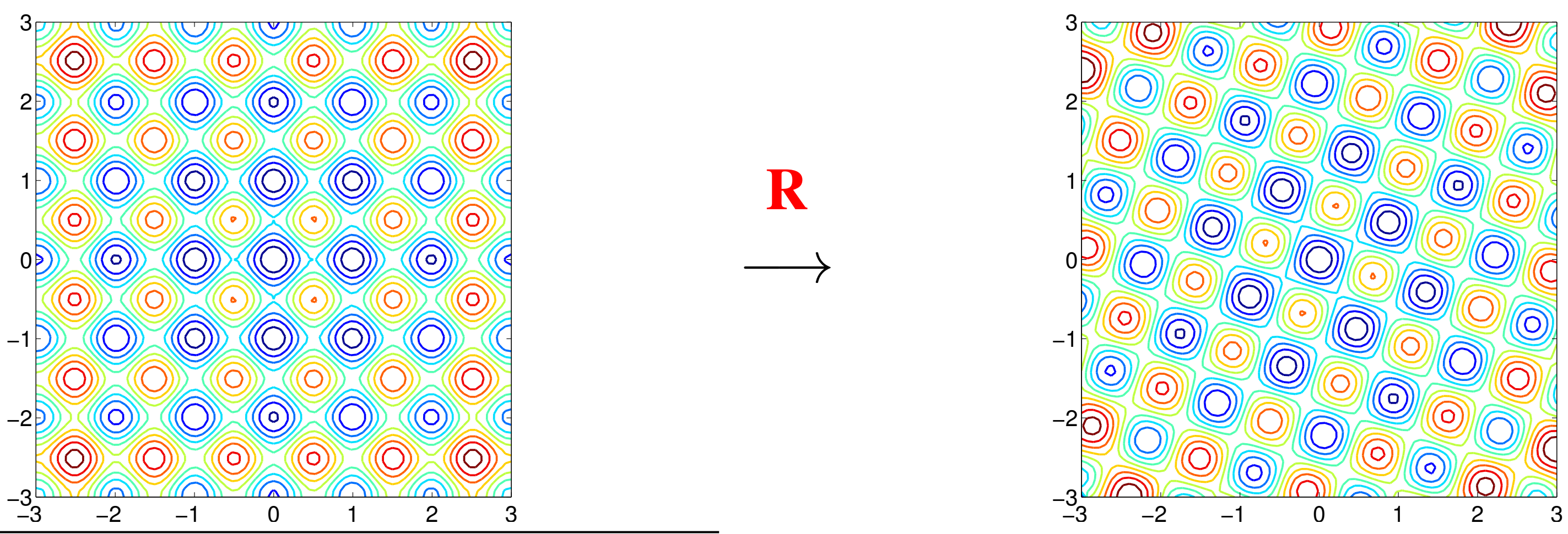

${ }^{1}$ Hansen, Ostermeier, Gawelczyk (1995). On the adaptation of arbitrary normal mutation distributions in evolution strategies: The generating set adaptation. Sixth ICGA, pp. 57-64, Morgan Kaufmann

2 Salomon (1996). "Reevaluating Genetic Algorithm Performance under Coordinate Rotation of Benchmark Functions; A survey of some theoretical and practical aspects of genetic algorithms." BioSystems, 39(3):263-278 


\section{III-Conditioned Problems}

Curvature of level sets

Consider the convex-quadratic function

$f(\boldsymbol{x})=\frac{1}{2}\left(\boldsymbol{x}-\boldsymbol{x}^{*}\right)^{T} \boldsymbol{H}\left(\boldsymbol{x}-\boldsymbol{x}^{*}\right)=\frac{1}{2} \sum_{i} h_{i, i}\left(x_{i}-x_{i}^{*}\right)^{2}+\frac{1}{2} \sum_{i \neq j} h_{i, j}\left(x_{i}-x_{i}^{*}\right)\left(x_{j}-x_{j}^{*}\right)$

$\boldsymbol{H}$ is Hessian matrix of $f$ and symmetric positive definite

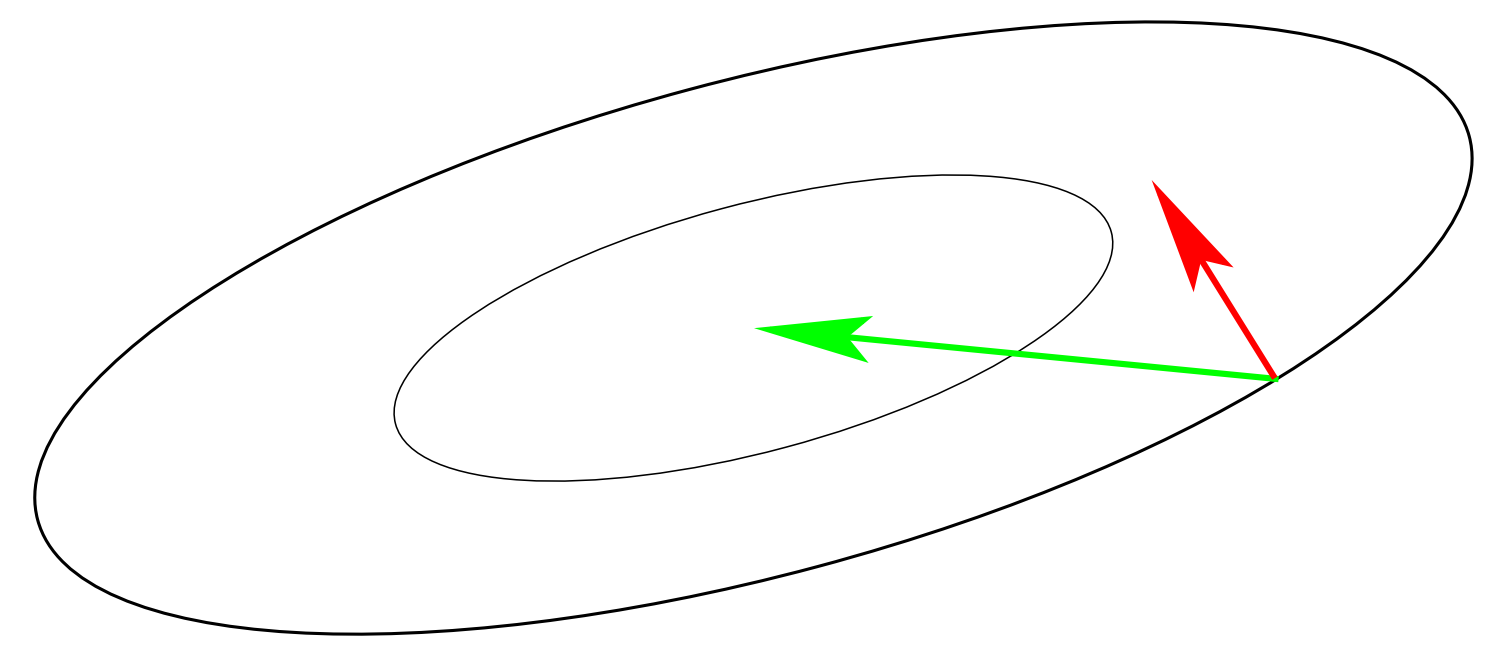

III-conditioning means squeezed level sets (high curvature). Condition number equals nine here. Condition numbers up to $10^{10}$ are not unusual in real world problems.

If $\boldsymbol{H} \approx \mathbf{I}$ (small condition number of $\boldsymbol{H}$ ) first order information (e.g. the gradient) is sufficient. Otherwise second order information (estimation of $\boldsymbol{H}^{-1}$ ) is necessary. 


\section{Non-smooth level sets (sharp ridges)}

Similar difficulty but worse than ill-conditioning

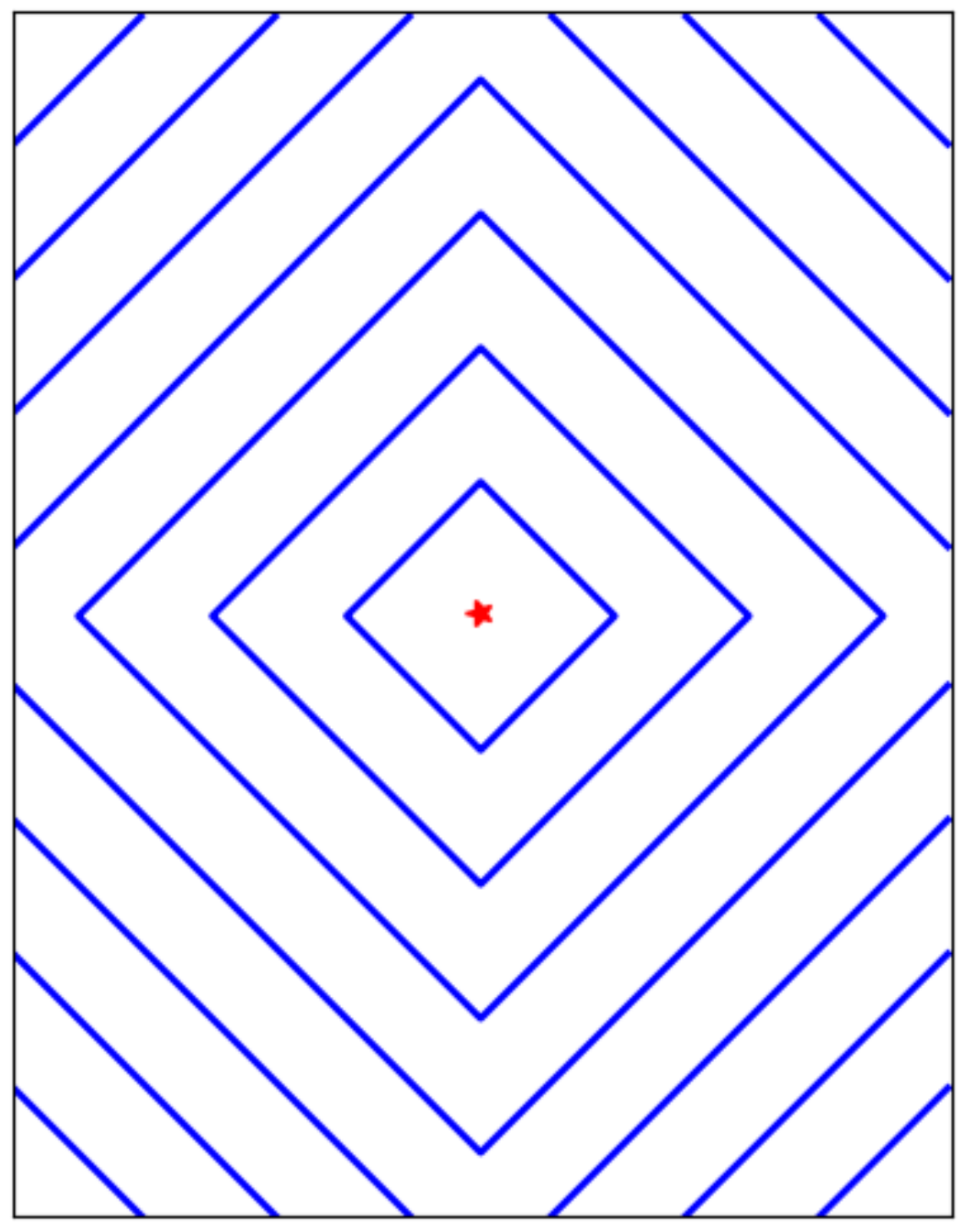

1-norm

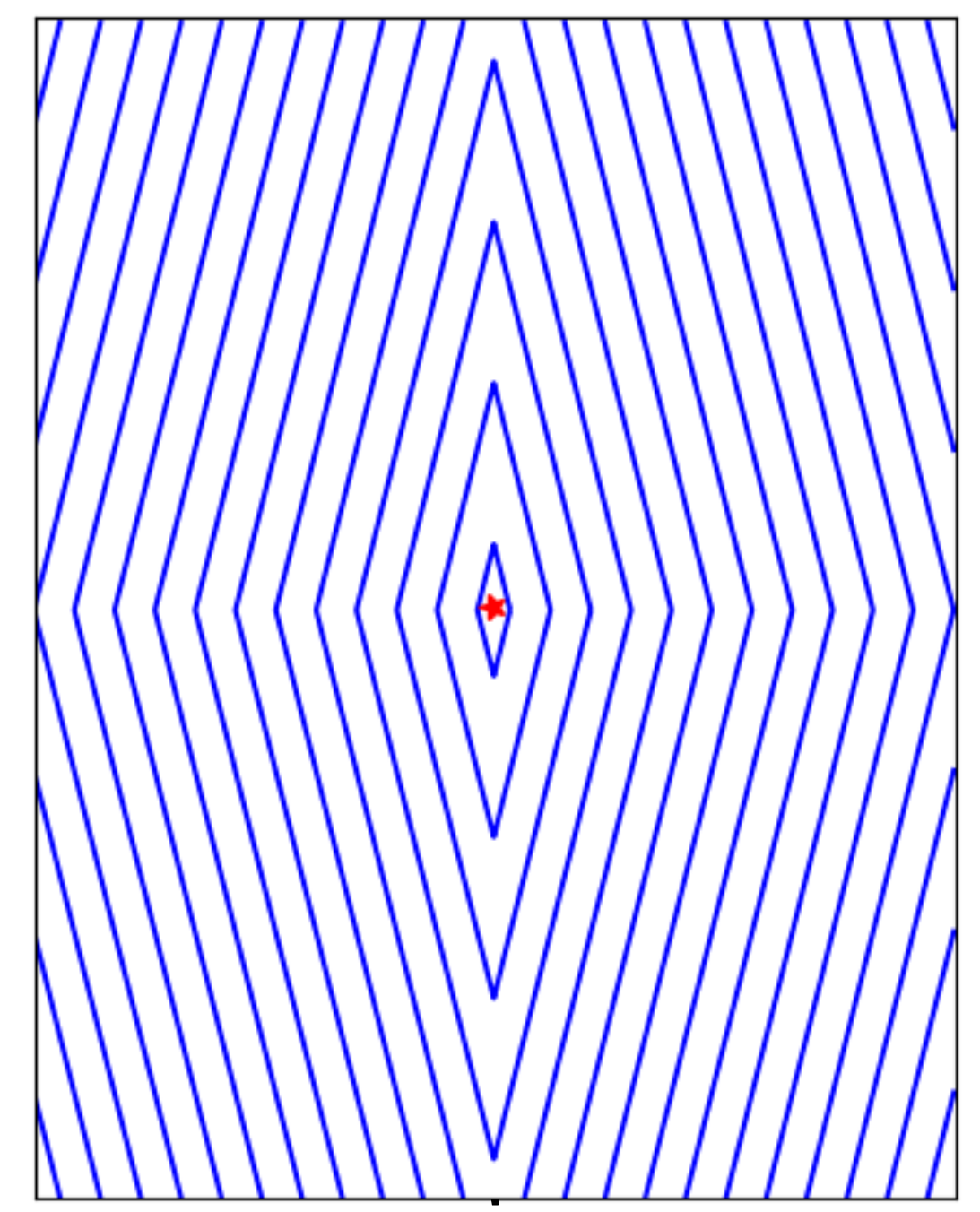

scaled 1-norm

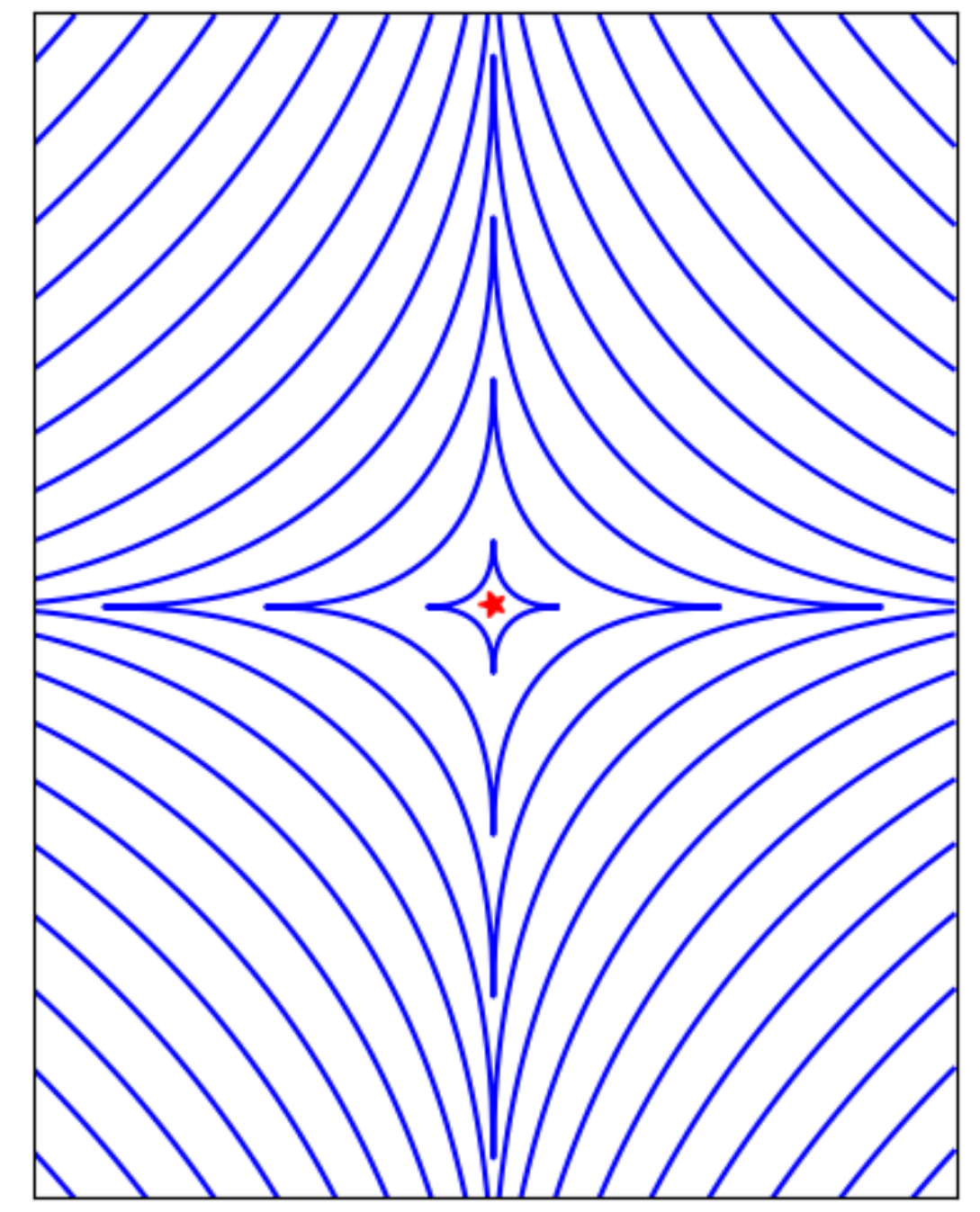

1/2-norm 


\section{What Makes a Function Difficult to Solve?}

... and what can be done

The Problem

Possible Approaches

Dimensionality

III-conditioning

Ruggedness exploiting the problem structure

separability, locality/neighborhood, encoding

second order approach

changes the neighborhood metric

non-local policy, large sampling width (step-size)

as large as possible while preserving a reasonable convergence speed

population-based method, stochastic, non-elitistic recombination operator serves as repair mechanism

restarts 


\section{Topics}

2. How does the CMA-ES work?

- Normal Distribution, Rank-Based Recombination

- Step-Size Adaptation

- Covariance Matrix Adaptation 


\section{Stochastic Search}

A black box search template to minimize $f: \mathbb{R}^{n} \rightarrow \mathbb{R}$

Initialize distribution parameters $\theta$, set population size $\lambda \in \mathbb{N}$ While not terminate

(1) Sample distribution $P(\boldsymbol{x} \mid \theta) \rightarrow \boldsymbol{x}_{1}, \ldots, \boldsymbol{x}_{\lambda} \in \mathbb{R}^{n}$

(2) Evaluate $\boldsymbol{x}_{1}, \ldots, \boldsymbol{x}_{\lambda}$ on $f$

(3) Update parameters $\theta \leftarrow F_{\theta}\left(\theta, \boldsymbol{x}_{1}, \ldots, \boldsymbol{x}_{\lambda}, f\left(\boldsymbol{x}_{1}\right), \ldots, f\left(\boldsymbol{x}_{\lambda}\right)\right)$ 


\section{Stochastic Search}

A black box search template to minimize $f: \mathbb{R}^{n} \rightarrow \mathbb{R}$

Initialize distribution parameters $\theta$, set population size $\lambda \in \mathbb{N}$

While not terminate

(1) Sample distribution $P(\boldsymbol{x} \mid \theta) \rightarrow \boldsymbol{x}_{1}, \ldots, \boldsymbol{x}_{\lambda} \in \mathbb{R}^{n}$

(2) Evaluate $\boldsymbol{x}_{1}, \ldots, \boldsymbol{x}_{\lambda}$ on $f$

(3) Update parameters $\theta \leftarrow F_{\theta}\left(\theta, \boldsymbol{x}_{1}, \ldots, \boldsymbol{x}_{\lambda}, f\left(\boldsymbol{x}_{1}\right), \ldots, f\left(\boldsymbol{x}_{\lambda}\right)\right)$ 


\section{Stochastic Search}

A black box search template to minimize $f: \mathbb{R}^{n} \rightarrow \mathbb{R}$

Initialize distribution parameters $\theta$, set population size $\lambda \in \mathbb{N}$

While not terminate

(1) Sample distribution $P(\boldsymbol{x} \mid \theta) \rightarrow \boldsymbol{x}_{1}, \ldots, \boldsymbol{x}_{\lambda} \in \mathbb{R}^{n}$

(2) Evaluate $\boldsymbol{x}_{1}, \ldots, \boldsymbol{x}_{\lambda}$ on $f$

(3) Update parameters $\theta \leftarrow F_{\theta}\left(\theta, \boldsymbol{x}_{1}, \ldots, \boldsymbol{x}_{\lambda}, f\left(\boldsymbol{x}_{1}\right), \ldots, f\left(\boldsymbol{x}_{\lambda}\right)\right)$ 


\section{Stochastic Search}

A black box search template to minimize $f: \mathbb{R}^{n} \rightarrow \mathbb{R}$

Initialize distribution parameters $\theta$, set population size $\lambda \in \mathbb{N}$ While not terminate

(1) Sample distribution $P(\boldsymbol{x} \mid \theta) \rightarrow \boldsymbol{x}_{1}, \ldots, \boldsymbol{x}_{\lambda} \in \mathbb{R}^{n}$

(2) Evaluate $\boldsymbol{x}_{1}, \ldots, \boldsymbol{x}_{\lambda}$ on $f$

(3) Update parameters $\theta \leftarrow F_{\theta}\left(\theta, \boldsymbol{x}_{1}, \ldots, \boldsymbol{x}_{\lambda}, f\left(\boldsymbol{x}_{1}\right), \ldots, f\left(\boldsymbol{x}_{\lambda}\right)\right)$ 


\section{Stochastic Search}

A black box search template to minimize $f: \mathbb{R}^{n} \rightarrow \mathbb{R}$

Initialize distribution parameters $\theta$, set population size $\lambda \in \mathbb{N}$ While not terminate

(1) Sample distribution $P(\boldsymbol{x} \mid \theta) \rightarrow \boldsymbol{x}_{1}, \ldots, \boldsymbol{x}_{\lambda} \in \mathbb{R}^{n}$

(2) Evaluate $x_{1}, \ldots, x_{\lambda}$ on $f$

(3) Update parameters $\theta \leftarrow F_{\theta}\left(\theta, \boldsymbol{x}_{1}, \ldots, \boldsymbol{x}_{\lambda}, f\left(\boldsymbol{x}_{1}\right), \ldots, f\left(\boldsymbol{x}_{\lambda}\right)\right)$ 


\section{Stochastic Search}

A black box search template to minimize $f: \mathbb{R}^{n} \rightarrow \mathbb{R}$

Initialize distribution parameters $\theta$, set population size $\lambda \in \mathbb{N}$ While not terminate

(1) Sample distribution $P(\boldsymbol{x} \mid \theta) \rightarrow \boldsymbol{x}_{1}, \ldots, \boldsymbol{x}_{\lambda} \in \mathbb{R}^{n}$

(2) Evaluate $\boldsymbol{x}_{1}, \ldots, \boldsymbol{x}_{\lambda}$ on $f$

(3) Update parameters $\theta \leftarrow F_{\theta}\left(\theta, x_{1}, \ldots, x_{\lambda}, f\left(x_{1}\right), \ldots, f\left(x_{\lambda}\right)\right)$ 


\section{Stochastic Search}

A black box search template to minimize $f: \mathbb{R}^{n} \rightarrow \mathbb{R}$

Initialize distribution parameters $\theta$, set population size $\lambda \in \mathbb{N}$

While not terminate

(1) Sample distribution $P(\boldsymbol{x} \mid \theta) \rightarrow \boldsymbol{x}_{1}, \ldots, \boldsymbol{x}_{\lambda} \in \mathbb{R}^{n}$

(2) Evaluate $\boldsymbol{x}_{1}, \ldots, \boldsymbol{x}_{\lambda}$ on $f$

(3) Update parameters $\theta \leftarrow F_{\theta}\left(\theta, \boldsymbol{x}_{1}, \ldots, \boldsymbol{x}_{\lambda}, f\left(\boldsymbol{x}_{1}\right), \ldots, f\left(\boldsymbol{x}_{\lambda}\right)\right)$

Everything depends on the definition of $P$ and $F_{\theta}$

deterministic algorithms are covered as well

In many Evolutionary Algorithms the distribution $P$ is implicitly defined

via operators on a population, in particular, selection, recombination

and mutation

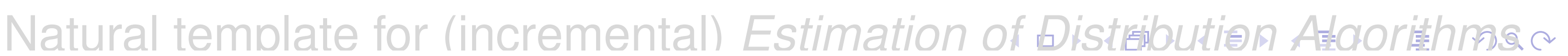




\section{Stochastic Search}

A black box search template to minimize $f: \mathbb{R}^{n} \rightarrow \mathbb{R}$

Initialize distribution parameters $\theta$, set population size $\lambda \in \mathbb{N}$

While not terminate

(1) Sample distribution $P(\boldsymbol{x} \mid \theta) \rightarrow \boldsymbol{x}_{1}, \ldots, \boldsymbol{x}_{\lambda} \in \mathbb{R}^{n}$

(2) Evaluate $\boldsymbol{x}_{1}, \ldots, \boldsymbol{x}_{\lambda}$ on $f$

(3) Update parameters $\theta \leftarrow F_{\theta}\left(\theta, \boldsymbol{x}_{1}, \ldots, \boldsymbol{x}_{\lambda}, f\left(\boldsymbol{x}_{1}\right), \ldots, f\left(\boldsymbol{x}_{\lambda}\right)\right)$

Everything depends on the definition of $P$ and $F_{\theta}$

deterministic algorithms are covered as well

In many Evolutionary Algorithms the distribution $P$ is implicitly defined via operators on a population, in particular, selection, recombination and mutation

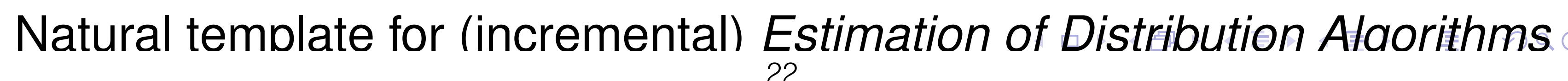




\section{The CMA-ES}

Input: $m \in \mathbb{R}^{n} ; \sigma \in \mathbb{R}_{+} ; \lambda \in \mathbb{N}_{\geq 2}$, usually $\lambda \geq 5$, default $4+\lfloor 3 \log n\rfloor$

Set $c_{m}=1 ; c_{1} \approx 2 / n^{2} ; c_{\mu} \approx \mu_{w} / n^{2} ; c_{\mathrm{c}} \approx 4 / n ; c_{\sigma} \approx 1 / \sqrt{n} ; d_{\sigma} \approx 1 ; w_{i=1 \ldots \lambda}$ decreasing in $i$ and $\sum_{i}^{\mu} w_{i}=1, w_{\mu}>0 \geq w_{\mu+1}, \mu_{w}^{-1}:=\sum_{i=1}^{\mu} w_{i}^{2} \approx 3 / \lambda$

Initialize $\mathrm{C}=\mathbf{I}$, and $p_{\mathrm{C}}=\mathbf{0}, p_{\sigma}=\mathbf{0}$

While not terminate

$$
\begin{array}{lrr}
\boldsymbol{x}_{i}=m+\sigma \boldsymbol{y}_{i}, \quad \text { where } \boldsymbol{y}_{i} \sim \mathcal{N}_{i}(\mathbf{0}, \mathrm{C}) \text { for } i=1, \ldots, \lambda & \text { sampling } \\
m \leftarrow m+c_{m} \sigma \boldsymbol{y}_{w}, \text { where } \boldsymbol{y}_{w}=\sum_{i=1}^{\mu} w_{\operatorname{rk}(i)} \boldsymbol{y}_{i} & \text { update mean } \\
p_{\sigma} \leftarrow\left(1-c_{\sigma}\right) p_{\sigma}+\sqrt{1-\left(1-c_{\sigma}\right)^{2}} \sqrt{\mu_{w}} \mathrm{C}^{-\frac{1}{2}} \boldsymbol{y}_{w} & \text { path for } \sigma \\
p_{\mathrm{C}} \leftarrow\left(1-c_{\mathrm{C}}\right) p_{\mathrm{C}}+\mathbb{1}_{[0,2 n]}\left\{\left\|p_{\sigma}\right\|^{2}\right\} \sqrt{1-\left(1-c_{\mathrm{C}}\right)^{2}} \sqrt{\mu_{w}} \boldsymbol{y}_{w} & \text { path for } \mathrm{C} \\
\sigma \leftarrow \sigma \times \exp \left(\frac{c_{\sigma}}{d_{\sigma}}\left(\frac{\left\|p_{\sigma}\right\|}{\mathrm{E}\|\mathcal{N}(\mathbf{0}, \mathbf{I})\|}-1\right)\right) & \text { update of } \sigma \\
\mathrm{C} \leftarrow \mathbf{C}+c_{\mu} \sum_{i=1}^{\lambda} w_{\operatorname{rk}(i)}\left(\boldsymbol{y}_{i} \boldsymbol{y}_{i}^{\top}-\mathrm{C}\right)+c_{1}\left(p_{\mathrm{C}} p_{\mathrm{C}}^{\top}-\mathrm{C}\right) & \text { update C }
\end{array}
$$

Not covered: termination, restarts, useful output, search boundaries and encoding, corrections for: positive definiteness guaranty, $p_{\mathrm{c}}$ variance loss, $c_{\sigma}$ and $d_{\sigma}$ for large $\lambda$ 


\section{Evolution Strategies}

New search points are sampled normally distributed

$$
\boldsymbol{x}_{i} \sim m+\sigma \mathcal{N}_{i}(\mathbf{0}, \mathbf{C}) \quad \text { for } i=1, \ldots, \lambda
$$

as perturbations of $m, \quad$ where $\boldsymbol{x}_{i}, m \in \mathbb{R}^{n}, \sigma \in \mathbb{R}_{+}, \mathrm{C} \in \mathbb{R}^{n \times n}$ where

- the mean vector $m \in \mathbb{R}^{n}$ represents the favorite solution

- the so-called step-size $\sigma \in \mathbb{R}_{+}$controls the step length

- the covariance matrix $\mathbf{C} \in \mathbb{R}^{n \times n}$ determines the shape of the distribution ellipsoid

here, all new points are sampled with the same parameters 


\section{Why Normal Distributions?}

(1) widely observed in nature, for example as phenotypic traits

(2) only stable distribution with finite variance

stable means that the sum of normal variates is again normal:

$$
\mathcal{N}(\boldsymbol{x}, \mathbf{A})+\mathcal{N}(\boldsymbol{y}, \mathbf{B}) \sim \mathcal{N}(\boldsymbol{x}+\boldsymbol{y}, \mathbf{A}+\mathbf{B})
$$

helpful in design and analysis of algorithms related to the central limit theorem

(3) most convenient way to generate isotropic search points the isotropic distribution does not favor any direction, rotational invariant

(4) maximum entropy distribution with finite variance the least possible assumptions on $f$ in the distribution shape 


\section{Normal Distribution}

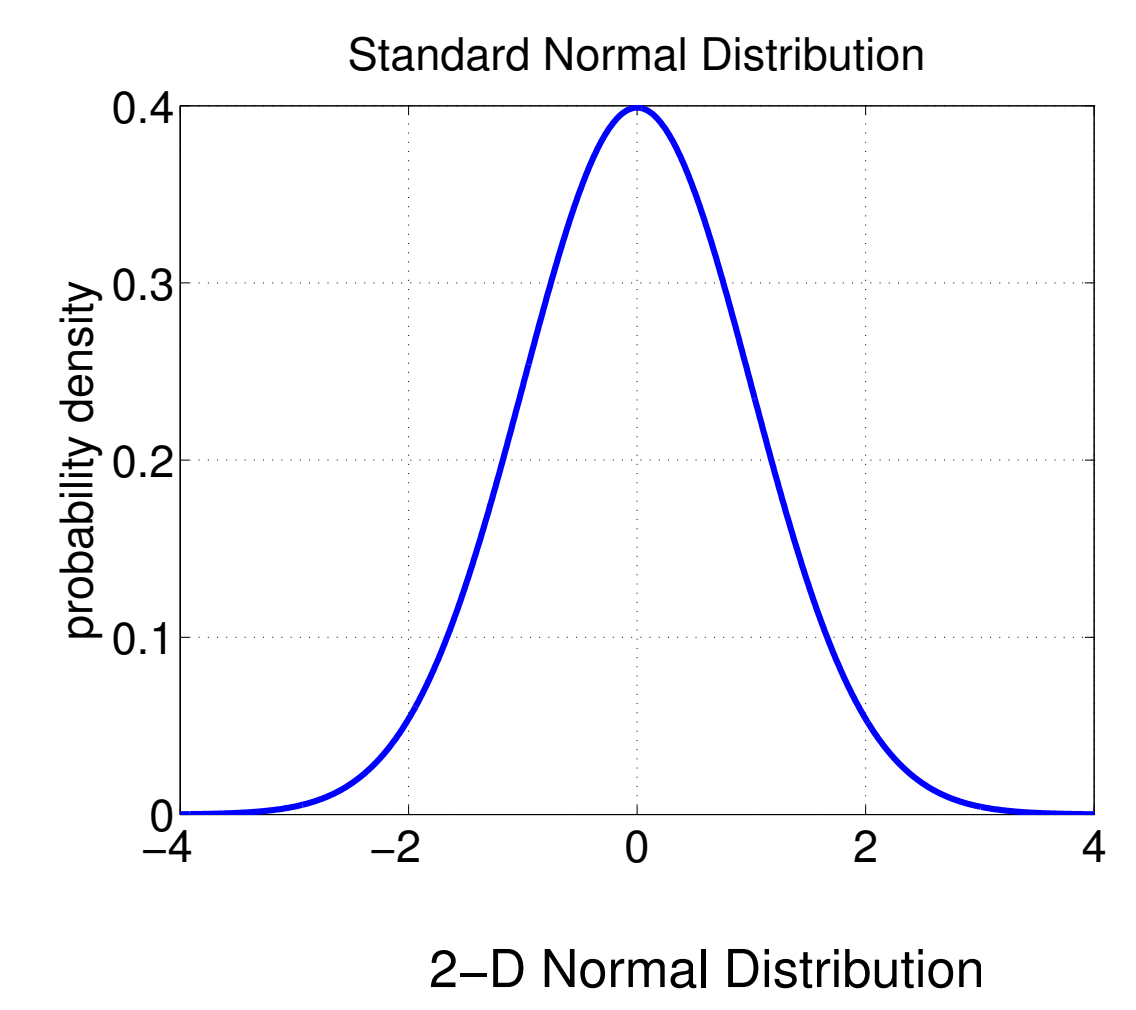

probability density of the 1-D standard normal distribution

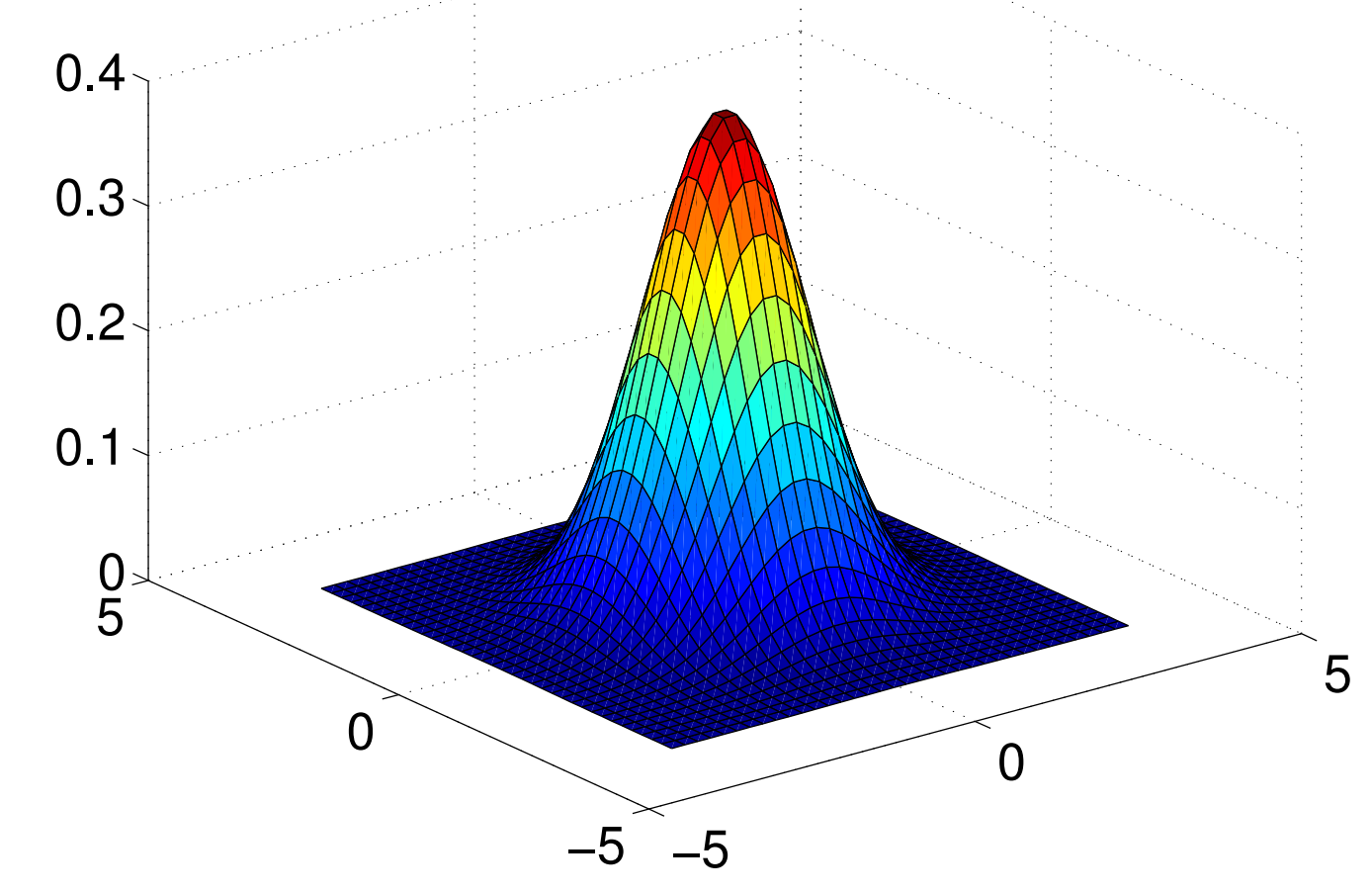

probability density of a 2-D normal distribution

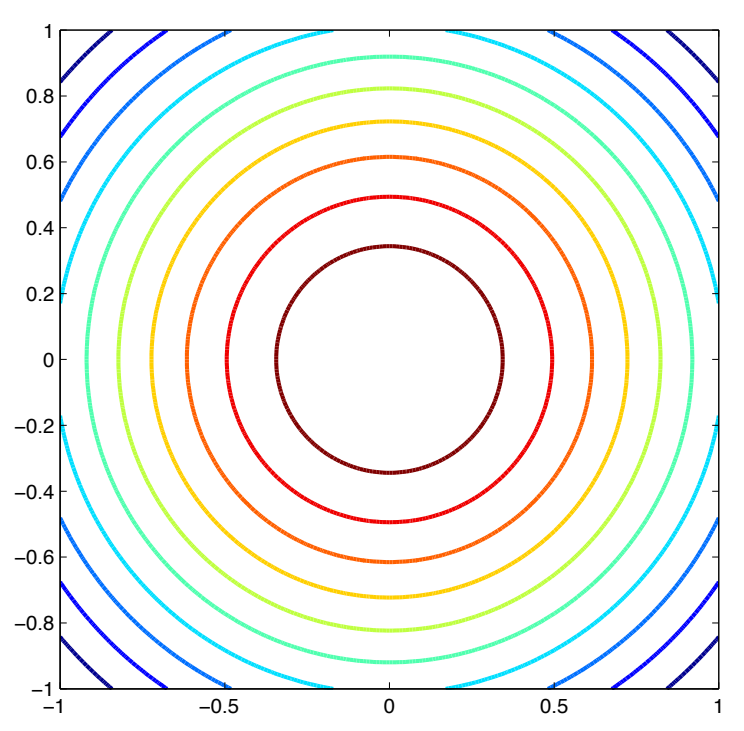




\section{The Multi-Variate ( $n$-Dimensional) Normal Distribution}

Any multi-variate normal distribution $\mathcal{N}(m, \mathrm{C})$ is uniquely determined by its mean value $m \in \mathbb{R}^{n}$ and its symmetric positive definite $n \times n$ covariance matrix $\mathrm{C}$.

The mean value $m$

- determines the displacement (translation)

- value with the largest density (modal value)

- the distribution is symmetric about the distribution mean

2-D Normal Distribution

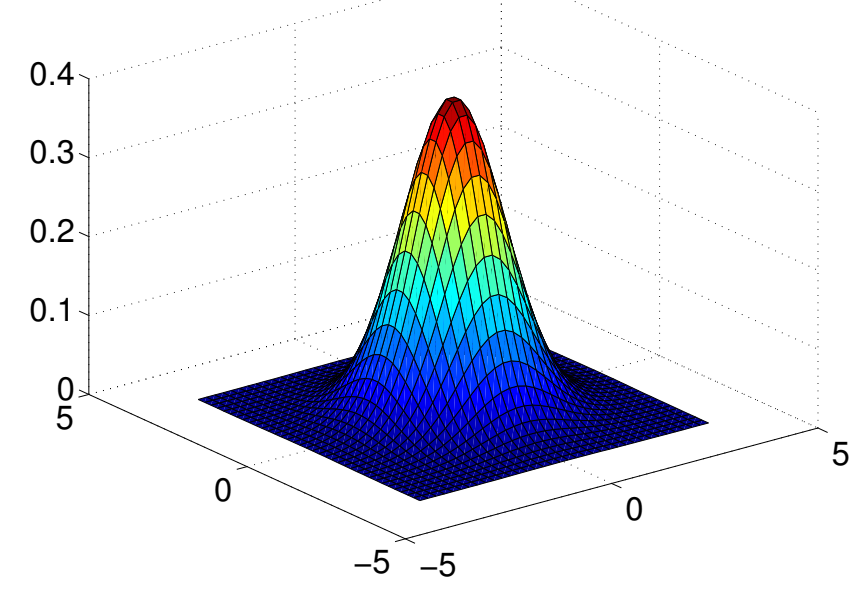


... any covariance matrix can be uniquely identified with the iso-density ellipsoid $\left\{\boldsymbol{x} \in \mathbb{R}^{n} \mid(\boldsymbol{x}-m)^{\mathrm{T}} \mathbf{C}^{-1}(\boldsymbol{x}-m)=n\right\}$

Lines of Equal Density

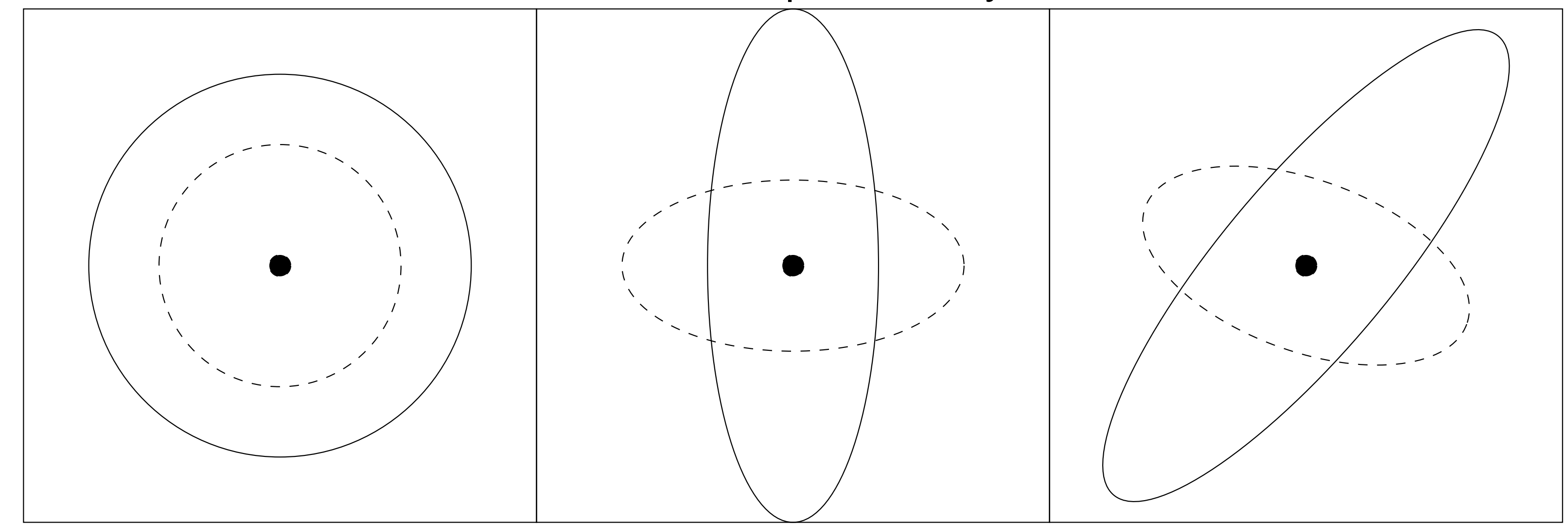

$$
\mathcal{N}\left(m, \sigma^{2} \mathbf{I}\right) \sim m+\sigma \mathcal{N}(\mathbf{0}, \mathbf{I})
$$

one degree of freedom $\sigma$

components are

independent standard

normally distributed

where $\mathbf{I}$ is the identity matrix (isotropic case) and $\mathbf{D}$ is a diagonal matrix (reasonable for separable problems) and $\mathbf{A} \times \mathcal{N}(\mathbf{0}, \mathbf{I}) \sim \mathcal{N}\left(\mathbf{0}, \mathbf{A} \mathbf{A}^{\mathrm{T}}\right)$ holds for all $\mathbf{A}$. 


\section{Multivariate Normal Distribution and Eigenvalues}

For any positive definite symmetric $\mathbf{C}$,

$$
\mathbf{C}=d_{1}^{2} \boldsymbol{b}_{1} \boldsymbol{b}_{1}^{\mathrm{T}}+\cdots+d_{N}^{2} \boldsymbol{b}_{N} \boldsymbol{b}_{N}^{\mathrm{T}}
$$

$d_{i}$ : square root of the eigenvalue of $\mathbf{C}$

$\boldsymbol{b}_{i}$ : eigenvector of $\mathbf{C}$, corresponding to $d_{i}$

The multivariate normal distribution $\mathcal{N}(\boldsymbol{m}, \mathbf{C})$

$$
\mathcal{N}(\boldsymbol{m}, \mathbf{C}) \sim \boldsymbol{m}+\mathcal{N}\left(0, d_{1}^{2}\right) \boldsymbol{b}_{1}+\cdots+\mathcal{N}\left(0, d_{N}^{2}\right) \boldsymbol{b}_{N}
$$

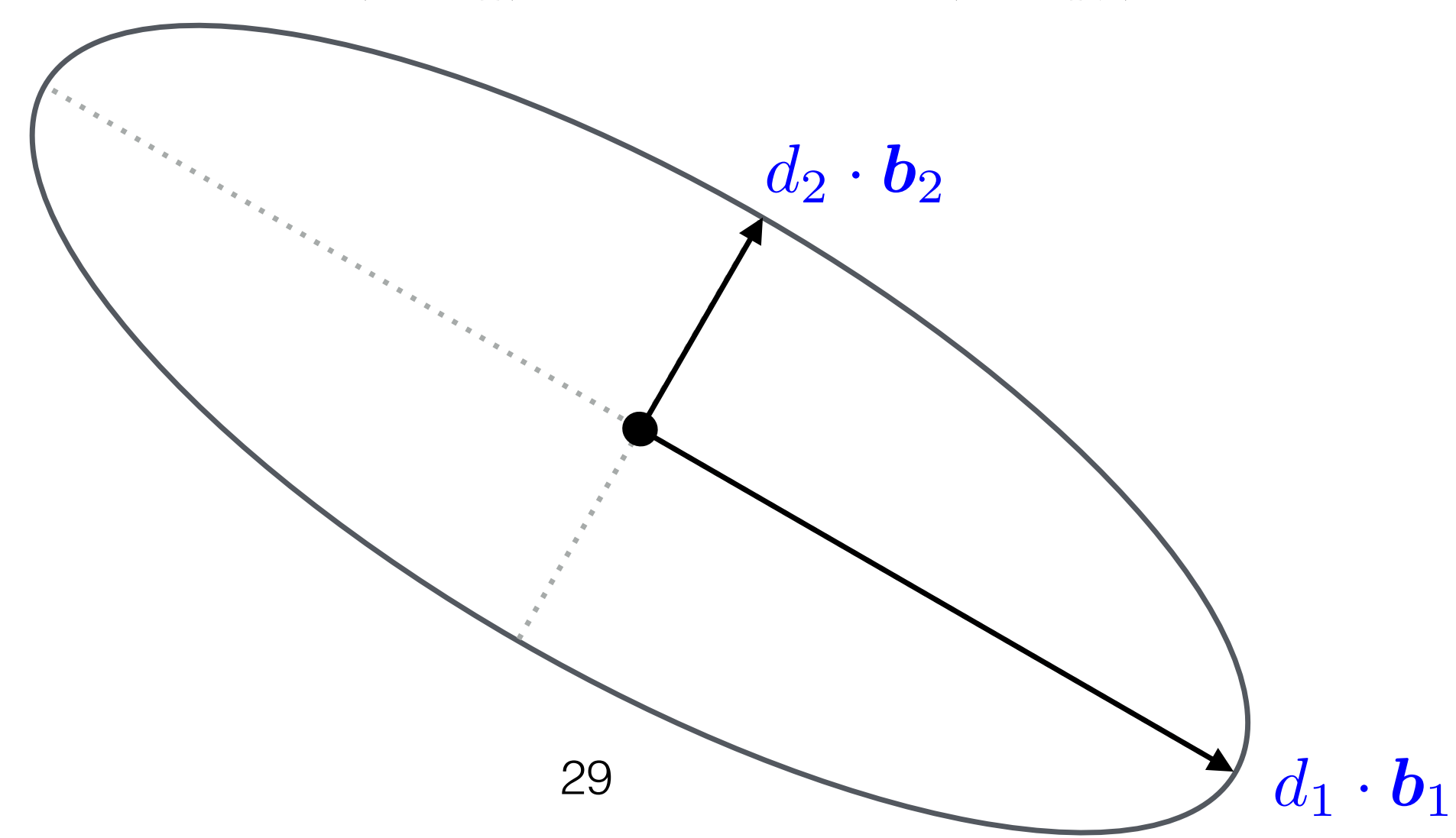




\section{The $(\mu / \mu, \lambda)$-ES}

Non-elitist selection and intermediate (weighted) recombination Given the $i$-th solution point $\boldsymbol{x}_{i}=\boldsymbol{m}+\sigma \underbrace{\mathcal{N}_{i}(\mathbf{0}, \mathbf{C})}=m+\sigma \boldsymbol{y}_{i}$ $=: y_{i}$

Let $\boldsymbol{x}_{i: \lambda}$ the $i$-th ranked solution point, such that $f\left(\boldsymbol{x}_{1: \lambda}\right) \leq \cdots \leq f\left(\boldsymbol{x}_{\lambda: \lambda}\right)$.

The best $\mu$ points are selected from the new solutions (non-elitistic) and weighted intermediate recombination is applied. 


\section{Invariance Under Monotonically Increasing Functions}

\section{Rank-based algorithms}

Update of all parameters uses only the ranks

$$
f\left(x_{1: \lambda}\right) \leq f\left(x_{2: \lambda}\right) \leq \ldots \leq f\left(x_{\lambda: \lambda}\right)
$$

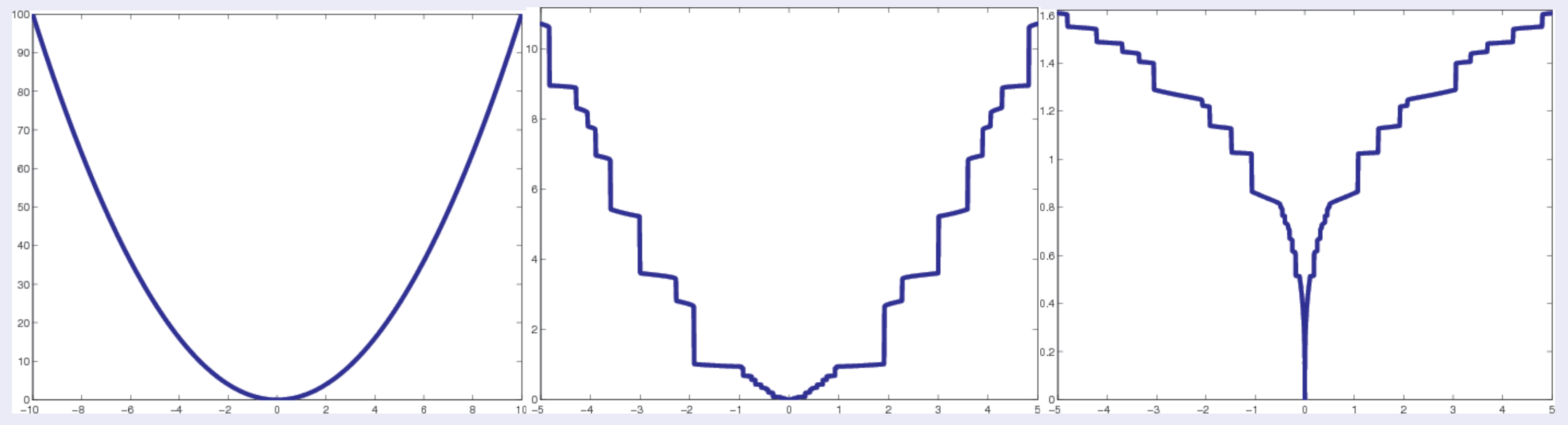

$$
g\left(f\left(x_{1: \lambda}\right)\right) \leq g\left(f\left(x_{2: \lambda}\right)\right) \leq \ldots \leq g\left(f\left(x_{\lambda: \lambda}\right)\right) \quad \forall g
$$

$g$ is strictly monotonically increasing 


\section{Basic Invariance in Search Space}

- translation invariance

is true for most optimization algorithms

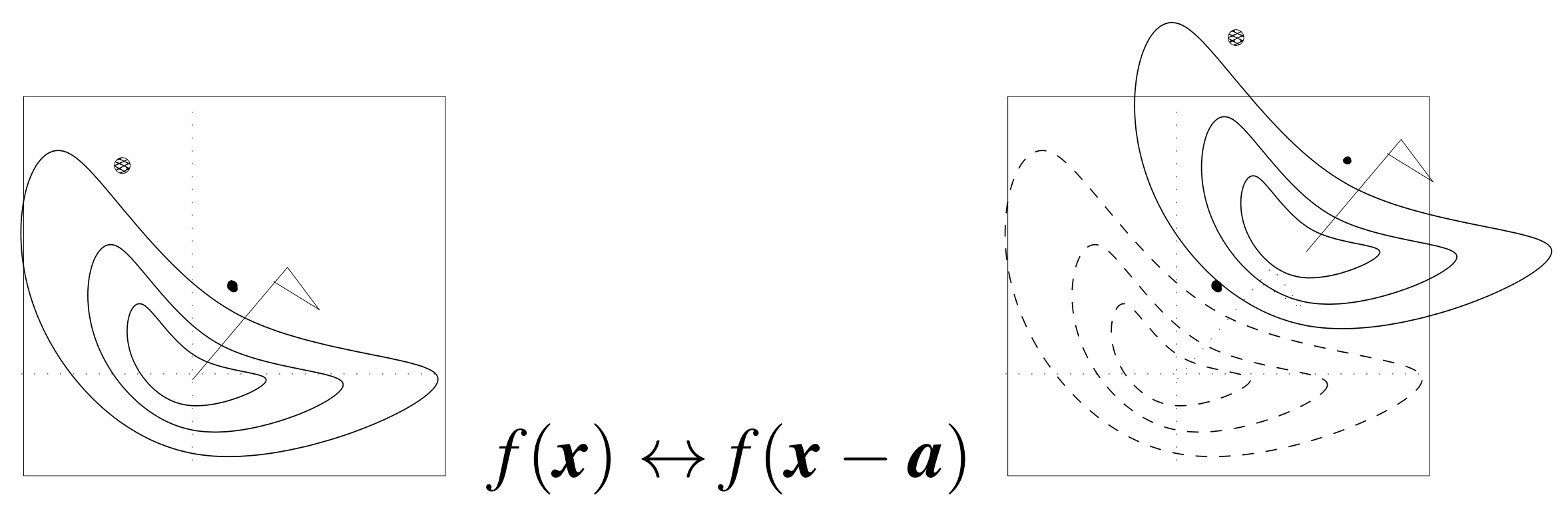

Identical behavior on $f$ and $f_{a}$

$$
\begin{aligned}
f: & \boldsymbol{x} \mapsto f(\boldsymbol{x}), & & \boldsymbol{x}^{(t=0)}=\boldsymbol{x}_{0} \\
f_{\boldsymbol{a}}: & \boldsymbol{x} \mapsto f(\boldsymbol{x}-\boldsymbol{a}), & & \boldsymbol{x}^{(t=0)}=\boldsymbol{x}_{0}+\boldsymbol{a}
\end{aligned}
$$

No difference can be observed w.r.t. the argument of $f$ 


\section{Summary}

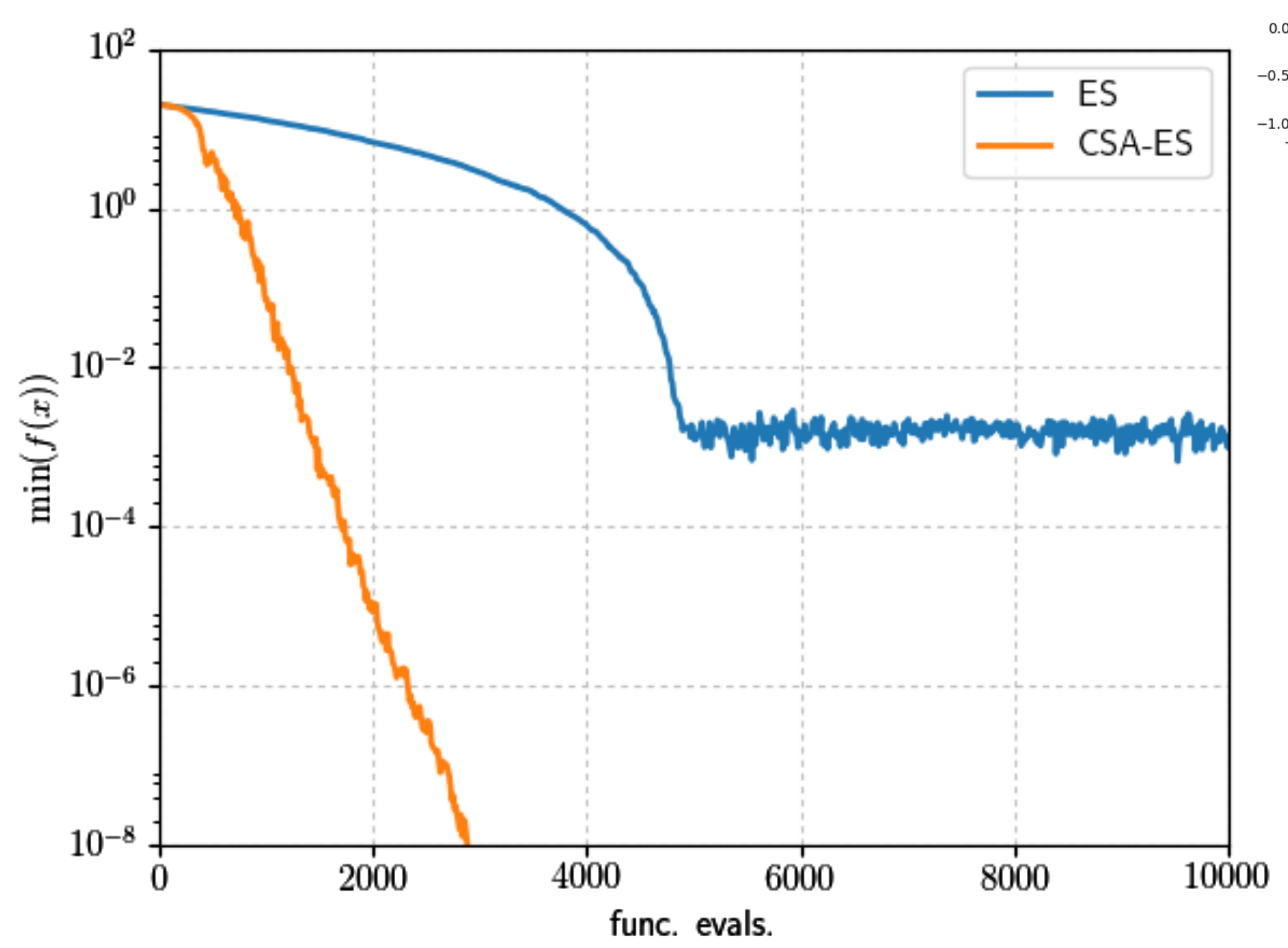

On 20D Sphere Function: $f(\mathbf{x})=\sum_{i=1}^{N}[\mathbf{x}]_{i}^{2}$

- ES without adaptation can't approach the optimum $\Rightarrow$ adaptation required 


\section{Evolution Strategies}

Recalling

New search points are sampled normally distributed

$$
\boldsymbol{x}_{i} \sim m+\sigma \mathcal{N}_{i}(\mathbf{0}, \mathrm{C}) \quad \text { for } i=1, \ldots, \lambda
$$

as perturbations of $m, \quad$ where $\boldsymbol{x}_{i}, m \in \mathbb{R}^{n}, \sigma \in \mathbb{R}_{+}, \mathbf{C} \in \mathbb{R}^{n \times n}$

where

- the mean vector $m \in \mathbb{R}^{n}$ represents the favorite solution and $m \leftarrow \sum_{i=1}^{\mu} w_{i} \boldsymbol{x}_{i: \lambda}$

- the so-called step-size $\sigma \in \mathbb{R}_{+}$controls the step length

- the covariance matrix $\mathrm{C} \in \mathbb{R}^{n \times n}$ determines the shape of the distribution ellipsoid

The remaining question is how to update $\sigma$ and $\mathbf{C}$. 


\section{Methods for Step-Size Control}

- 1/5-th success rule ${ }^{a b}$, often applied with "+"-selection

increase step-size if more than $20 \%$ of the new solutions are successful, decrease otherwise

- $\sigma$-self-adaptationc, applied with ","-selection

mutation is applied to the step-size and the better, according to the objective function value, is selected

simplified "global" self-adaptation

- path length control ${ }^{d}$ (Cumulative Step-size Adaptation, CSA)

self-adaptation derandomized and non-localized

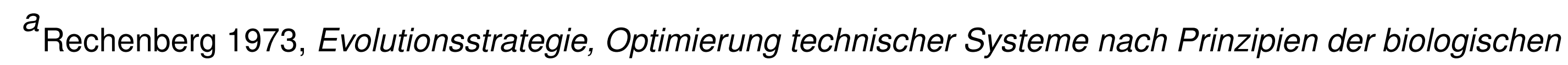
Evolution, Frommann-Holzboog

${ }^{b}$ Schumer and Steiglitz 1968. Adaptive step size random search. IEEE TAC

${ }^{c}$ Schwefel 1981, Numerical Optimization of Computer Models, Wiley

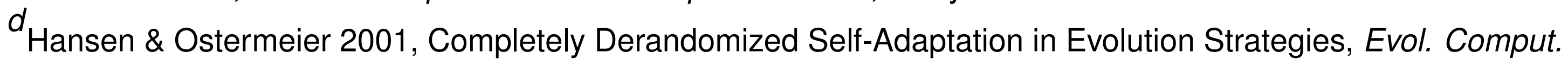
9(2)

${ }^{e}$ Ostermeier et al 1994, Step-size adaptation based on non-local use of selection information, PPSN IV 


\section{Path Length Control (CSA)}

The Concept of Cumulative Step-Size Adaptation

$$
\begin{aligned}
& \boldsymbol{x}_{i}=m+\sigma \boldsymbol{y}_{i} \\
& m \leftarrow m+\sigma \boldsymbol{y}_{w}
\end{aligned}
$$

\section{Measure the length of the evolution path}

the pathway of the mean vector $m$ in the generation sequence

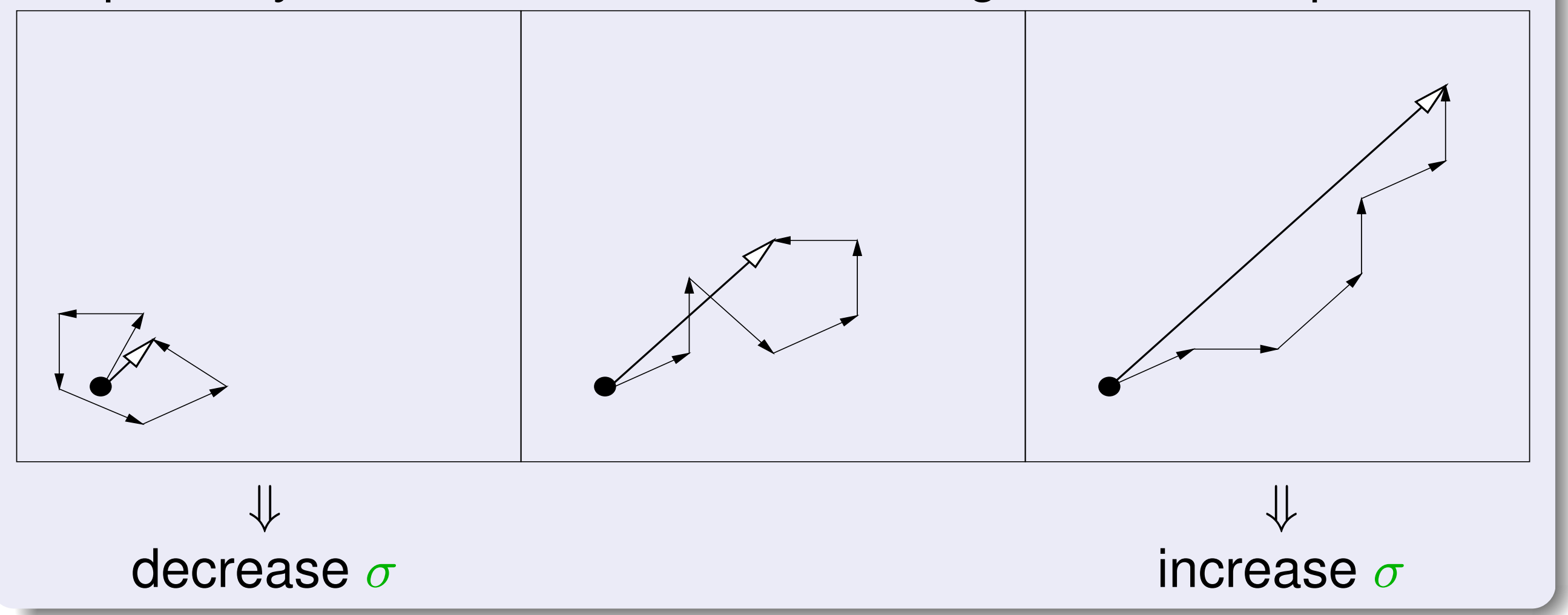

loosely speaking steps are

- perpendicular under random selection (in expectation)

- perpendicular in the desired situation (to be most efficient) 


\section{Path Length Control (CSA)}

The Equations

Initialize $m \in \mathbb{R}^{n}, \sigma \in \mathbb{R}_{+}$, evolution path $p_{\sigma}=\mathbf{0}$, set $c_{\sigma} \approx 4 / n, d_{\sigma} \approx 1$. 


\section{$(5 / 5,10)$-CSA-ES, default parameters}

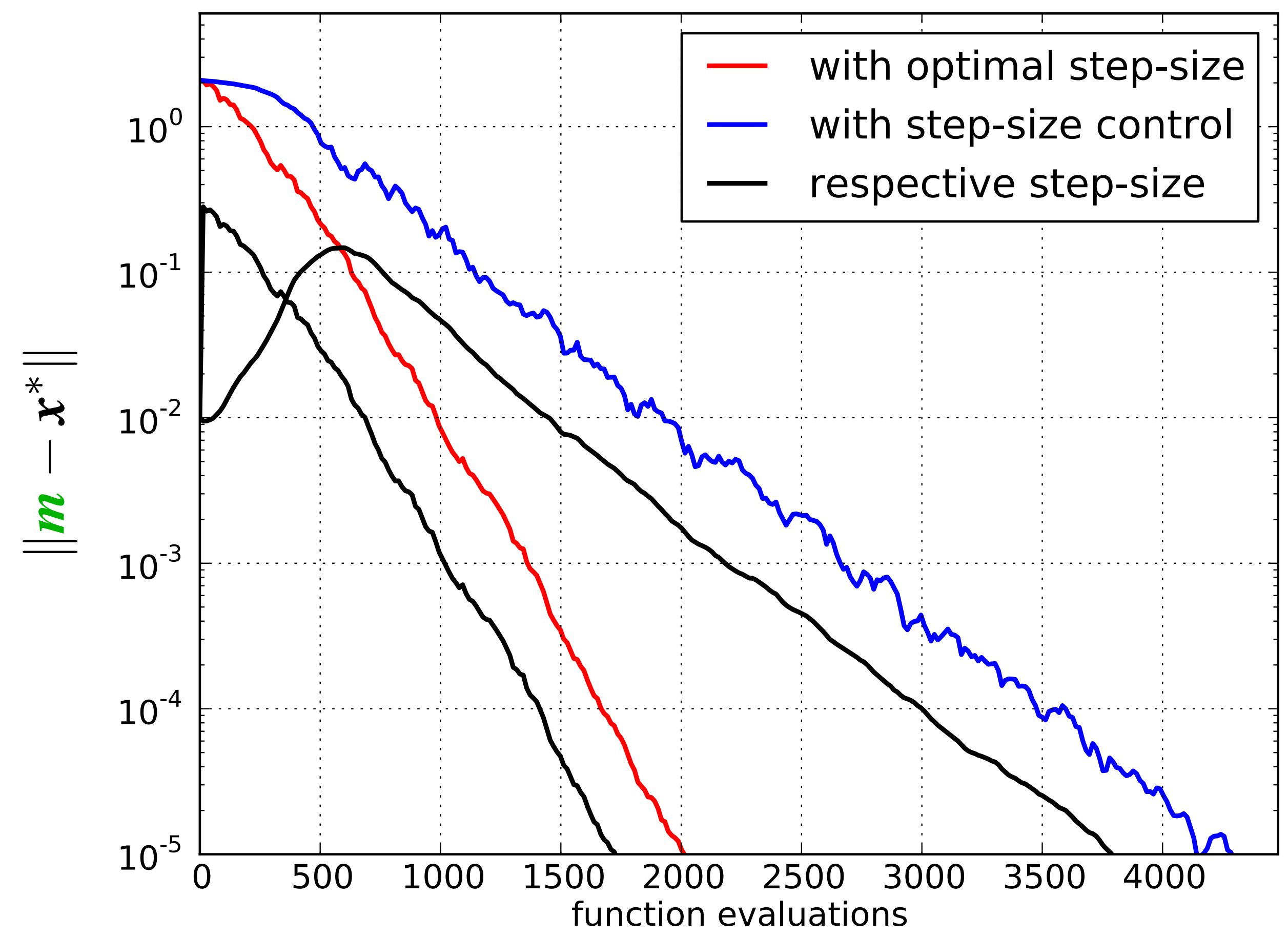

$$
f(\boldsymbol{x})=\sum_{i=1}^{n} x_{i}^{2}
$$$$
\text { in }[-0.2,0.8]^{n}
$$$$
\text { for } n=30
$$ 


\section{Two-Point Step-Size Adaptation (TPA)}

- Sample a pair of symmetric points along the previous mean shift

$$
\boldsymbol{x}_{1 / 2}=m^{(g)} \pm \sigma^{(g)} \frac{\|\mathcal{N}(\mathbf{0}, \mathbf{I})\|}{\left\|m^{(g)}-m^{(g-1)}\right\|_{\mathbf{C}^{(g)}}}\left(m^{(g)}-m^{(g-1)}\right) \quad\|\boldsymbol{x}\|_{\mathbf{C}}:=\boldsymbol{x}^{\mathrm{T}} \mathbf{C}^{-1} \boldsymbol{x}
$$

- Compare the ranking of $x_{1}$ and $x_{2}$ among $\lambda$ current populations

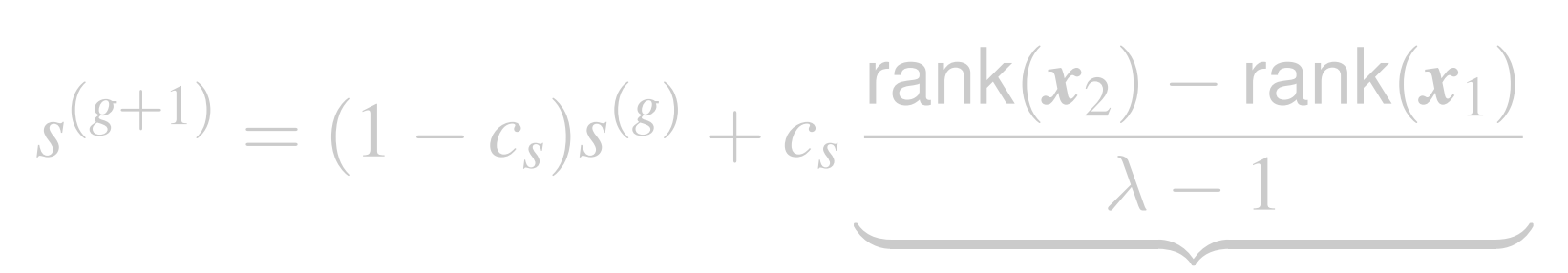

$>0$ if the previous step still produces a promising solution

- Update the step-size

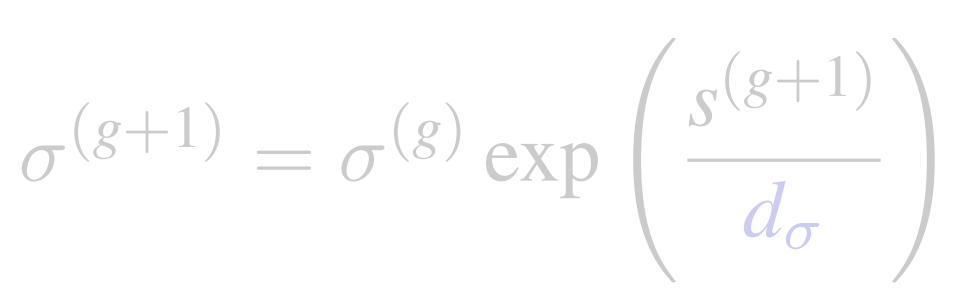

[Hansen, 2008] Hansen, N. (2008). CMA-ES with two-point step-size adaptation. [research report] rr-6527, 2008 . Inria-00276854v5. [Hansen et al., 2014] Hansen, N., Atamna, A., and Auger, A. (2014). How to assess step-size ada,jtation mechanisms in randomised search. In Parallel Problem Solving from Nature-PPSN XIII, pages 60-69. Springer. 


\section{Alternatives: Success-Based Step-Size Control}

comparing the fitness distributions of current and previous iterations

Generalizations of 1/5th-success-rule for non-elitist and multi-recombinant ES

- Median Success Rule [Ait Elhara et al., 2013]

- Population Success Rule [Loshchilov, 2014]

controls a success probability

An advantage over CSA and TPA: Cheap Computation

- It depends only on $\lambda$.

- cf. CSA and TPA require a computation of $C^{-1 / 2} x$ and $C^{-1} x$, respectively.

[Ait Elhara et al., 2013] Ait Elhara, O., Auger, A., and Hansen, N. (2013). A median success rule for non- elitist evolution strategies: Study of feasibility. In Proc. of the GECCO, pages 415-422.

[Loshchilov, 2014] Loshchilov, I. (2014). A computationally efficient limited memory cma-es for large scale optimization. In Proc. of the GECCO, pages 397-404. 


\section{Step-Size Control: Summary}

Why Step-Size Control?

- to achieve linear convergence at near-optimal rate

Cumulative Step-Size Adaptation

- efficient and robust for $\lambda \leq N$

- inefficient on functions with (many) ineffective axes

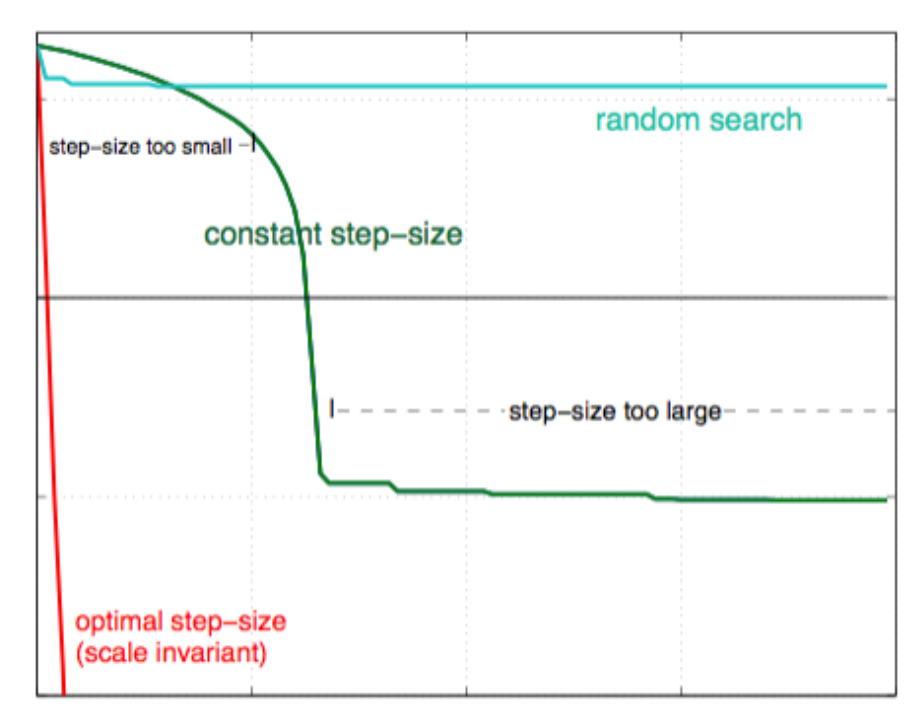

Alternative Step-Size Adaptation Mechanisms

- Two-Point Step-Size Adaptation

- Median Success Rule, Population Success Rule

the effective adaptation of the overall population diversity seems yet to pose open questions, in particular with recombination or without entire control over the realized distribution. ${ }^{a}$

${ }^{a}$ Hansen et al. How to Assess Step-Size Adaptation Mechanisms in Randomised Search. PPSN 2014 


\section{Step-Size Control: Summary}

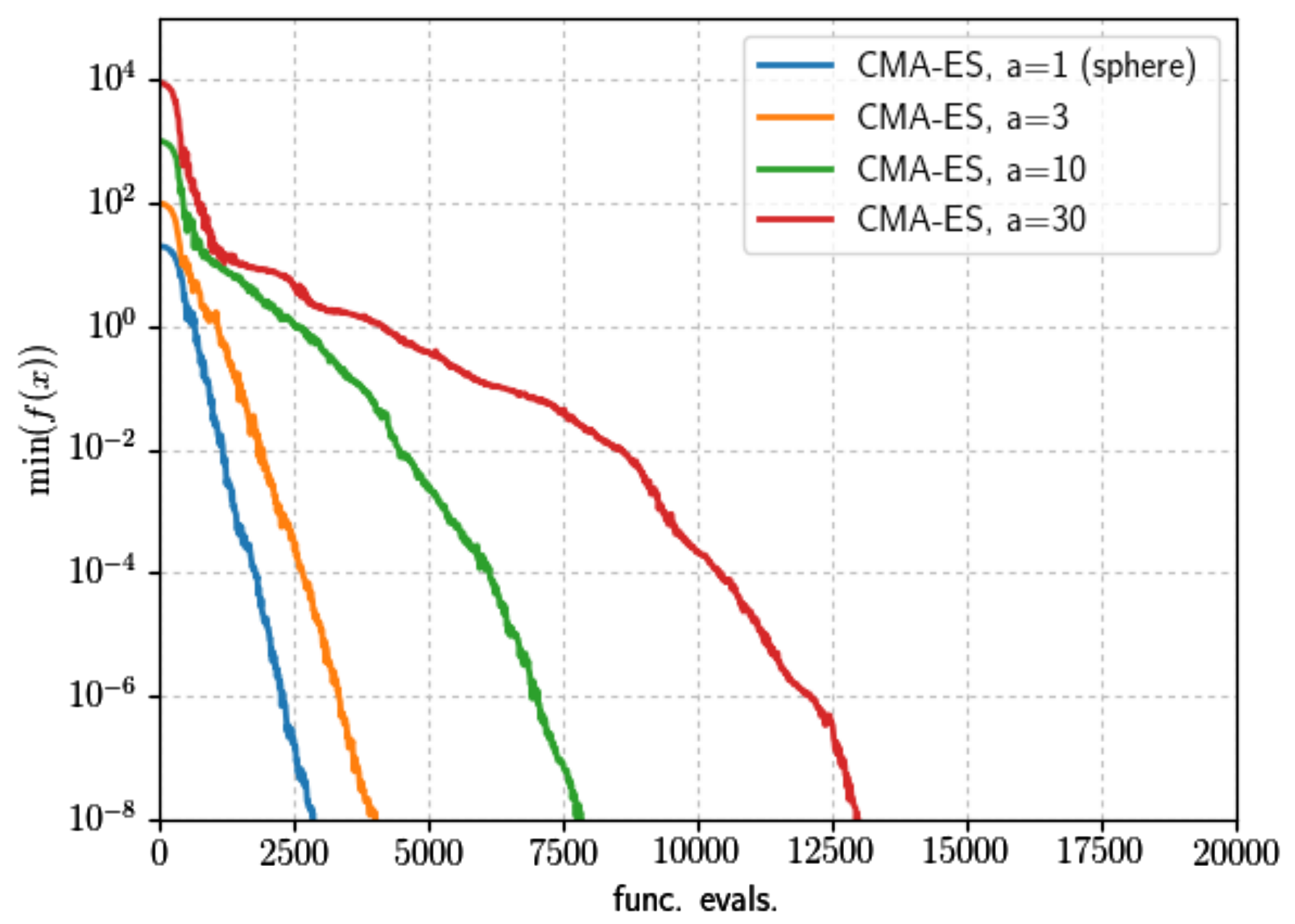

On 20D TwoAxes Function: $f(\mathbf{x})=\sum_{i=1}^{N / 2}[\mathbf{R x}]_{i}^{2}+a^{2} \sum_{i=N / 2+1}^{N}[\mathbf{R x}]_{i}^{2}$, $\mathbf{R}$ : orthogonal

- convergence speed of CSA-ES becomes lower as the function becomes ill conditioned $\left(a^{2}\right.$ becomes greater $) \Rightarrow$ covariance matrix adaptation required

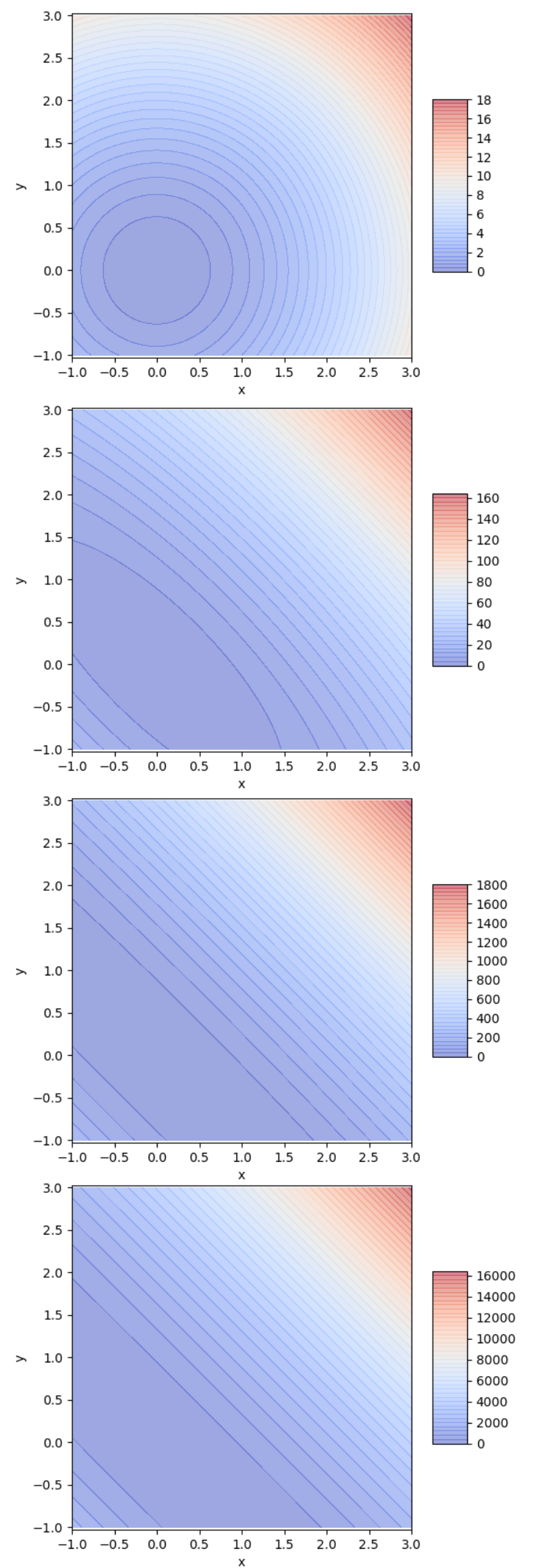




\section{Evolution Strategies}

Recalling

New search points are sampled normally distributed

$$
\boldsymbol{x}_{i} \sim m+\sigma \mathcal{N}_{i}(\mathbf{0}, \mathbf{C}) \quad \text { for } i=1, \ldots, \lambda
$$

as perturbations of $m, \quad$ where $\boldsymbol{x}_{i}, m \in \mathbb{R}^{n}, \sigma \in \mathbb{R}_{+}, \mathbf{C} \in \mathbb{R}^{n \times n}$ where

- the mean vector $m \in \mathbb{R}^{n}$ represents the favorite solution

- the so-called step-size $\sigma \in \mathbb{R}_{+}$controls the step length

- the covariance matrix $\mathbf{C} \in \mathbb{R}^{n \times n}$ determines the shape of the distribution ellipsoid

The remaining question is how to update $\mathrm{C}$. 


\section{Covariance Matrix Adaptation}

\section{Rank-One Update}

$$
m \leftarrow m+\sigma \boldsymbol{y}_{w}, \quad \boldsymbol{y}_{w}=\sum_{i=1}^{\mu} w_{i} \boldsymbol{y}_{i: \lambda}, \quad \boldsymbol{y}_{i} \sim \mathcal{N}_{i}(\mathbf{0}, \mathbf{C})
$$

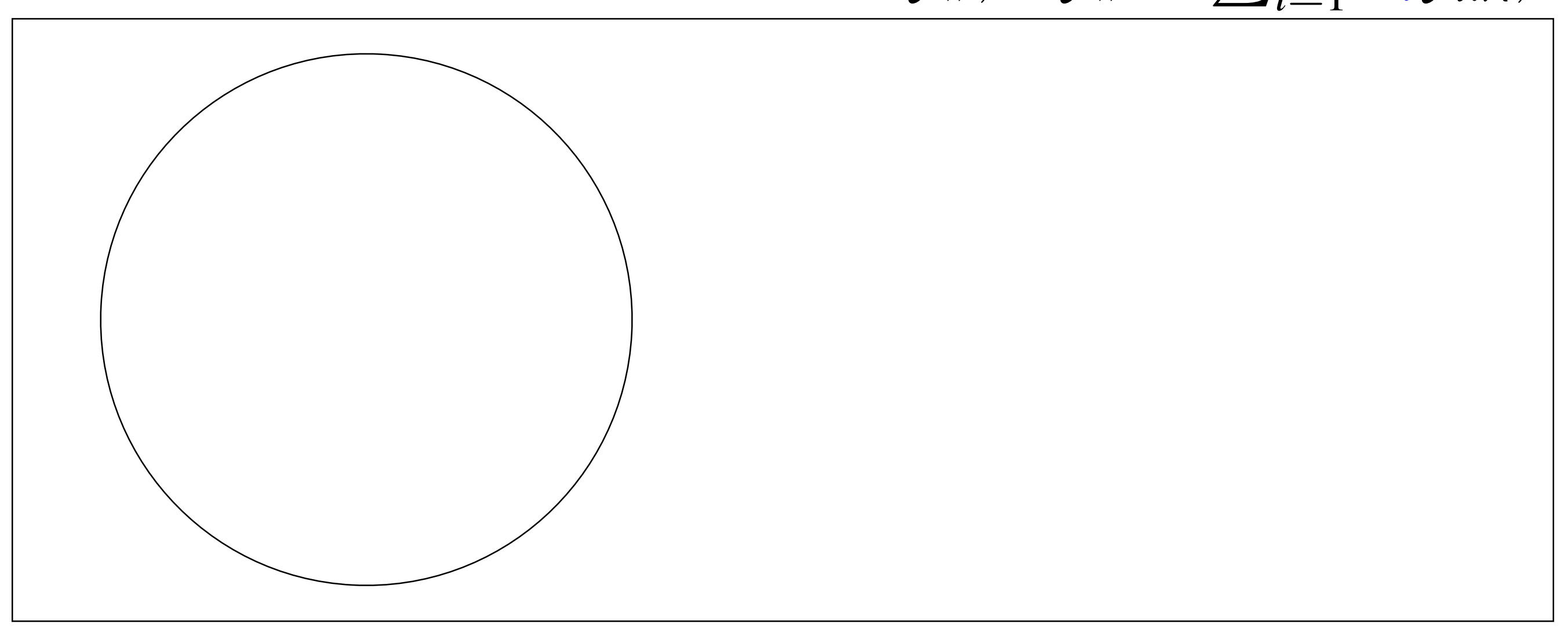

initial distribution, $\mathbf{C}=\mathbf{I}$ 


\section{Covariance Matrix Adaptation}

Rank-One Update

$$
m \leftarrow \boldsymbol{m}+\sigma \boldsymbol{y}_{w}, \quad \boldsymbol{y}_{w}=\sum_{i=1}^{\mu} w_{i} \boldsymbol{y}_{i: \lambda}, \quad \boldsymbol{y}_{i} \sim \mathcal{N}_{i}(\mathbf{0}, \mathbf{C})
$$

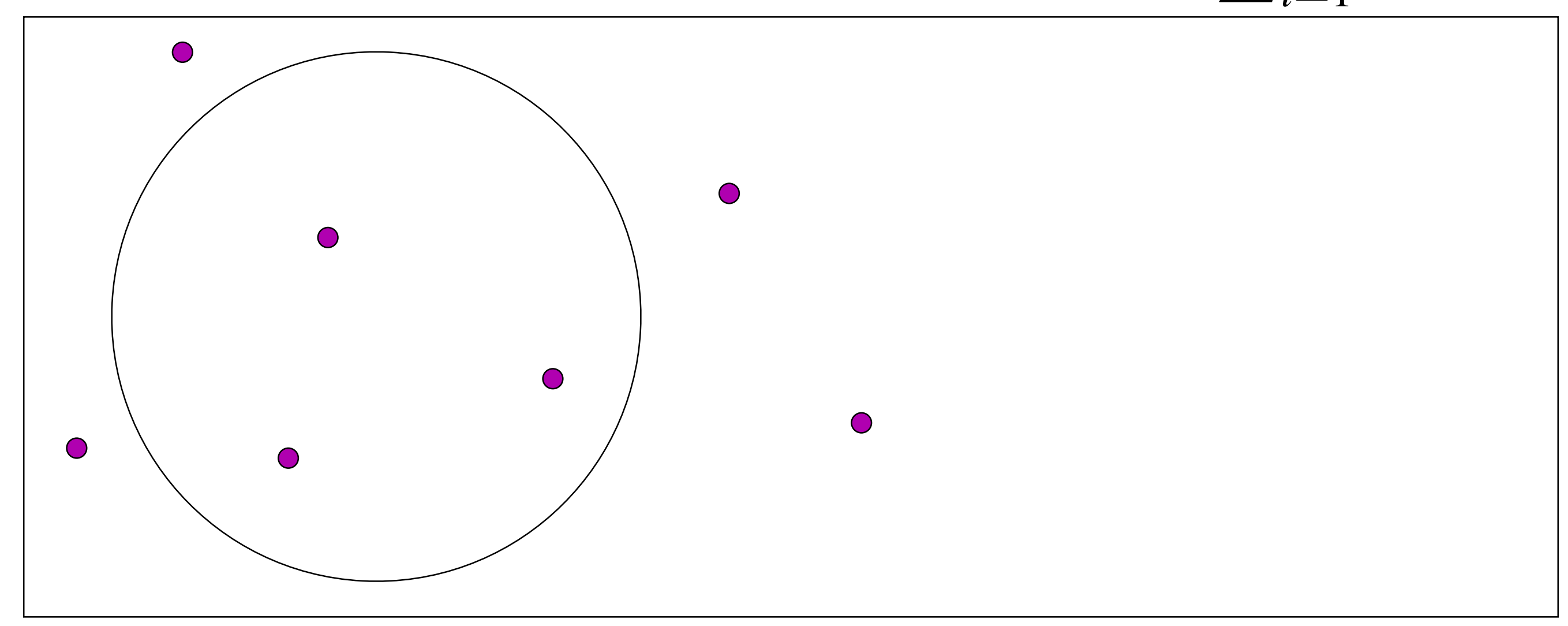

initial distribution, $\mathbf{C}=\mathbf{I}$ 


\section{Covariance Matrix Adaptation}

Rank-One Update

$$
m \leftarrow \boldsymbol{m}+\sigma \boldsymbol{y}_{w}, \quad \boldsymbol{y}_{w}=\sum_{i=1}^{\mu} w_{i} \boldsymbol{y}_{i: \lambda}, \quad \boldsymbol{y}_{i} \sim \mathcal{N}_{i}(\mathbf{0}, \mathbf{C})
$$

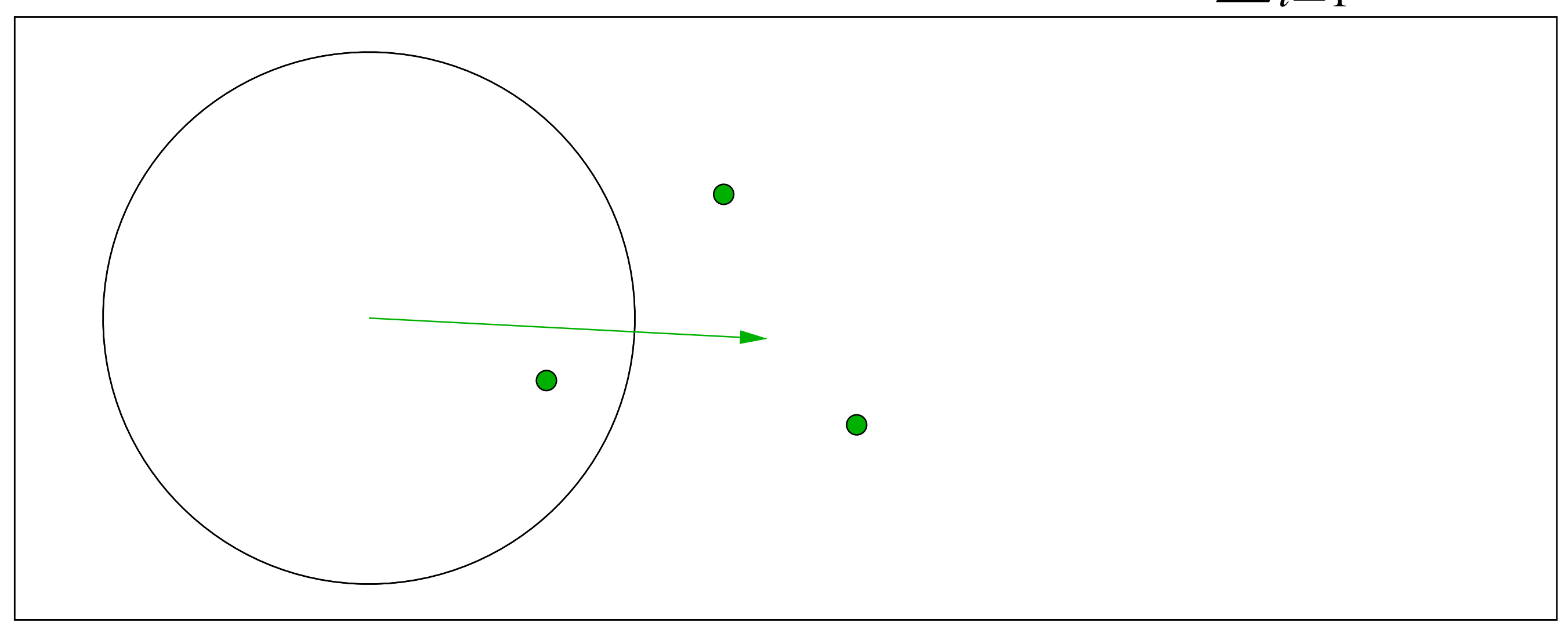

$\boldsymbol{y}_{w}$, movement of the population mean $m$ (disregarding $\sigma$ ) 


\section{Covariance Matrix Adaptation}

\section{Rank-One Update}

$$
m \leftarrow m+\sigma \boldsymbol{y}_{w}, \quad \boldsymbol{y}_{w}=\sum_{i=1}^{\mu} w_{i} \boldsymbol{y}_{i: \lambda}, \quad \boldsymbol{y}_{i} \sim \mathcal{N}_{i}(\mathbf{0}, \mathbf{C})
$$

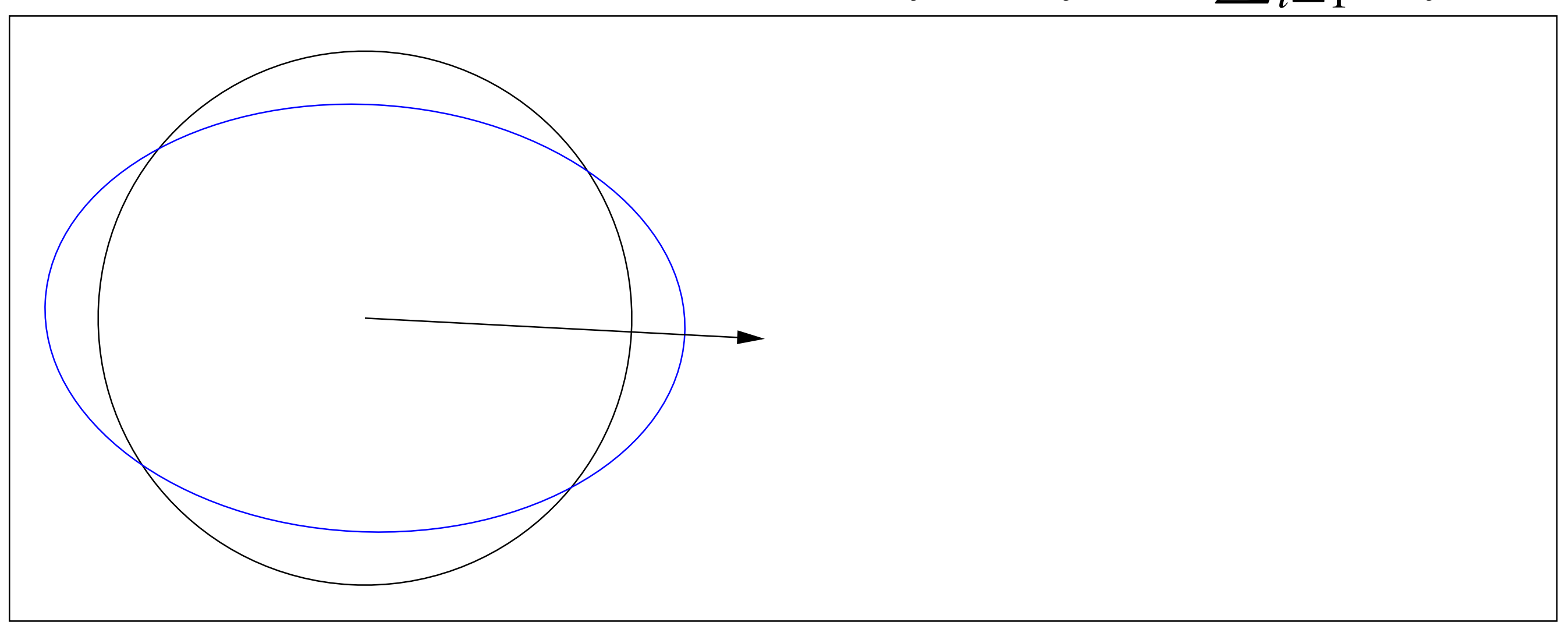

mixture of distribution $\mathbf{C}$ and step $\boldsymbol{y}_{w}$,

$\mathbf{C} \leftarrow 0.8 \times \mathbf{C}+0.2 \times \boldsymbol{y}_{w} \boldsymbol{y}_{w}^{\mathrm{T}}$ 


\section{Covariance Matrix Adaptation}

\section{Rank-One Update}

$$
m \leftarrow m+\sigma \boldsymbol{y}_{w}, \quad \boldsymbol{y}_{w}=\sum_{i=1}^{\mu} w_{i} \boldsymbol{y}_{i: \lambda}, \quad \boldsymbol{y}_{i} \sim \mathcal{N}_{i}(\mathbf{0}, \mathbf{C})
$$

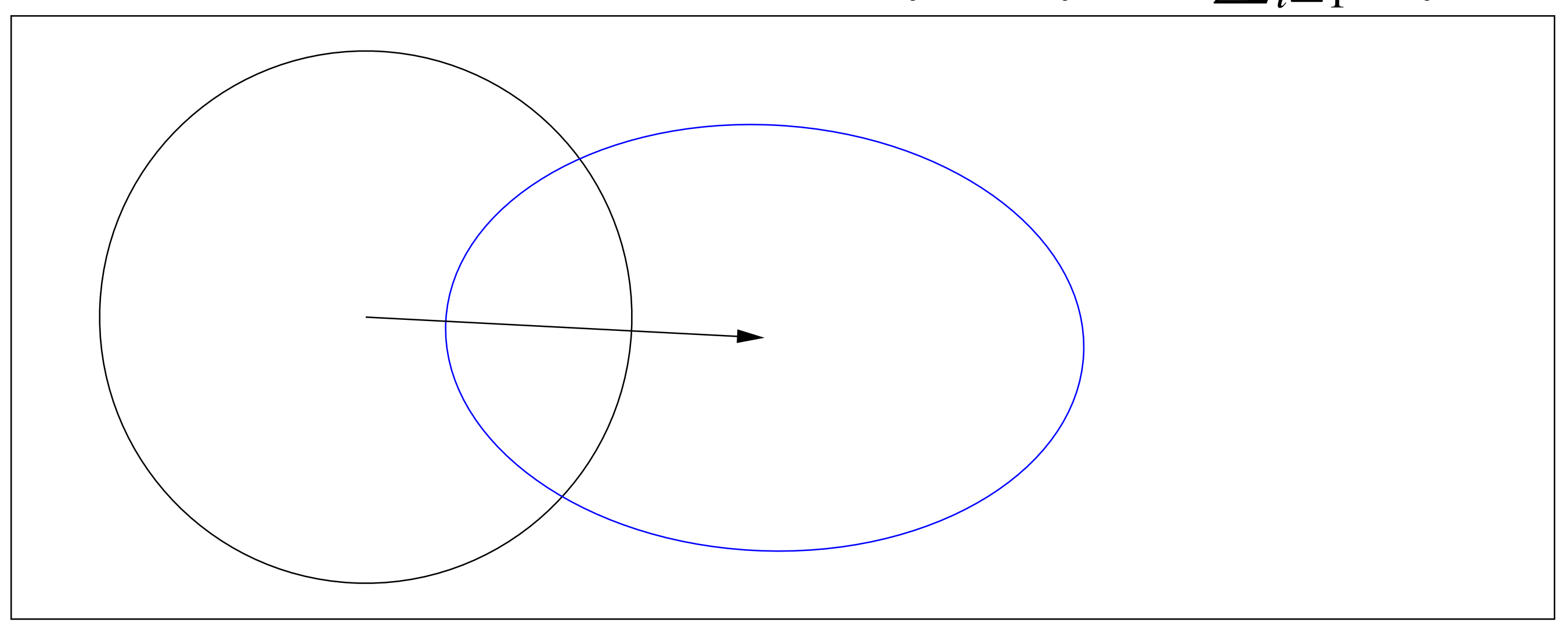

new distribution (disregarding $\sigma$ ) 


\section{Covariance Matrix Adaptation}

\section{Rank-One Update}

$$
m \leftarrow m+\sigma \boldsymbol{y}_{w}, \quad \boldsymbol{y}_{w}=\sum_{i=1}^{\mu} w_{i} \boldsymbol{y}_{i: \lambda}, \quad \boldsymbol{y}_{i} \sim \mathcal{N}_{i}(\mathbf{0}, \mathbf{C})
$$

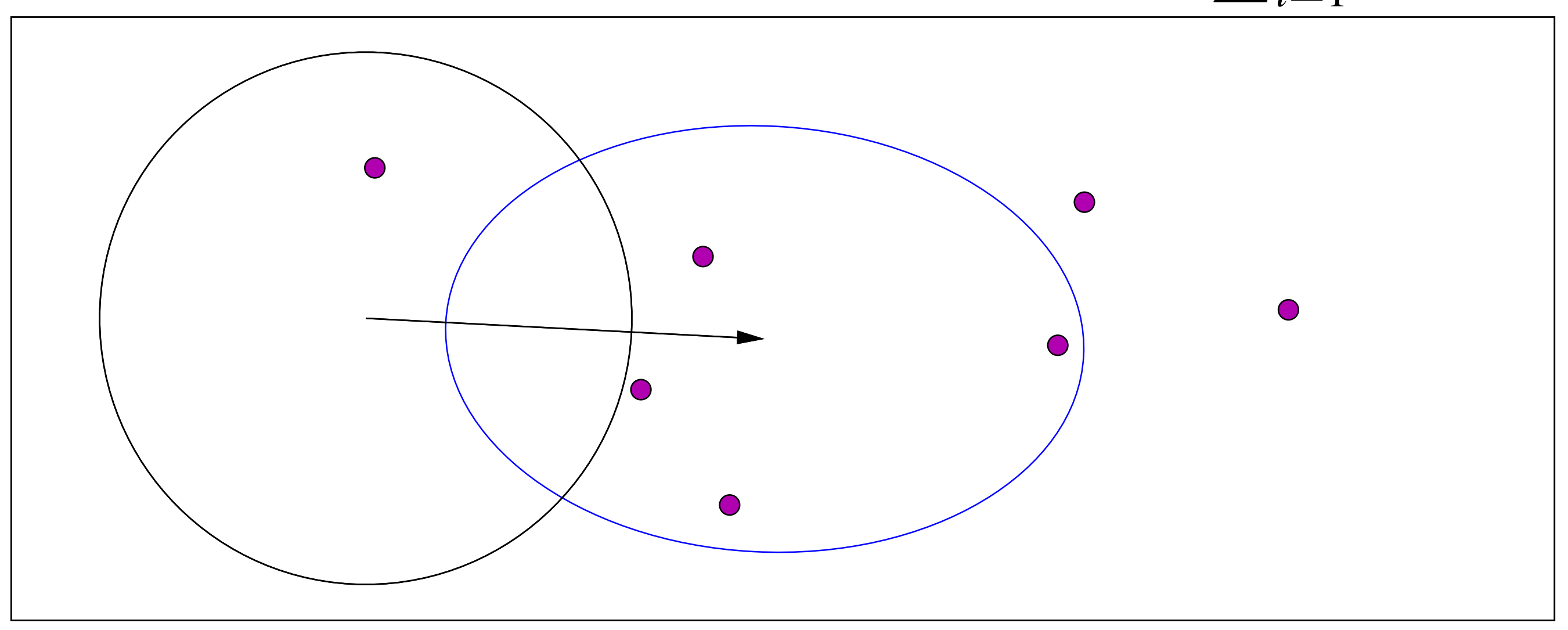

new distribution (disregarding $\sigma$ ) 


\section{Covariance Matrix Adaptation}

\section{Rank-One Update}

$$
m \leftarrow m+\sigma \boldsymbol{y}_{w}, \quad \boldsymbol{y}_{w}=\sum_{i=1}^{\mu} w_{i} \boldsymbol{y}_{i: \lambda}, \quad \boldsymbol{y}_{i} \sim \mathcal{N}_{i}(\mathbf{0}, \mathbf{C})
$$

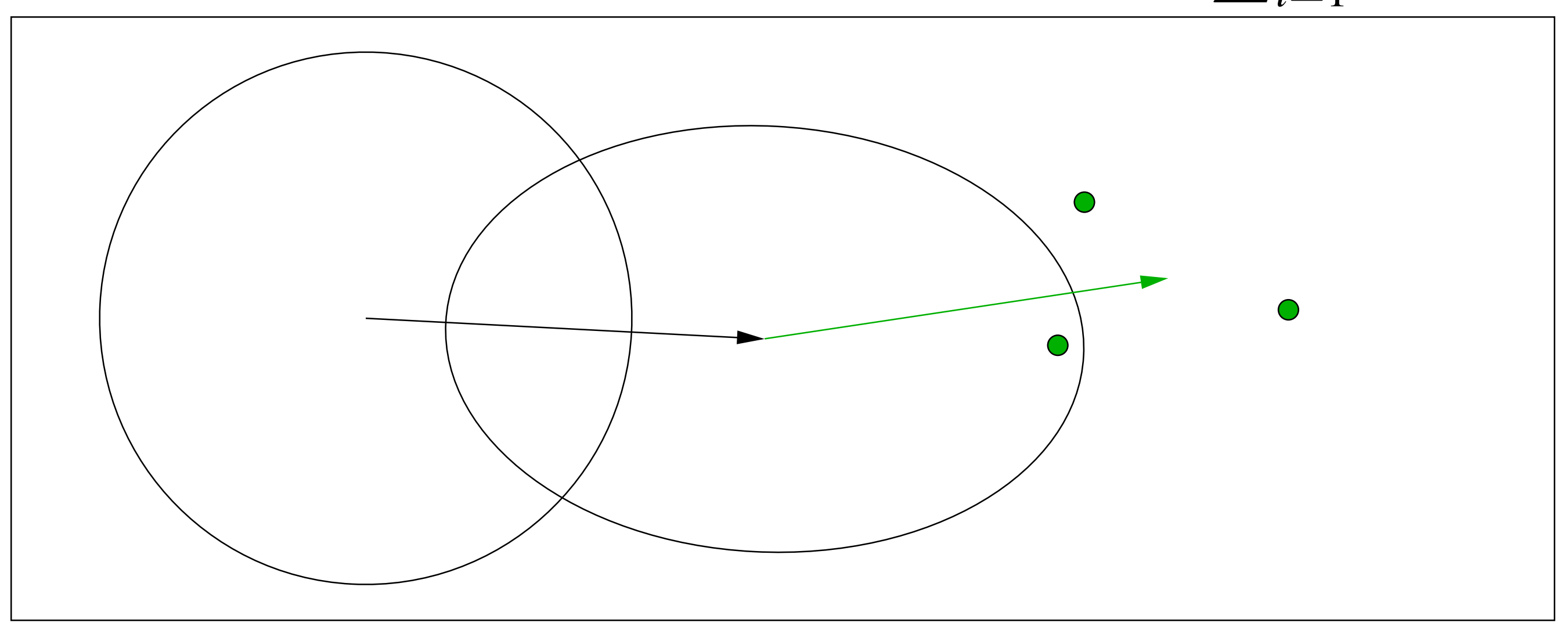

movement of the population mean $m$ 


\section{Covariance Matrix Adaptation}

\section{Rank-One Update}

$$
m \leftarrow m+\sigma \boldsymbol{y}_{w}, \quad \boldsymbol{y}_{w}=\sum_{i=1}^{\mu} w_{i} \boldsymbol{y}_{i: \lambda}, \quad \boldsymbol{y}_{i} \sim \mathcal{N}_{i}(\mathbf{0}, \mathbf{C})
$$

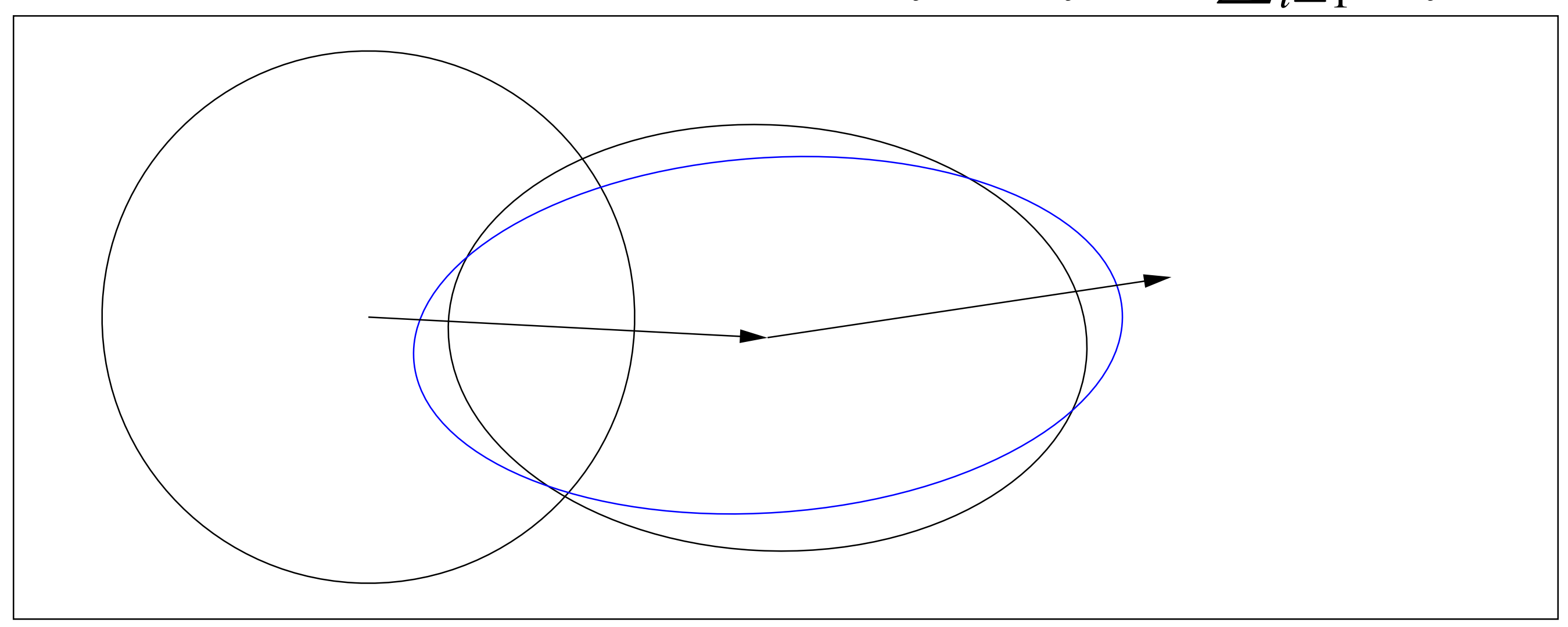

mixture of distribution C and step $\boldsymbol{y}_{w}$,

$\mathrm{C} \leftarrow 0.8 \times \mathbf{C}+0.2 \times \boldsymbol{y}_{w} \boldsymbol{y}_{w}^{\mathrm{T}}$ 


\section{Covariance Matrix Adaptation}

Rank-One Update

$$
m \leftarrow m+\sigma \boldsymbol{y}_{w}, \quad \boldsymbol{y}_{w}=\sum_{i=1}^{\mu} w_{i} \boldsymbol{y}_{i: \lambda}, \quad \boldsymbol{y}_{i} \sim \mathcal{N}_{i}(\mathbf{0}, \mathbf{C})
$$

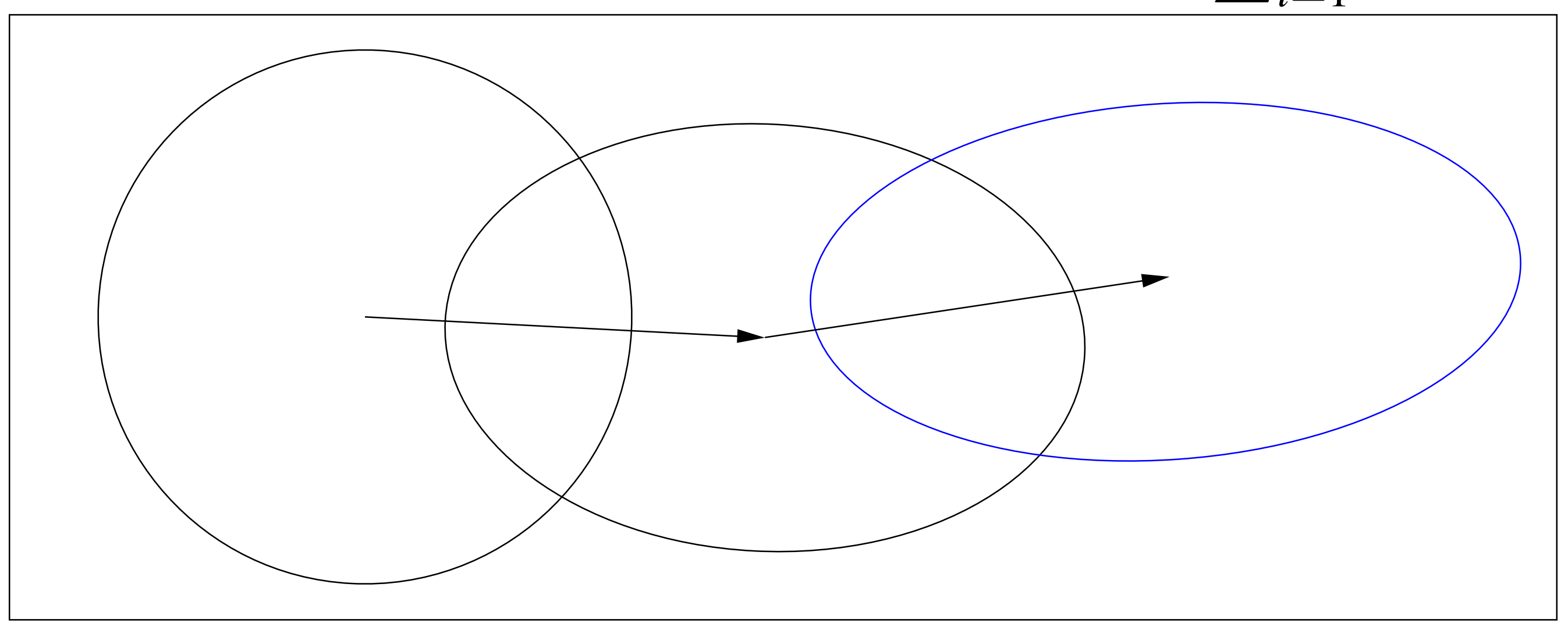

new distribution,

$\mathrm{C} \leftarrow 0.8 \times \mathbf{C}+0.2 \times \boldsymbol{y}_{w} \boldsymbol{y}_{w}^{\mathrm{T}}$

the ruling principle: the adaptation increases the likelihood of

successful steps, $\boldsymbol{y}_{w}$, to appear again

another viewpoint: the adaptation follows a natural gradient approximation of the expected fitness 


\section{Covariance Matrix Adaptation}

Rank-One Update

Initialize $m \in \mathbb{R}^{n}$, and $\mathbf{C}=\mathbf{I}$, set $\sigma=1$, learning rate $c_{\mathrm{cov}} \approx 2 / n^{2}$ While not terminate

$$
\begin{aligned}
& \boldsymbol{x}_{i}=m+\sigma \boldsymbol{y}_{i}, \quad \boldsymbol{y}_{i} \sim \mathcal{N}_{i}(\mathbf{0}, \mathbf{C}) \\
& m \leftarrow m+\sigma \boldsymbol{y}_{w} \quad \text { where } \boldsymbol{y}_{w}=\sum_{i=1}^{\mu} w_{i} \boldsymbol{y}_{i: \lambda} \\
& \mathrm{C} \leftarrow\left(1-c_{\mathrm{cov}}\right) \mathrm{C}+c_{\mathrm{cov}} \mu_{w} \underbrace{\boldsymbol{y}_{w} \boldsymbol{y}_{w}^{\mathrm{T}}}_{\text {rank-one }} \quad \text { where } \mu_{w}=\frac{1}{\sum_{i=1}^{\mu} w_{i}{ }^{2}} \geq 1
\end{aligned}
$$

The rank-one update has been found independently in several domains ${ }^{6} 789$

${ }^{6}$ Kjellström\&Taxén 1981. Stochastic Optimization in System Design, IEEE TCS

7 Hansen\&Ostermeier 1996. Adapting arbitrary normal mutation distributions in evolution strategies: The covariance matrix adaptation, ICEC

${ }^{8}$ Ljung 1999. System Identification: Theory for the User

9 Haario et al 2001. An adaptive Metropolis algorithm, JSTOR 
covariance matrix adaptation

$$
\mathrm{C} \leftarrow\left(1-c_{\mathrm{cov}}\right) \mathrm{C}+c_{\mathrm{cov}} \mu_{w} \boldsymbol{y}_{w} \boldsymbol{y}_{w}^{\mathrm{T}}
$$

- learns all pairwise dependencies between variables

off-diagonal entries in the covariance matrix reflect the dependencies

- conducts a principle component analysis (PCA) of steps $\boldsymbol{y}_{w}$, sequentially in time and space

eigenvectors of the covariance matrix $\mathrm{C}$ are the principle components / the principle axes of the mutation ellipsoid

- learns a new rotated problem representation components are independent (only) in the new representation

- learns a new (Mahalanobis) metric

variable metric method

- approximates the inverse Hessian on quadratic functions transformation into the sphere function - for $\mu=1$ : conducts a natural gradient ascent on the distribution $\mathcal{N}$ entirely independent of the given coordinate system 


\section{Invariance Under Rigid Search Space Transformation}

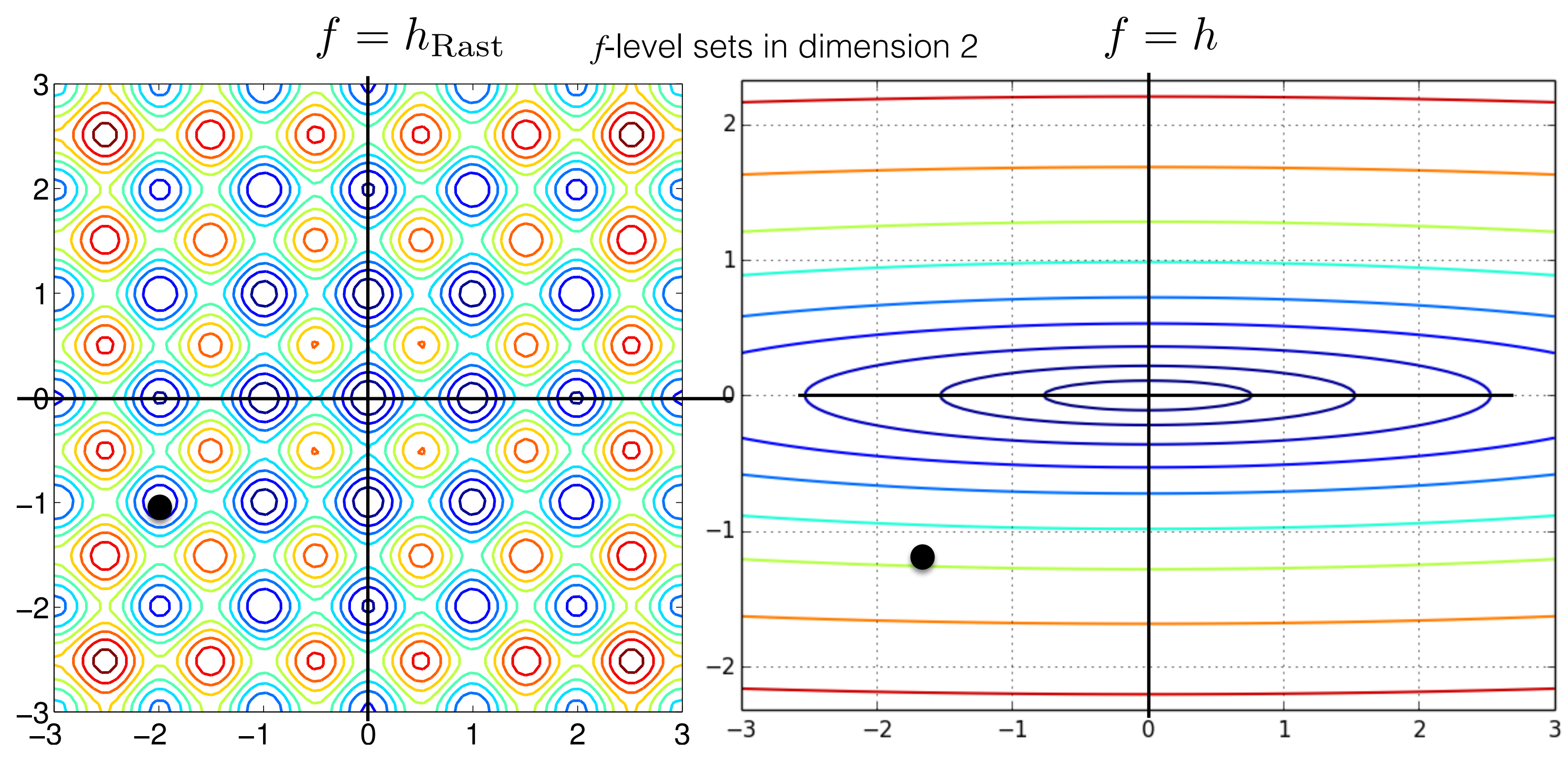

for example, invariance under search space rotation (separable $\Leftrightarrow$ non-separable) 


\section{Invariance Under Rigid Search Space Transformation}

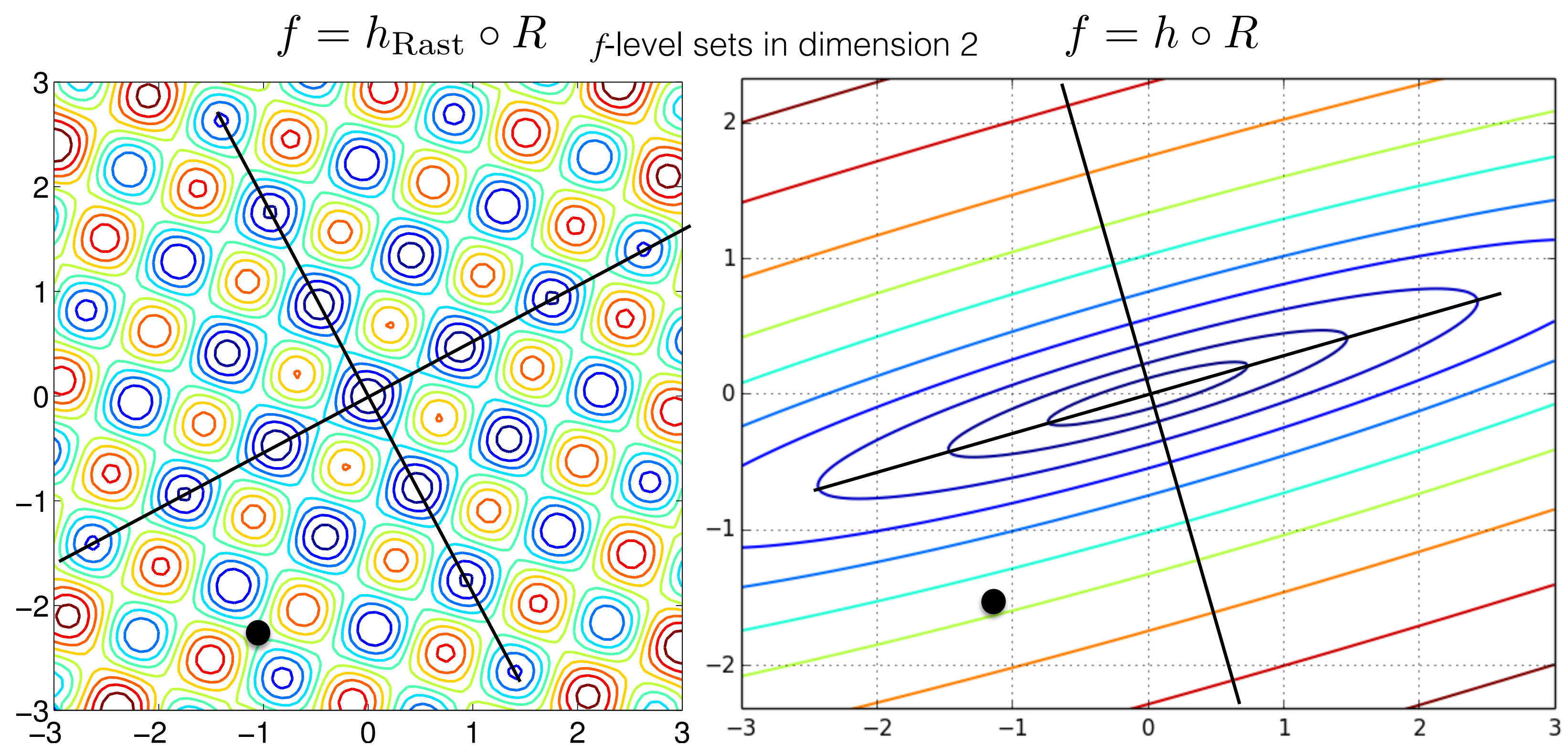

for example, invariance under search space rotation (separable $\Leftrightarrow$ non-separable) 


\section{Cumulation}

The Evolution Path

\section{Evolution Path}

Conceptually, the evolution path is the search path the strategy takes over a number of generation steps. It can be expressed as a sum of consecutive steps of the mean $m$.

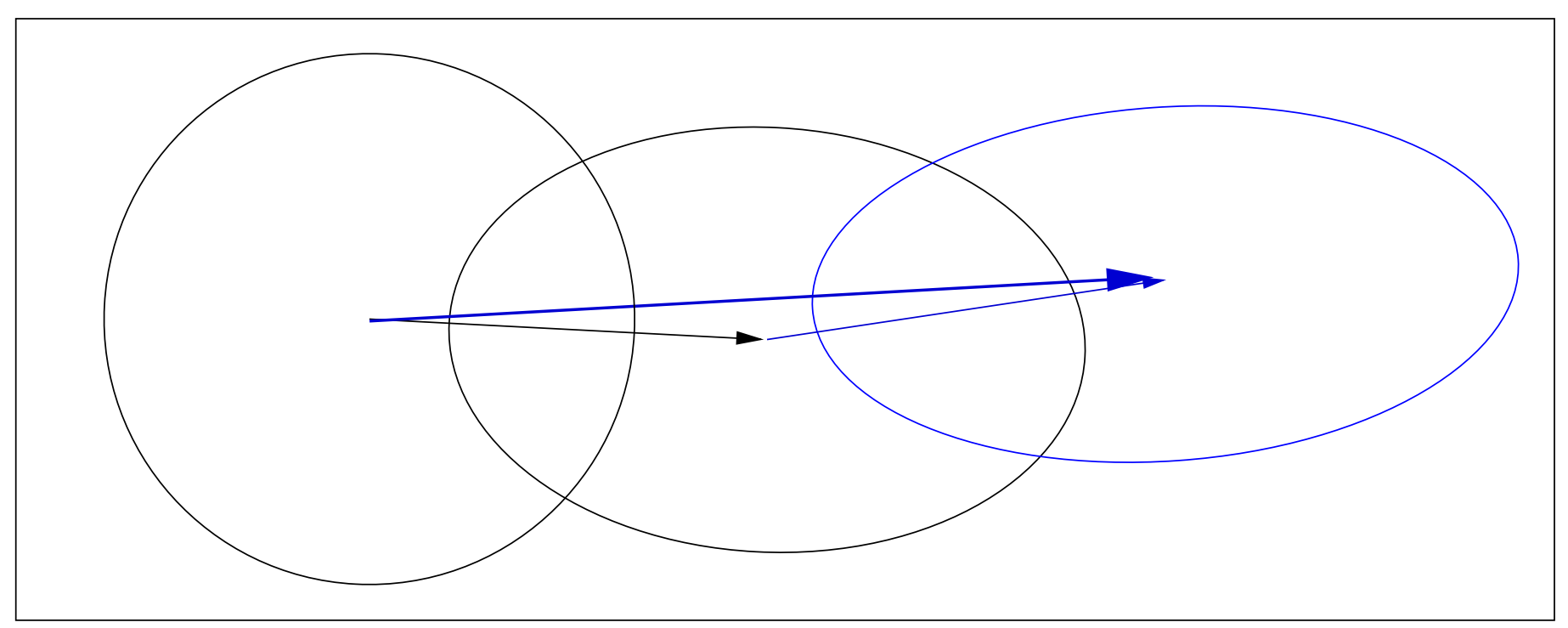

An exponentially weighted sum of steps $\boldsymbol{y}_{w}$ is used

$$
p_{\mathrm{c}} \propto \sum_{i=0}^{g} \underbrace{\left(1-c_{\mathbf{c}}\right)^{g-i}}_{\substack{\text { exponentially } \\ \text { fading weights }}} \boldsymbol{y}_{w}^{(i)}
$$

The recursive construction of the evolution path (cumulation):
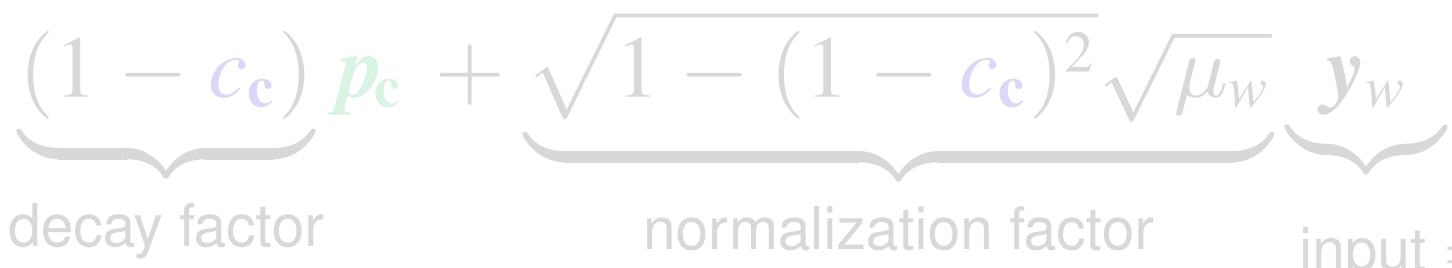


\section{Cumulation}

The Evolution Path

\section{Evolution Path}

Conceptually, the evolution path is the search path the strategy takes over a number of generation steps. It can be expressed as a sum of consecutive steps of the mean $m$.

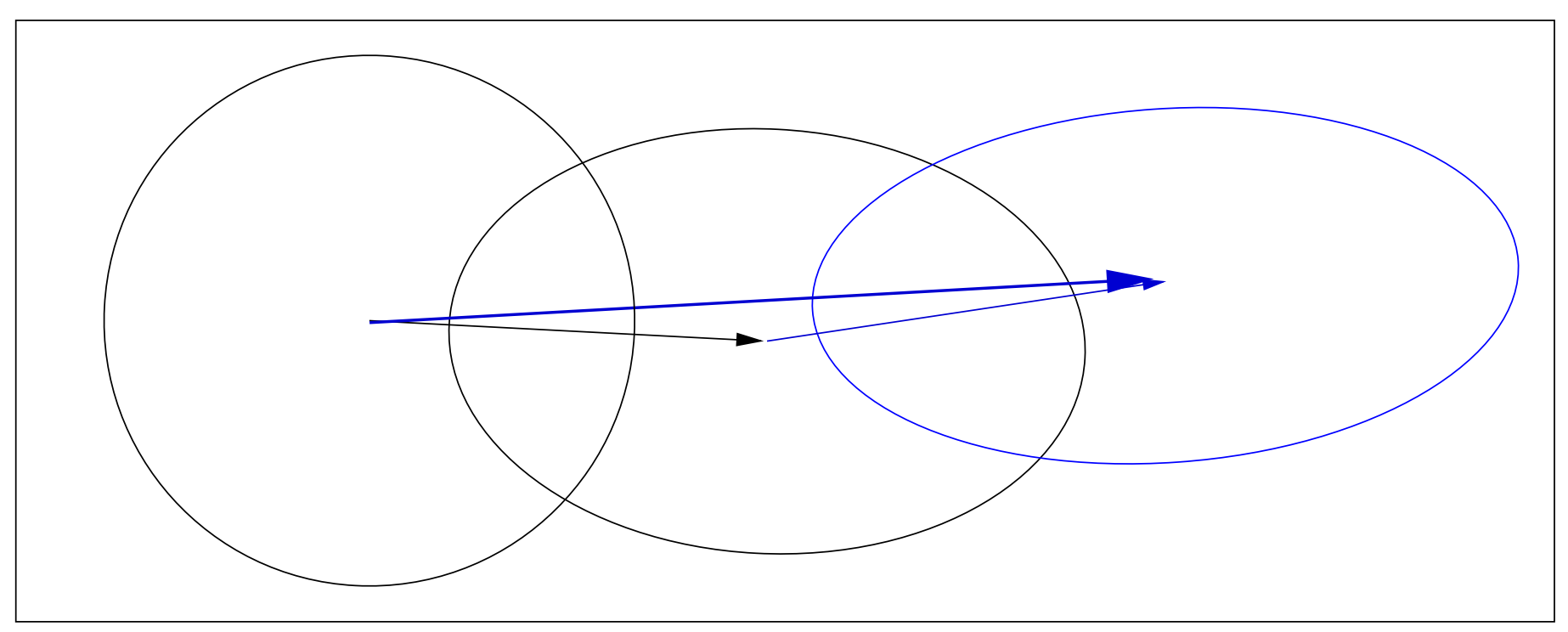

An exponentially weighted sum of steps $\boldsymbol{y}_{w}$ is used

$$
p_{\mathrm{c}} \propto \sum_{i=0}^{g} \underbrace{\left(1-c_{\mathbf{c}}\right)^{g-i}}_{\substack{\text { exponentially } \\ \text { fading weights }}} \boldsymbol{y}_{w}^{(i)}
$$

The recursive construction of the evolution path (cumulation):

$$
p_{\mathrm{c}} \leftarrow \underbrace{\left(1-c_{\mathbf{c}}\right)}_{\text {decay factor }} p_{\mathrm{c}}+\underbrace{\sqrt{1-\left(1-c_{\mathbf{c}}\right)^{2}} \sqrt{\mu_{w}}}_{\text {normalization factor }} \underbrace{\boldsymbol{y}_{w}}_{\text {input }=\frac{m-m_{\text {old }}}{\sigma}}
$$

where $\mu_{w}=\frac{1}{\sum w_{i}^{2}}, c_{\mathbf{c}} \ll 1$. History information is accumulated in the evolution path. 
"Cumulation" is a widely used technique and also know as

- exponential smoothing in time series, forecasting

- exponentially weighted mooving average

- iterate averaging in stochastic approximation

- momentum in the back-propagation algorithm for ANNs

- ...

"Cumulation" conducts a low-pass filtering, but there is more to it... 


\section{Cumulation \\ $\mathbf{C} \leftarrow\left(1-c_{\mathrm{cov}}\right) \mathbf{C}+c_{\mathrm{cov}} \mu_{w} \boldsymbol{y}_{w} \boldsymbol{y}_{w}^{\mathrm{T}}$}

Utilizing the Evolution Path

We used $\boldsymbol{y}_{w} \boldsymbol{y}_{w}^{T}$ for updating C. Because $\boldsymbol{y}_{w} \boldsymbol{y}_{w}^{\mathrm{T}}=-\boldsymbol{y}_{w}\left(-\boldsymbol{y}_{w}\right)^{\mathrm{T}}$ the sign of $\boldsymbol{y}_{w}$ is lost.

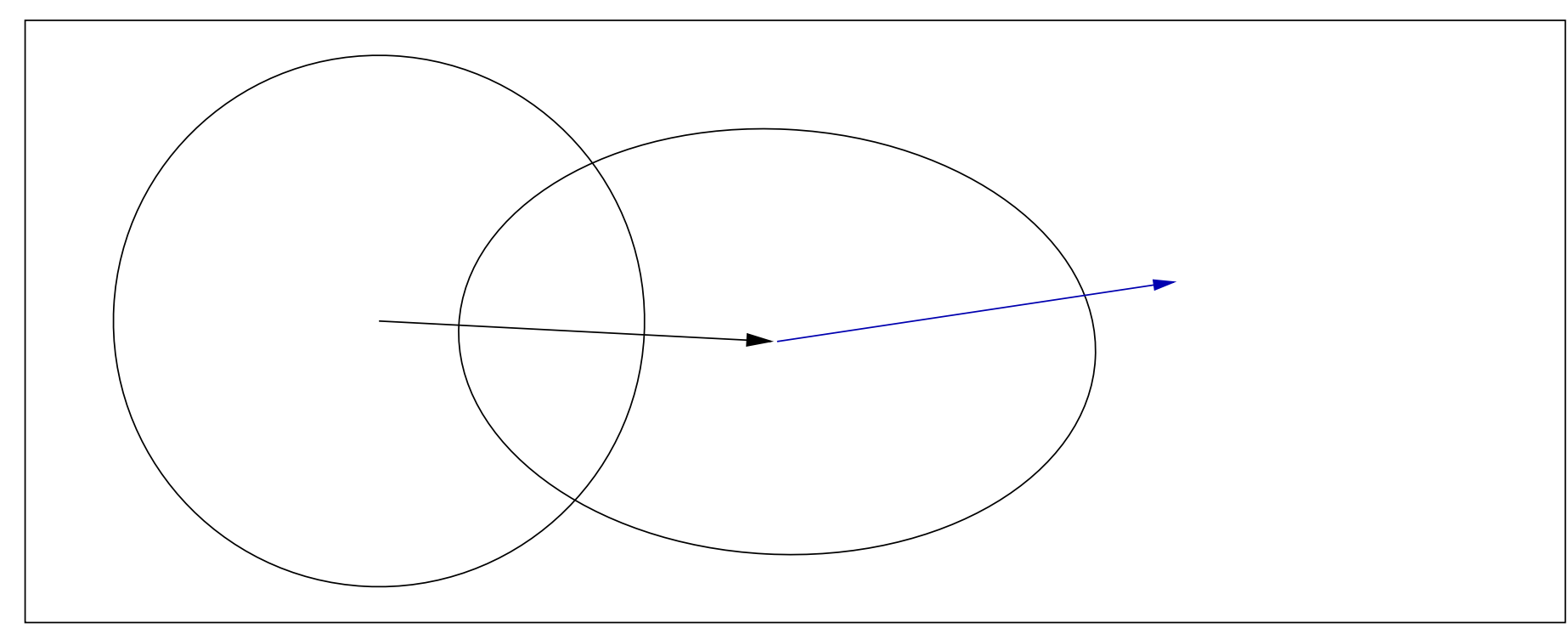

The sign information (signifying correlation between steps) is (re-)introduced by using the evolution path.

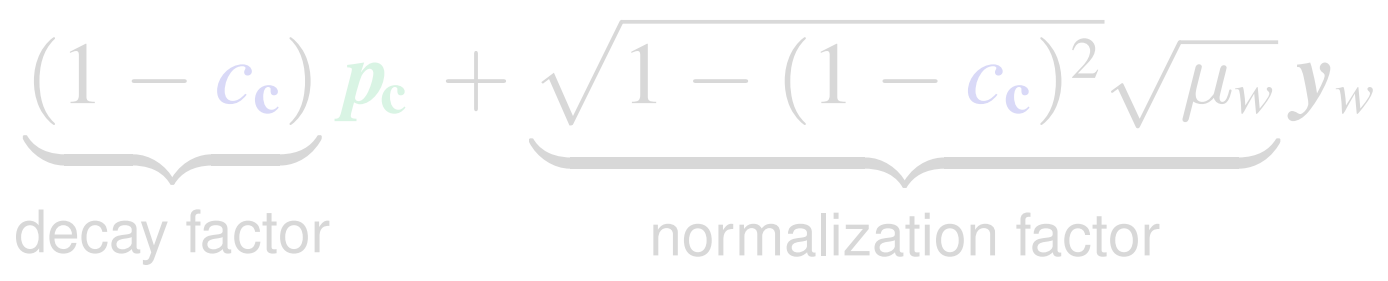




\section{Cumulation \\ $\mathbf{C} \leftarrow\left(1-c_{\mathrm{cov}}\right) \mathbf{C}+c_{\mathrm{cov}} \mu_{w} \boldsymbol{y}_{w} \boldsymbol{y}_{w}^{\mathrm{T}}$}

Utilizing the Evolution Path

We used $\boldsymbol{y}_{w} \boldsymbol{y}_{w}^{T}$ for updating C. Because $\boldsymbol{y}_{w} \boldsymbol{y}_{w}^{\mathrm{T}}=-\boldsymbol{y}_{w}\left(-\boldsymbol{y}_{w}\right)^{\mathrm{T}}$ the sign of $\boldsymbol{y}_{w}$ is lost.

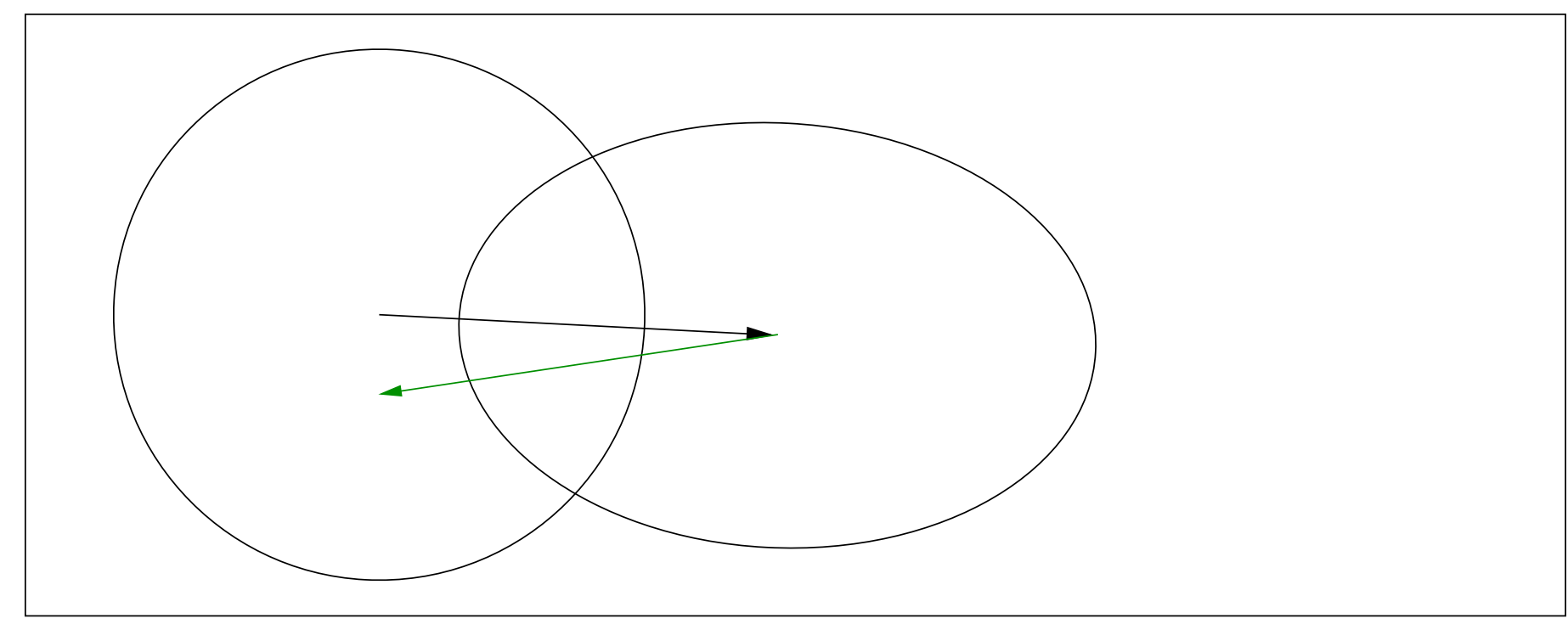

The sign information (signifying correlation between steps) is (re-)introduced by using the evolution path.

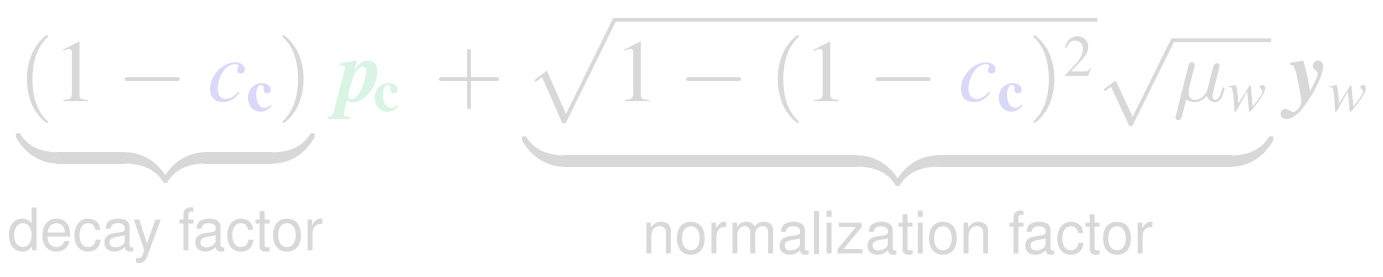




\section{Cumulation \\ $\mathrm{C} \leftarrow\left(1-c_{\mathrm{cov}}\right) \mathrm{C}+c_{\mathrm{cov}} \mu_{w} \boldsymbol{y}_{w} \boldsymbol{y}_{w}^{\mathrm{T}}$}

Utilizing the Evolution Path

We used $\boldsymbol{y}_{w} \boldsymbol{y}_{w}^{\mathrm{T}}$ for updating C. Because $\boldsymbol{y}_{w} \boldsymbol{y}_{w}^{\mathrm{T}}=-\boldsymbol{y}_{w}\left(-\boldsymbol{y}_{w}\right)^{\mathrm{T}}$ the sign of $\boldsymbol{y}_{w}$ is lost.

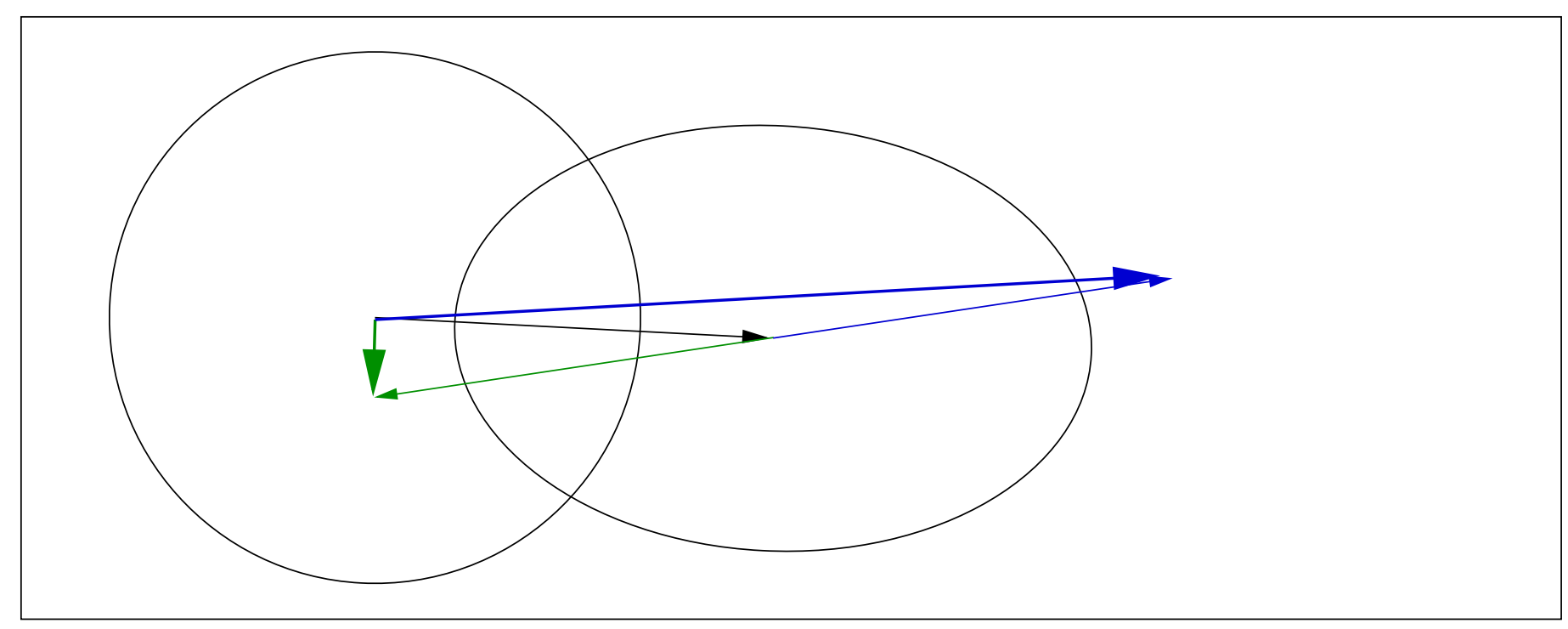

The sign information (signifying correlation between steps) is (re-)introduced by using the evolution path.

$$
\begin{aligned}
& p_{\mathrm{c}} \leftarrow \underbrace{\left(1-c_{\mathbf{c}}\right)}_{\text {decay factor }} p_{\mathrm{c}}+\underbrace{\sqrt{1-\left(1-c_{\mathbf{c}}\right)^{2}} \sqrt{\mu_{w}}}_{\text {normalization factor }} \boldsymbol{y}_{w} \\
& \mathrm{C} \leftarrow\left(1-c_{\text {cov }}\right) \mathrm{C}+c_{\text {cov }} \underbrace{p_{\mathrm{c}} p_{\mathrm{c}}^{\mathrm{T}}}_{\text {rank-one }}
\end{aligned}
$$

where $\mu_{w}=\frac{1}{\sum w_{i}^{2}}, c_{\mathrm{cov}} \ll c_{\mathbf{c}} \ll 1$ such that $1 / c_{\mathbf{c}}$ is the "backward time horizon". 
Using an evolution path for the rank-one update of the covariance matrix reduces the number of function evaluations to adapt to a straight ridge from about $\mathcal{O}\left(n^{2}\right)$ to $\mathcal{O}(n) .{ }^{(a)}$

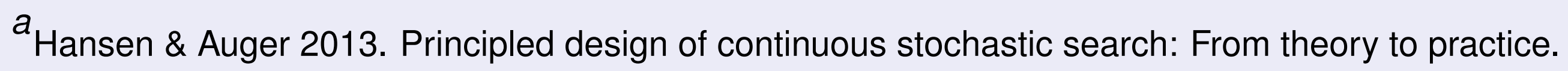

Number of $f$-evaluations divided by dimension on the cigar function $f(\boldsymbol{x})=x_{1}^{2}+10^{6} \sum_{i=2}^{n} x_{i}^{2}$

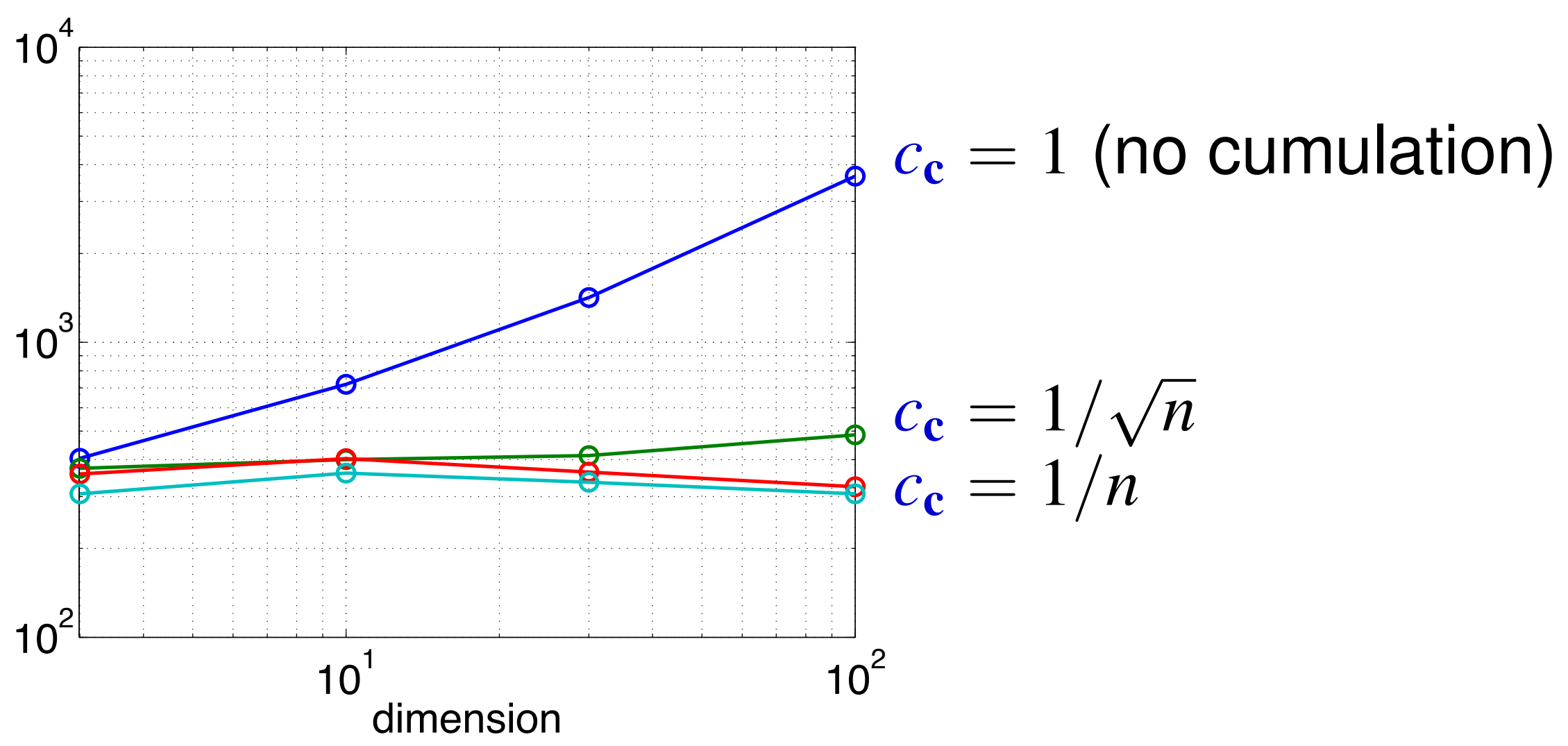

The overall model complexity is $n^{2}$ but important parts of the model can be learned in time of order $n$ 


\section{Rank- $\mu$ Update}

$$
\begin{aligned}
& \boldsymbol{x}_{i}=m+\sigma \boldsymbol{y}_{i}, \quad \boldsymbol{y}_{i} \sim \mathcal{N}_{i}(\mathbf{0}, \mathbf{C}), \\
& m \leftarrow m+\sigma \boldsymbol{y}_{w}
\end{aligned}
$$

The rank- $\mu$ update extends the update rule for large population sizes $\lambda$ using $\mu>1$ vectors to update $\mathrm{C}$ at each generation step. 

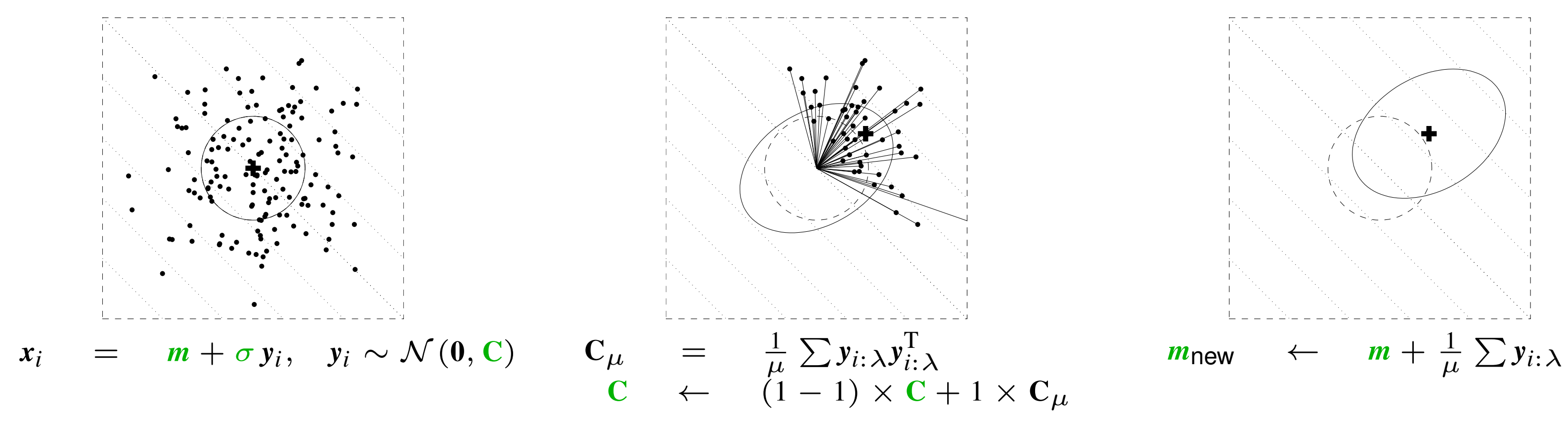

new distribution

sampling of $\lambda=150$ calculating $\mathrm{C}$ where solutions where $\mathbf{C}=\mathbf{I}$ and $\sigma=1$

$$
\begin{gathered}
\mu=50, \\
w_{1}=\cdots=w_{\mu}=\frac{1}{\mu}, \\
\text { and } c_{\mathrm{cov}}=1
\end{gathered}
$$




\section{Rank- $\mu$ CMA versus Estimation of Multivariate Normal Algorithm EMNA global $^{11}$}

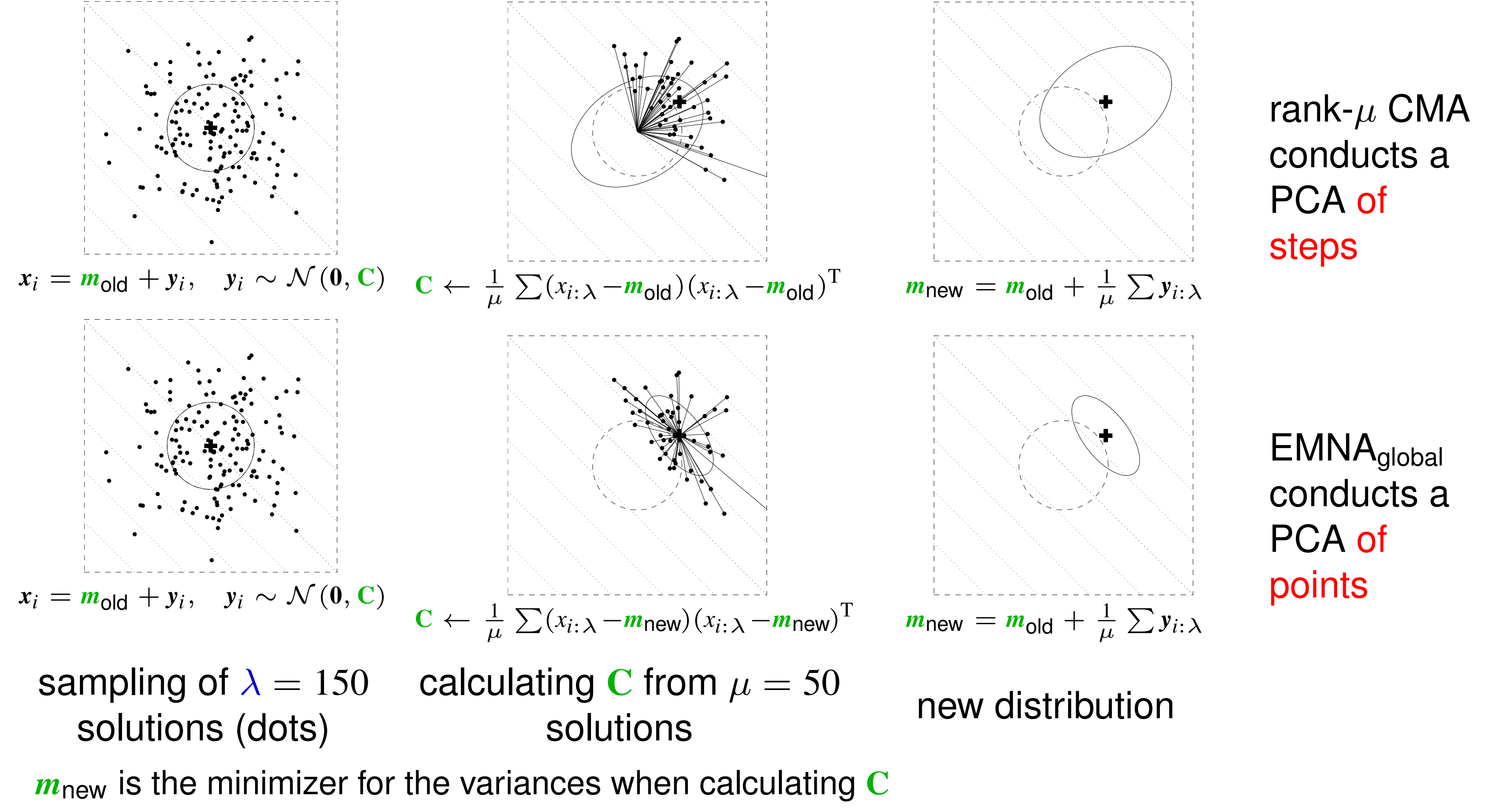

11 Hansen, N. (2006). The CMA Evolution Strategy: A Comparing Review. In J.A. Lozano, P. Larranga, I. Inza and E. Bengoetxea (Eds.). Towards a new evolutionary computation. Advances in estimation of distribution alqorithms. pp. 75-102 
The rank- $\mu$ update

- increases the possible learning rate in large populations

roughly from $2 / n^{2}$ to $\mu_{w} / n^{2}$

- can reduce the number of necessary generations roughly from $\mathcal{O}\left(n^{2}\right)$ to $\mathcal{O}(n)^{(12)}$

given $\mu_{w} \propto \lambda \propto n$

Therefore the rank- $\mu$ update is the primary mechanism whenever a large population size is used

$$
\text { say } \lambda \geq 3 n+10
$$

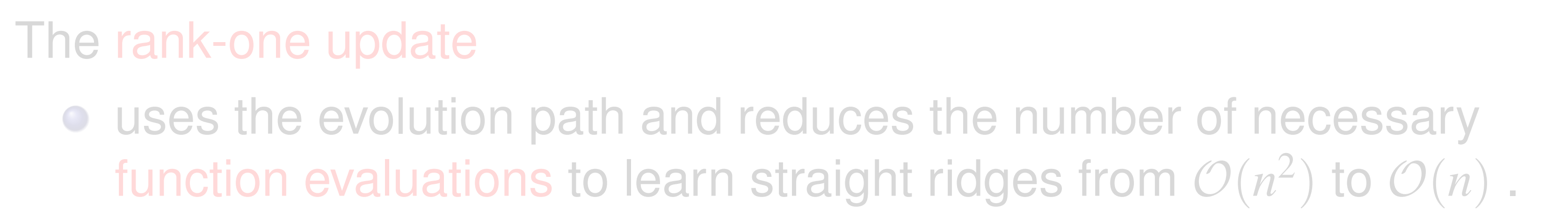

Rank-one update and rank- $\mu$ update can be combined

${ }^{12}$ Hansen, Müller, and Koumoutsakos 2003. Reducing the Time Complexity of the Derandomized Evolution Strategy with Covariance Matrix Adaptation (CMA-ES). Evolutionary Computation, 11(1), pp. 1-18 
The rank- $\mu$ update

- increases the possible learning rate in large populations

roughly from $2 / n^{2}$ to $\mu_{w} / n^{2}$

- can reduce the number of necessary generations roughly from $\mathcal{O}\left(n^{2}\right)$ to $\mathcal{O}(n)^{(12)}$

given $\mu_{w} \propto \lambda \propto n$

Therefore the rank- $\mu$ update is the primary mechanism whenever a large population size is used

$$
\text { say } \lambda \geq 3 n+10
$$

The rank-one update

- uses the evolution path and reduces the number of necessary function evaluations to learn straight ridges from $\mathcal{O}\left(n^{2}\right)$ to $\mathcal{O}(n)$.

${ }^{12}$ Hansen, Müller, and Koumoutsakos 2003. Reducing the Time Complexity of the Derandomized Evolution Strategy with Covariance Matrix Adaptation (CMA-ES). Evolutionary Computation, 11(1), pp. 1-18 
The rank- $\mu$ update

- increases the possible learning rate in large populations

roughly from $2 / n^{2}$ to $\mu_{w} / n^{2}$

- can reduce the number of necessary generations roughly from $\mathcal{O}\left(n^{2}\right)$ to $\mathcal{O}(n)$

given $\mu_{w} \propto \lambda \propto n$

Therefore the rank- $\mu$ update is the primary mechanism whenever a large population size is used

$$
\text { say } \lambda \geq 3 n+10
$$

The rank-one update

- uses the evolution path and reduces the number of necessary function evaluations to learn straight ridges from $\mathcal{O}\left(n^{2}\right)$ to $\mathcal{O}(n)$.

Rank-one update and rank- $\mu$ update can be combined

${ }^{12}$ Hansen, Müller, and Koumoutsakos 2003. Reducing the Time Complexity of the Derandomized Evolution Strategy with Covariance Matrix Adaptation (CMA-ES). Evolutionary Computation, 11(1), pp. 1-18 


\section{Rank-one update}

best $\mathrm{f}$-value
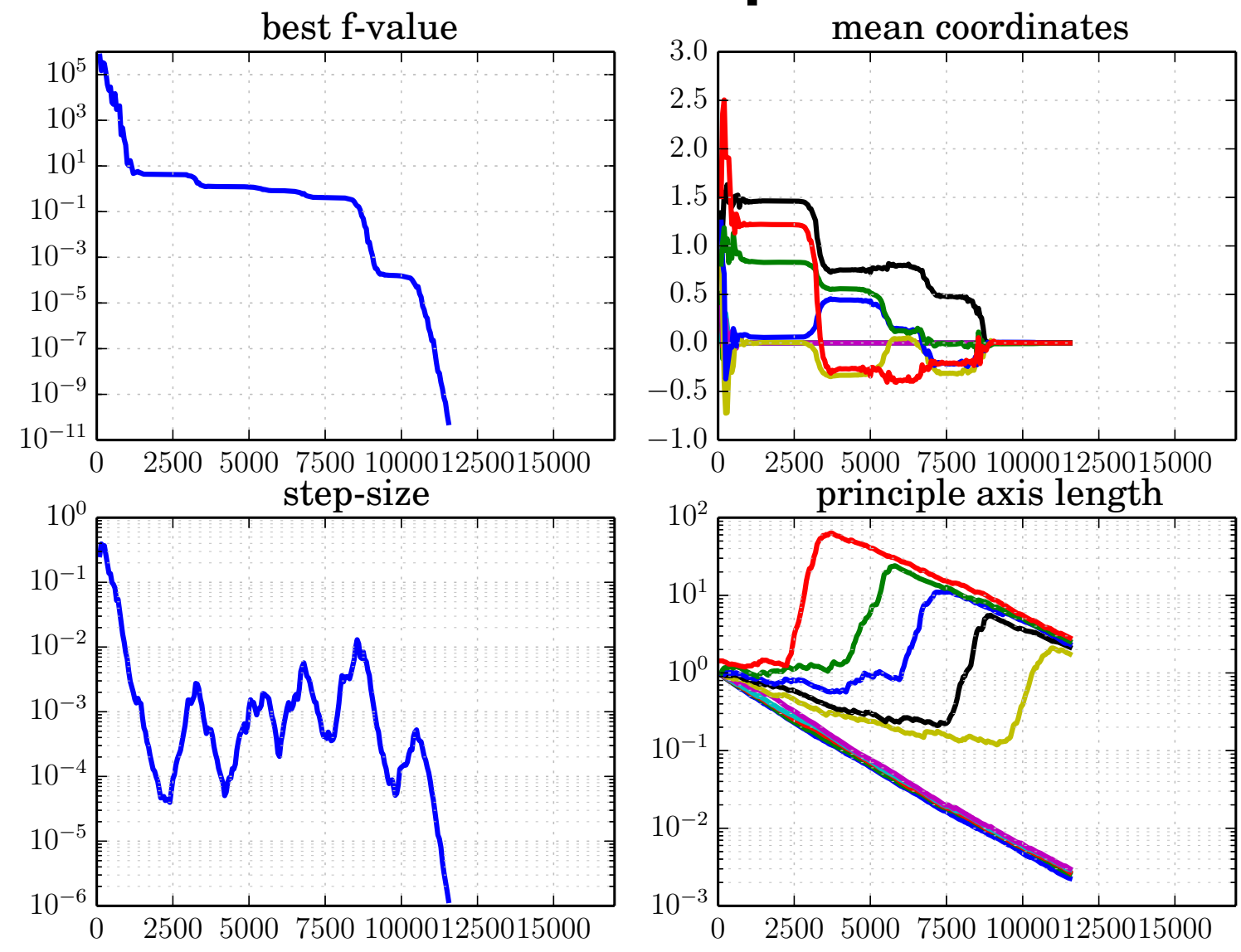

Rank- $\mu$ update

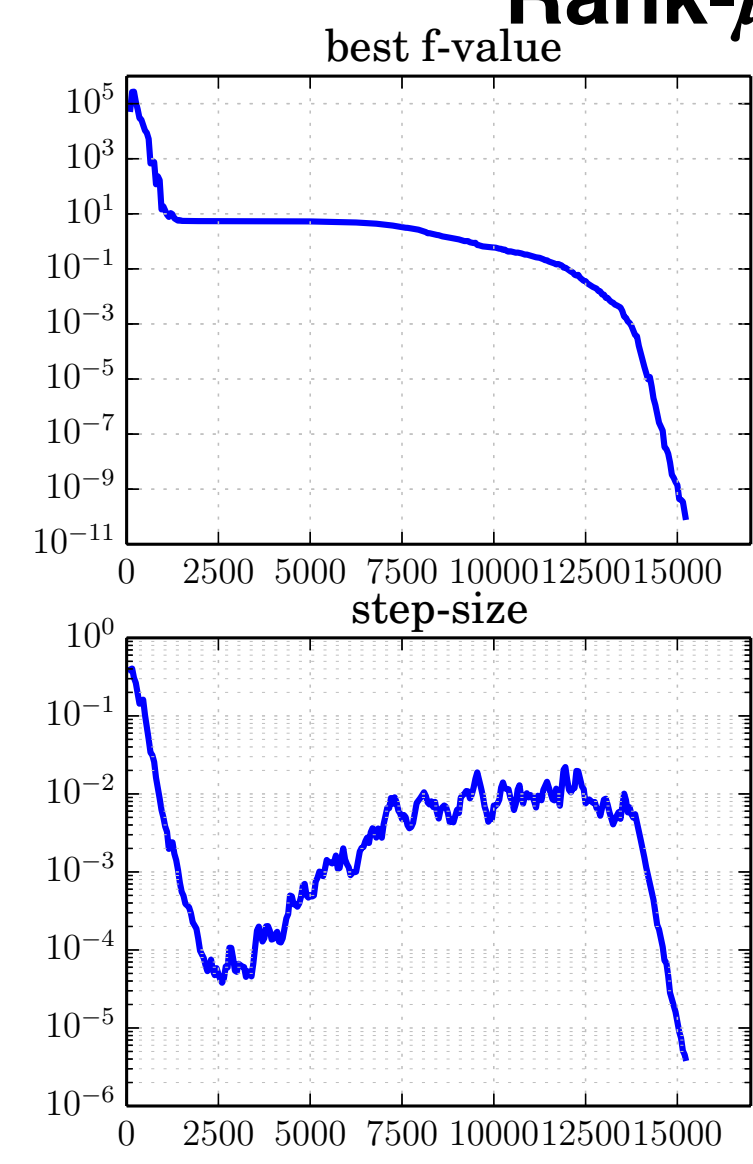

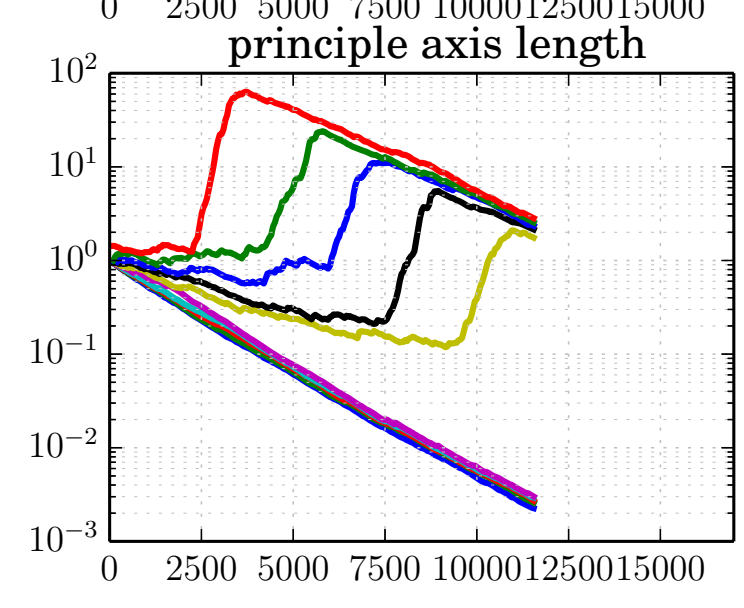
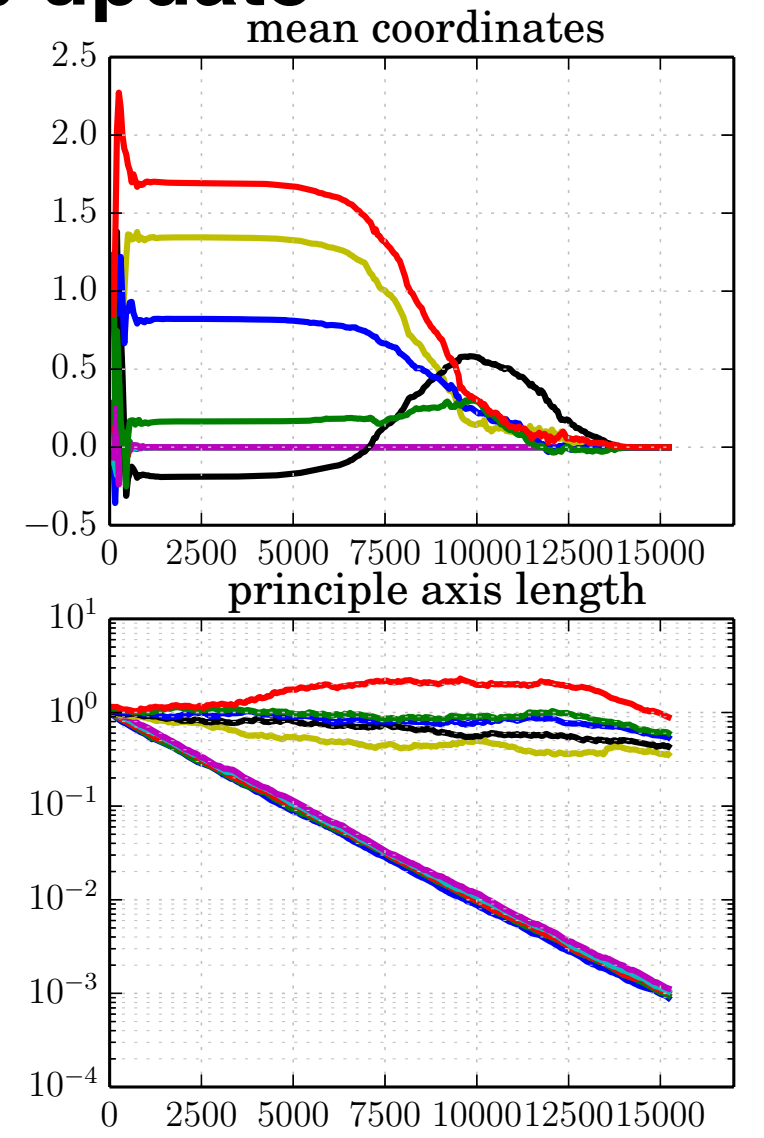

Hybridl (combined) update

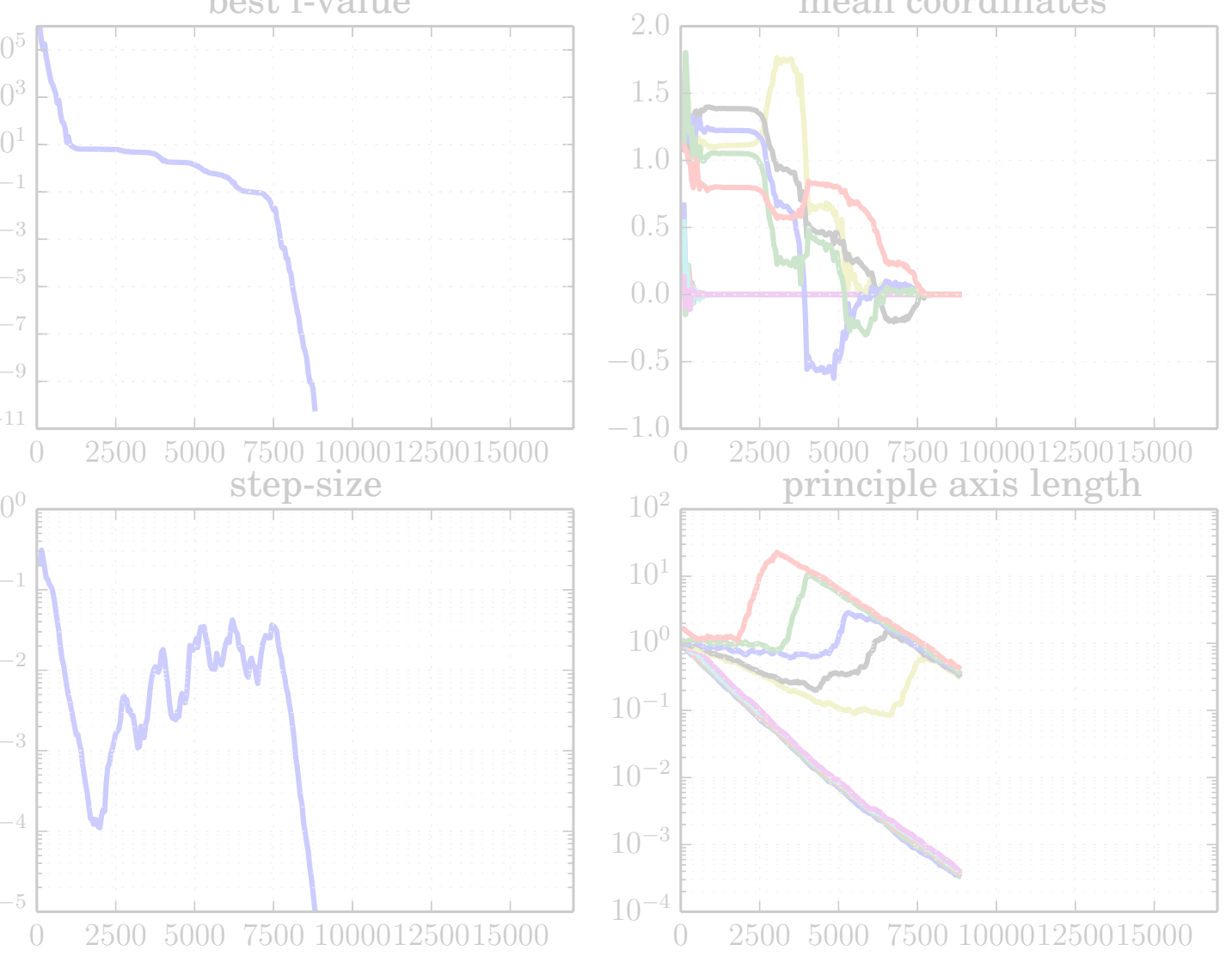

$f_{\text {TwoAxes }}(x)=\sum_{i=1}^{5} x_{i}^{2}+10^{6} \sum_{i=6}^{10} x_{i}^{2}$

$\lambda=10$ (default for $N=10$ ) 


\section{Rank-one update}

best f-value
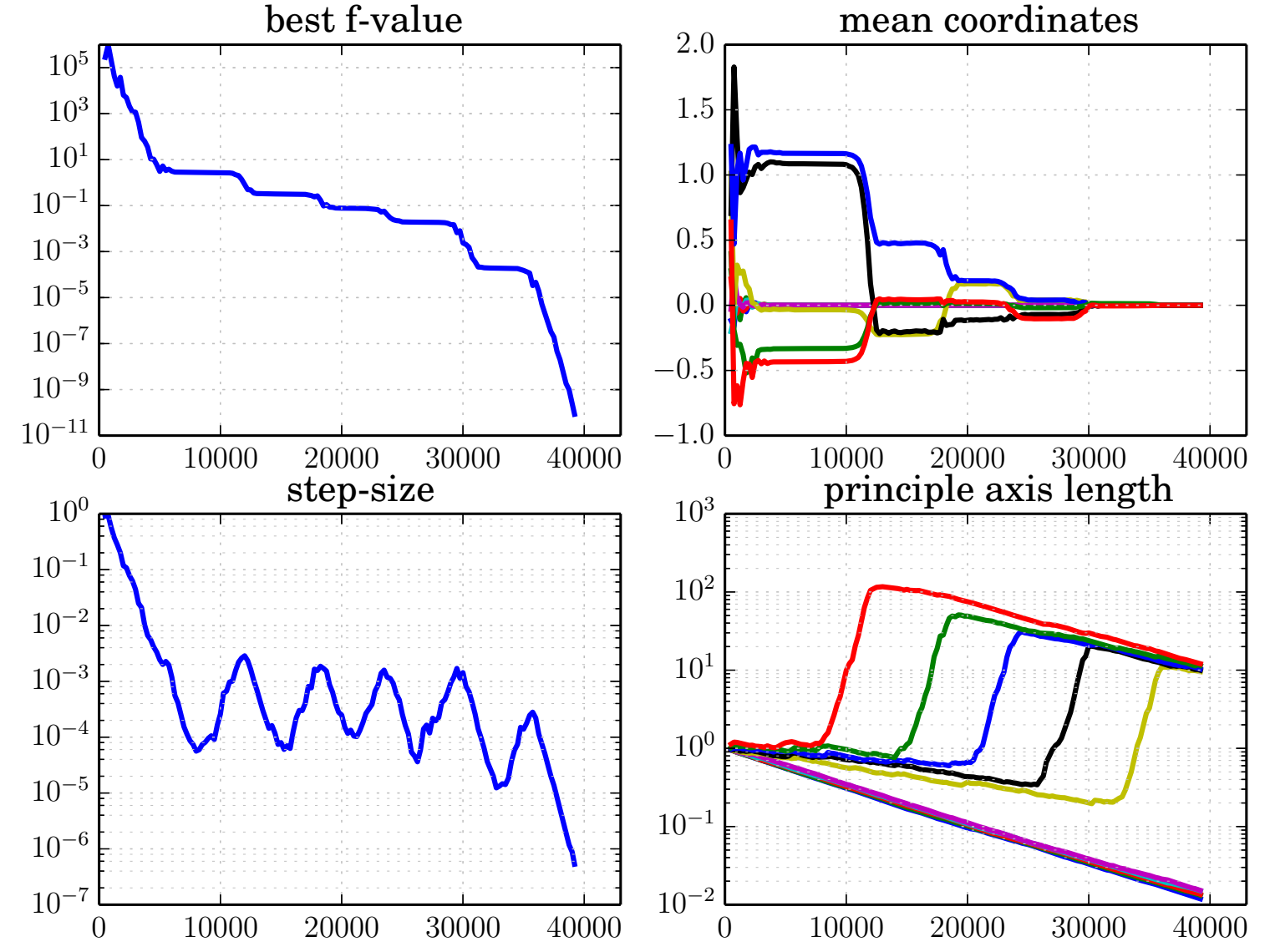

Rank- $\mu$ update
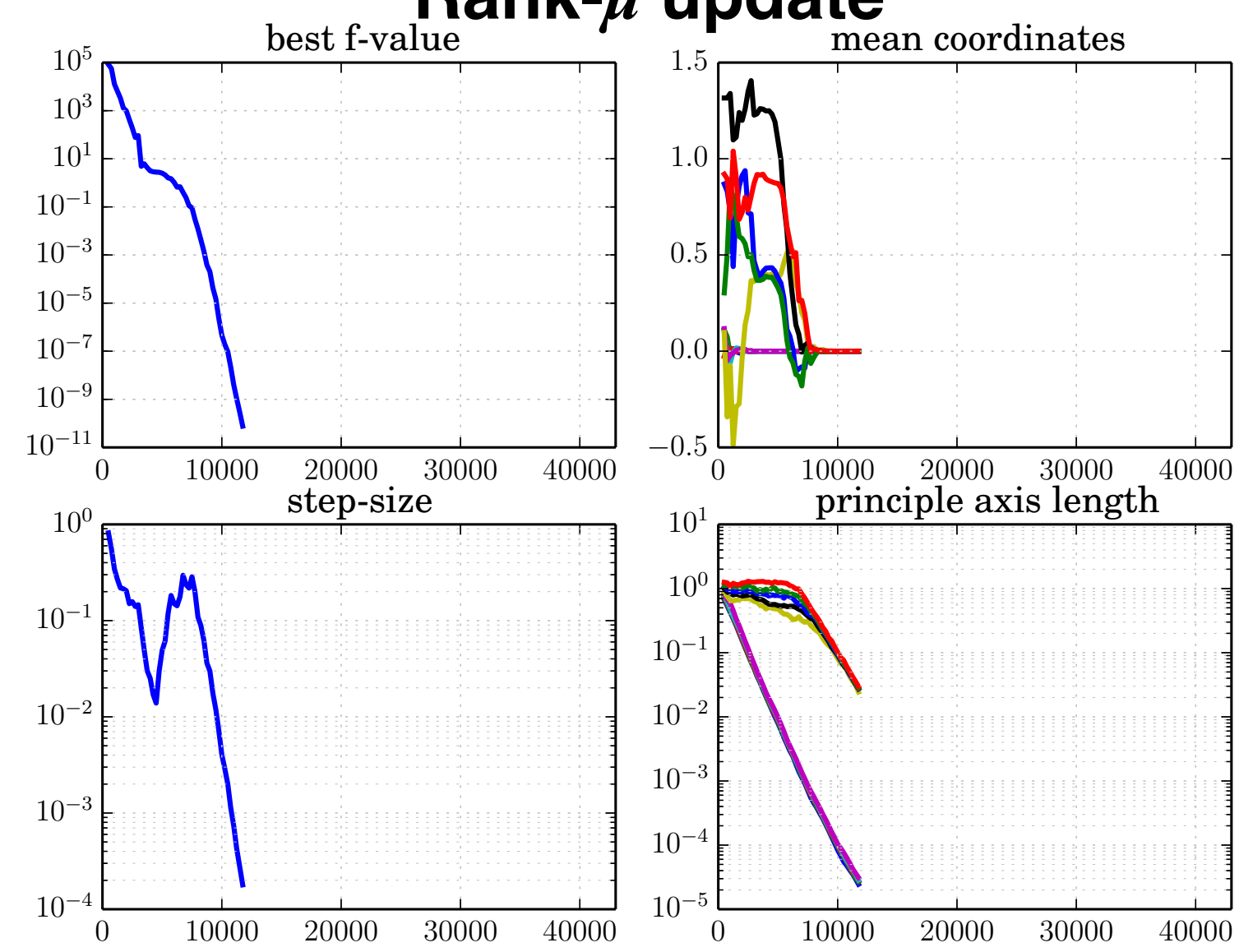

Hybridl (combined) update

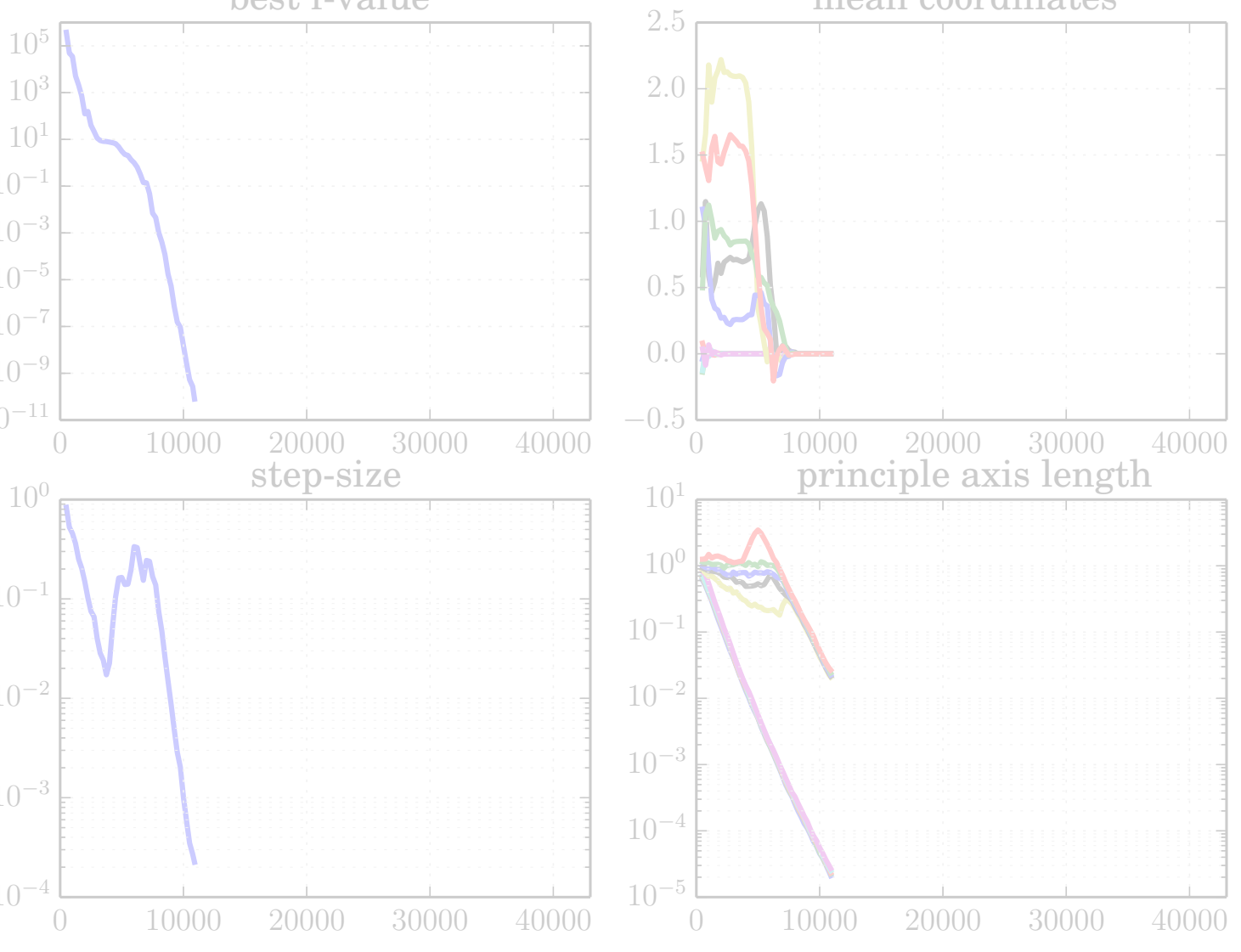

$f_{\text {TwoAxes }}(x)=\sum_{i=1}^{5} x_{i}^{2}+10^{6} \sum_{i=6}^{10} x_{i}^{2}$

$\lambda=50$ 


\section{Different Types of III-Conditioning $\quad$ (a: Axes Ratio = 10)}

Cigar Type:

1 long axis $=\mathrm{n}-1$ short axes

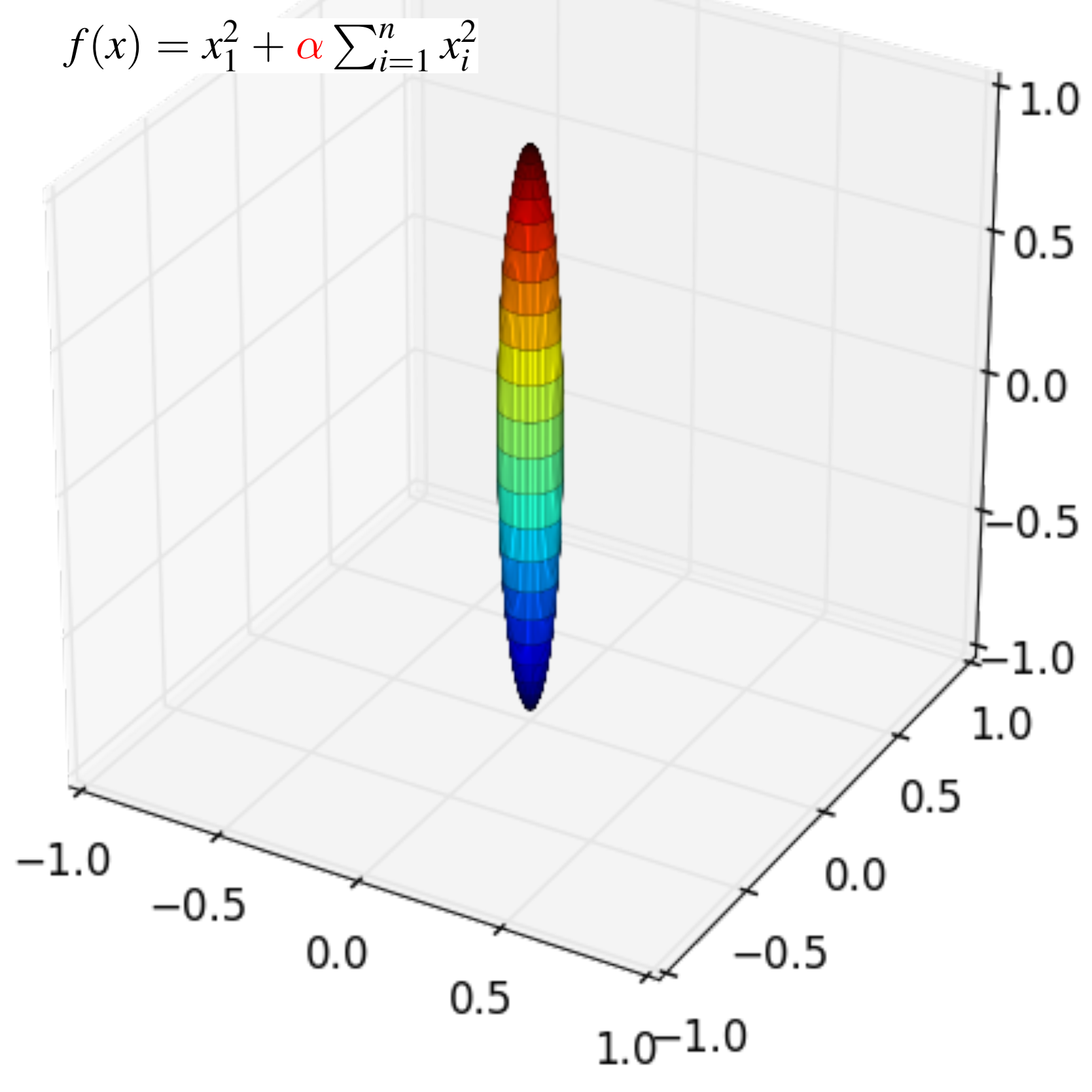

Discus Type:

1 short axis $=\mathrm{n}-1$ long axes

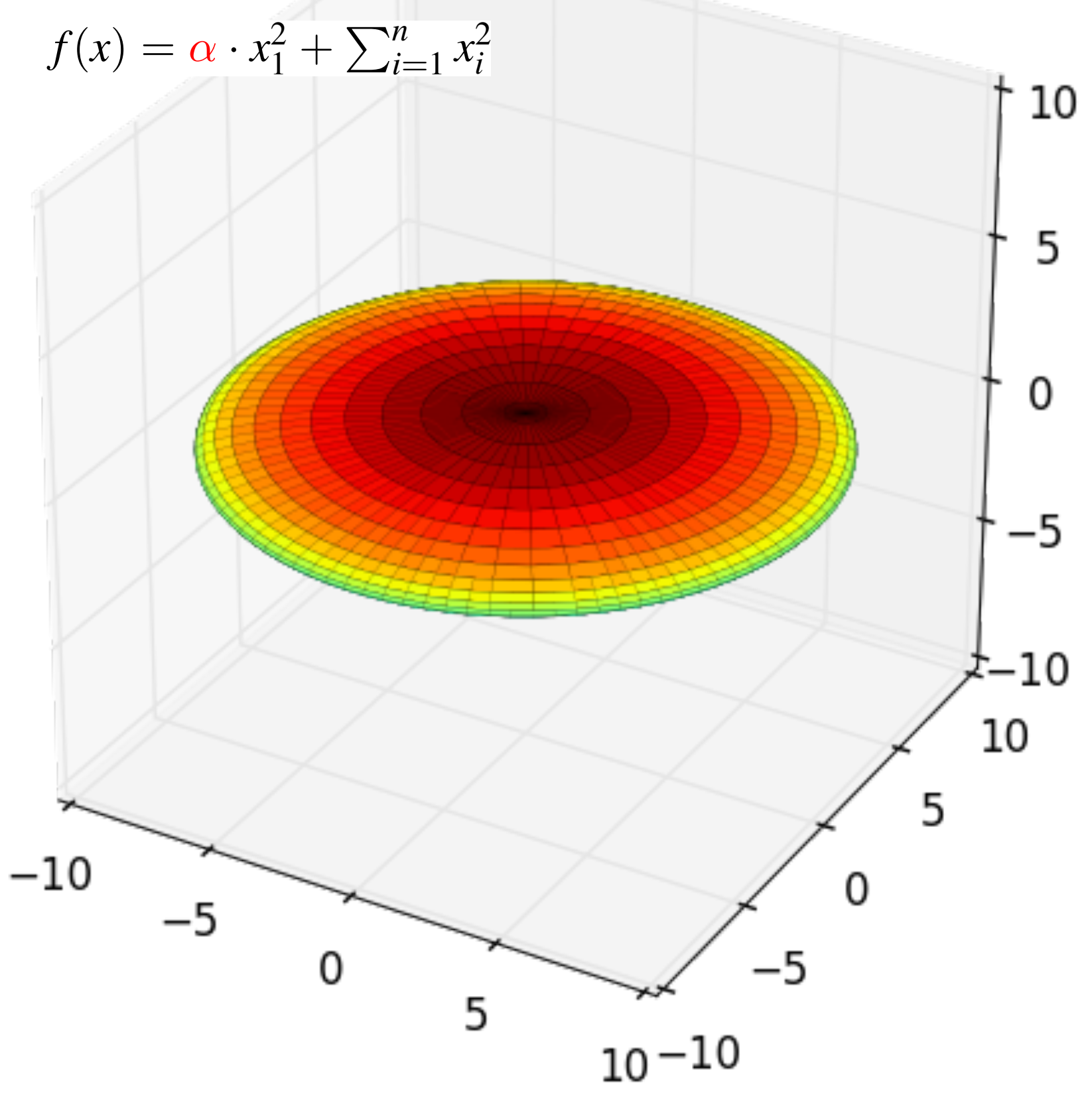




\section{Active Update}

utilize negative weights [Jastrebski and Arnold, 2006]

Active Update (rewriting)

$$
C \leftarrow \underbrace{+c_{1} p_{c} p_{c}{ }^{T}+c_{\mu} \sum_{i=1}^{\lfloor\lambda / 2\rfloor} w_{i} \boldsymbol{y}_{i: \lambda} \boldsymbol{y}_{i: \lambda}^{\mathrm{T}}}_{\text {increasing the variances in promising directions }}-\overbrace{c_{\mu} \sum_{i=\lambda-\lfloor\lambda / 2\rfloor+1}^{\lambda}\left|w_{i}\right| \boldsymbol{y}_{i: \lambda} \boldsymbol{y}_{i: \lambda}^{\mathrm{T}}}^{\text {decreasing the variances in unpromising directions }}
$$

- increases the variance in the directions of $p_{c}$ and promising steps $\boldsymbol{y}_{i: \lambda}(i \leq\lfloor\lambda / 2\rfloor)$

- decrease the variance in the directions of unpromising steps $\boldsymbol{y}_{i: \lambda}$ $(i \geq \lambda-\lfloor\lambda / 2\rfloor+1)$

- keep the variance in the subspace orthogonal to the above 


\section{On 10D Discus Function}

10D Discus Function (axis ratio: $\alpha=10^{3}$ )

$$
f(x)=\alpha^{2} \cdot x_{1}^{2}+\sum_{i=1}^{n} x_{i}^{2}
$$
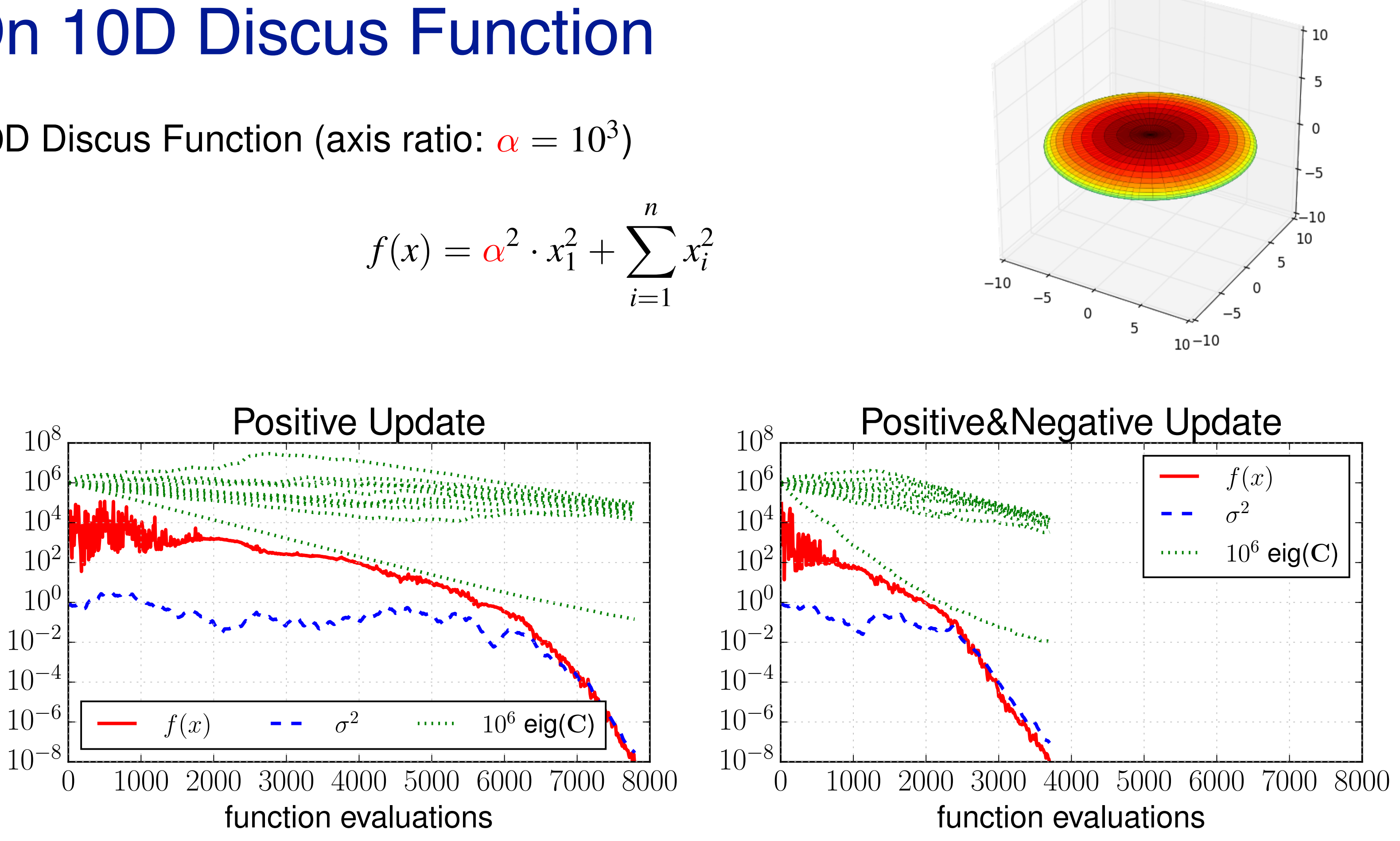

- Positive: wait for the smallest eig $(C)$ decreasing

- Active: decrease the smallest eig $(C)$ actively 


\section{Summary}

Active Covariance Matrix Adaptation + Cumulation

$C \leftarrow\left(1-c_{1}-c_{\mu}+c_{\mu}^{-}\right) C+c_{1} p_{c} p_{c}{ }^{T}+c_{\mu} \sum_{i=1}^{\lfloor\lambda / 2\rfloor} w_{i} \boldsymbol{y}_{i: \lambda} \boldsymbol{y}_{i: \lambda}^{\mathrm{T}}-c_{\mu}^{-} \sum_{i=\lambda-\lfloor\lambda / 2\rfloor+1}^{\lambda}\left|w_{i}\right| \boldsymbol{y}_{i: \lambda} \boldsymbol{y}_{i: \lambda}^{\mathrm{T}}$

- $-\left|w_{i}\right|<0$ (for $i \geq \lambda-\lfloor\lambda / 2\rfloor+1$ ): negative weight assigned to $\boldsymbol{y}_{i: \lambda}$, $\sum_{i=\lambda-\mu}^{\lambda}\left|w_{i}\right|=1$.

- $c_{\mu}^{-}>0$ : learning rate for the active update

These components complement each other

An important yet solvable issue of active update 
Input: $m \in \mathbb{R}^{n} ; \sigma \in \mathbb{R}_{+} ; \lambda \in \mathbb{N}_{\geq 2}$, usually $\lambda \geq 5$, default $4+\lfloor 3 \log n\rfloor$

Set $c_{m}=1 ; c_{1} \approx 2 / n^{2} ; c_{\mu} \approx \mu_{w} / n^{2} ; c_{\mathrm{c}} \approx 4 / n ; c_{\sigma} \approx 1 / \sqrt{n} ; d_{\sigma} \approx 1 ; w_{i=1 \ldots \lambda}$ decreasing in $i$ and $\sum_{i}^{\mu} w_{i}=1, w_{\mu}>0 \geq w_{\mu+1}, \mu_{w}^{-1}:=\sum_{i=1}^{\mu} w_{i}^{2} \approx 3 / \lambda$

Initialize $\mathrm{C}=\mathbf{I}$, and $p_{\mathrm{C}}=\mathbf{0}, p_{\sigma}=\mathbf{0}$

While not terminate

$$
\begin{array}{lr}
\boldsymbol{x}_{i}=m+\sigma \boldsymbol{y}_{i}, \quad \text { where } \boldsymbol{y}_{i} \sim \mathcal{N}_{i}(\mathbf{0}, \mathrm{C}) \text { for } i=1, \ldots, \lambda & \text { sampling } \\
m \leftarrow m+c_{m} \sigma \boldsymbol{y}_{w}, \text { where } \boldsymbol{y}_{w}=\sum_{i=1}^{\mu} w_{\operatorname{rk}(i)} \boldsymbol{y}_{i} & \text { update mean } \\
p_{\sigma} \leftarrow\left(1-c_{\sigma}\right) p_{\sigma}+\sqrt{1-\left(1-c_{\sigma}\right)^{2}} \sqrt{\mu_{w}} \mathrm{C}^{-\frac{1}{2}} \boldsymbol{y}_{w} & \text { path for } \sigma \\
p_{\mathrm{C}} \leftarrow\left(1-c_{\mathrm{C}}\right) p_{\mathrm{C}}+\mathbb{1}_{[0,2 n]}\left\{\left\|p_{\sigma}\right\|^{2}\right\} \sqrt{1-\left(1-c_{\mathrm{C}}\right)^{2}} \sqrt{\mu_{w}} \boldsymbol{y}_{w} & \text { path for } \mathrm{C} \\
\sigma \leftarrow \sigma \times \exp \left(\frac{c_{\sigma}}{d_{\sigma}}\left(\frac{\left\|p_{\sigma}\right\|}{\mathrm{E}\|\mathcal{N}(\mathbf{0}, \mathbf{I})\|}-1\right)\right) & \text { update of } \sigma \\
\mathbf{C} \leftarrow \mathbf{C}+c_{\mu} \sum_{i=1}^{\lambda} w_{\operatorname{rk}(i)}\left(\boldsymbol{y}_{i} \boldsymbol{y}_{i}^{\top}-\mathrm{C}\right)+c_{1}\left(p_{\mathrm{c}} p_{\mathrm{C}}^{\top}-\mathrm{C}\right) & \text { update C }
\end{array}
$$

Not covered: termination, restarts, useful output, search boundaries and encoding, corrections for: positive definiteness guaranty, $p_{\mathrm{c}}$ variance loss, $c_{\sigma}$ and $d_{\sigma}$ for large $\lambda$ 


\section{Topics}

3. What can/should the users do for the CMA-ES to work effectively on their problem?

- Choice of problem formulation and encoding (not covered)

- Choice of initial solution and initial step-size

- Restarts, Increasing Population Size

- Restricted Covariance Matrix 


\section{Default Parameter Values}

CMA-ES + (B)IPOP Restart Strategy $=$ Quasi-Parameter Free Optimizer

The following parameters were identified in carefully chosen experimental set ups.

- related to selection and recombination

- $\lambda$ : offspring number, new solutions sampled, population size

- $\mu$ : parent number, solutions involved in mean update

- $w_{i}$ : recombination weights

- related to $C$-update

- $1-c_{c}$ : decay rate for the evolution path, cumulation factor

- $c_{1}$ : learning rate for rank-one update of $C$

- $c_{\mu}$ : learning rate for rank- $\mu$ update of $C$

- related to $\sigma$-update

- $1-c_{\sigma}$ : decay rate of the evolution path

- $d_{\sigma}$ : damping for $\sigma$-change

The default values depends only on the dimension. They do in the first place not depend on the objective function. 


\section{Parameters to be set depending on the problem}

Initialization and termination conditions

The following should be set or implemented depending on the problem.

- related to the initial search distribution

- $m^{(0)}$ : initial mean vector

- $\sigma^{(0)}$ (or $\sqrt{C_{i, i}^{(0)}}$ ): initial (coordinate-wise) standard deviation

- related to stopping conditions

- max. func. evals.

- max. iterations

- function value tolerance

- min. axis length

- stagnation

Practical Hints:

- start with an initial guess $m^{(0)}$ with a relatively small step-size $\sigma^{(0)}$ to locally improve the current guess;

- then increase the step-size, e.g., by factor of 10 , to globally search for a better solution. 


\section{Python CMA-ES Implementation}

\section{https://github.com/CMA-ES/pycma}

\section{pycma}

A Python implementation of CMA-ES and a few related numerical optimization tools.

The Covariance Matrix Adaptation Evolution Strategy (CMA-ES) is a stochastic derivative-free numerical optimization algorithm for difficult (non-convex, ill-conditioned, multi-modal, rugged, noisy) optimization problems in continuous search spaces.

Useful links:

- A quick start guide with a few usage examples

- The API Documentation

- Hints for how to use this (kind of) optimization module in practice

\section{Installation of the latest release}

Type

python -m pip install cma

in a system shell to install the latest release from the Python Package Index (PyPI). The release link also provides more installation hints and a quick start guide. 


\section{Python CMA-ES Demo}

\section{https://github.com/CMA-ES/pycma}

\section{Optimizing 10D Rosenbrock Function}

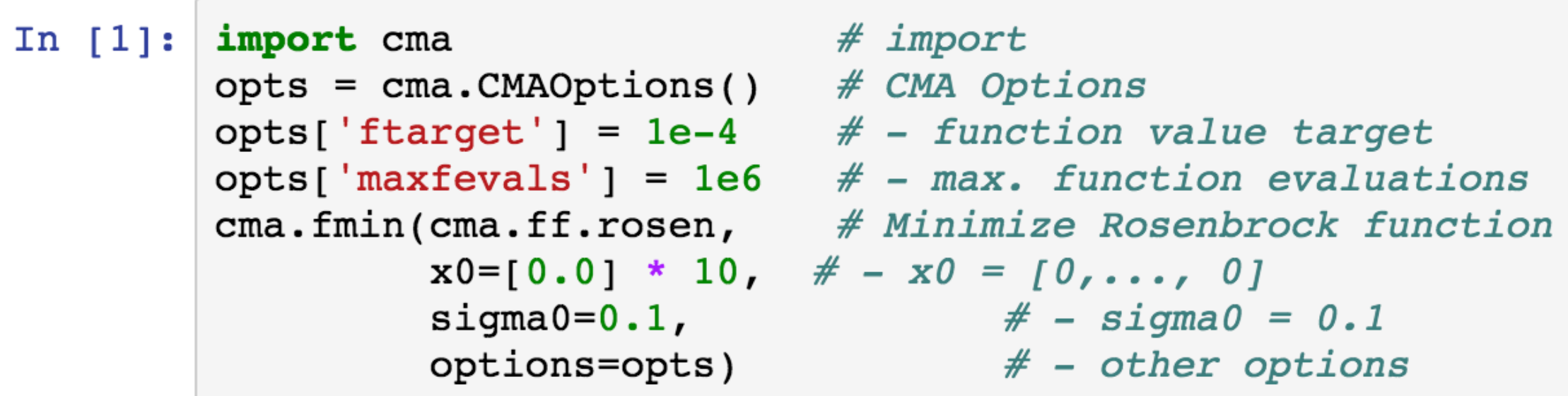

$\left(5 \_\mathrm{w}, 10\right)$-aCMA-ES (mu_w=3.2, w_1 $=45 \%$ ) in dimension 10 (seed=909490, Mon Ap r $16 \quad 13: 39: 57$ 2018)

Iterat \#Fevals function value axis ratio sigma min\&max std $t[\mathrm{~m}: \mathrm{s}]$

\begin{tabular}{|c|c|c|c|c|c|c|c|}
\hline 1 & 10 & +01 & $1.0 e+00$ & $9.12 \mathrm{e}-02$ & $9 e-02$ & $9 e-02$ & $0: 00.0$ \\
\hline 2 & 20 & $1.363303277917634 \mathrm{e}+01$ & $1.1 e+00$ & $8.33 e-02$ & $8 e-02$ & $8 e-02$ & $0: 00.0$ \\
\hline 3 & 30 & $9892 e+01$ & $2 e+00$ & 2 & 2 & 02 & 0.0 \\
\hline 0 & 1000 & +00 & 00 & 2 & 3 & 02 & 1 \\
\hline 00 & 2000 & $550841127554589 e+00$ & $1.5 e+01$ & $3.97 e-02$ & $1 e-02$ & $e-02$ & 00.2 \\
\hline 300 & 3000 & $3.674986141687857 e-01$ & $1.5 e+01$ & $2.76 e-02$ & $3 e-03$ & $2 e-02$ & )$: 00.4$ \\
\hline 00 & 4000 & $.266345464781239 e-03$ & $5.0 e+01$ & $1.18 e-02$ & $8 e-04$ & $2 e-02$ & $: 00.5$ \\
\hline 20 & 4200 & $.039461687999381 e-05$ & $5.5 e+01$ & $4.04 e-03$ & $2 e-04$ & $5 e-03$ & )$: 00.5$ \\
\hline
\end{tabular}
termination on ftarget $=0.0001$ (Mon Apr 16 13:39:58 2018)

final/bestever f-value $=2.804423 \mathrm{e}-052.804423 \mathrm{e}-05$

incumbent solution: $\left[\begin{array}{lllll}0.9998542 & 0.99996219 & 0.9999681 & 1.00000445 & 0 .\end{array}\right.$ $99998977 \quad 0.99968537$

$0.99954974 \quad 0.99918266 \ldots]$

std deviations: [ $\left[\begin{array}{llllll}0.00023937 & 0.00022203 & 0.00024836 & 0.00024782 & 0.0003\end{array}\right.$

$1258 \quad 0.00043481$

$0.000782610 .0014964 \quad \ldots]$

:tive:

isation

tend to do is itial) solution a. Then I (can) vely (by a sending on iitial usly) and see or better (or 


\section{Python CMA-ES Demo}

\section{https://github.com/CMA-ES/pycma}

\section{Optimizing 10D Rosenbrock Function}

\section{cma.plot( )}

Figure 325

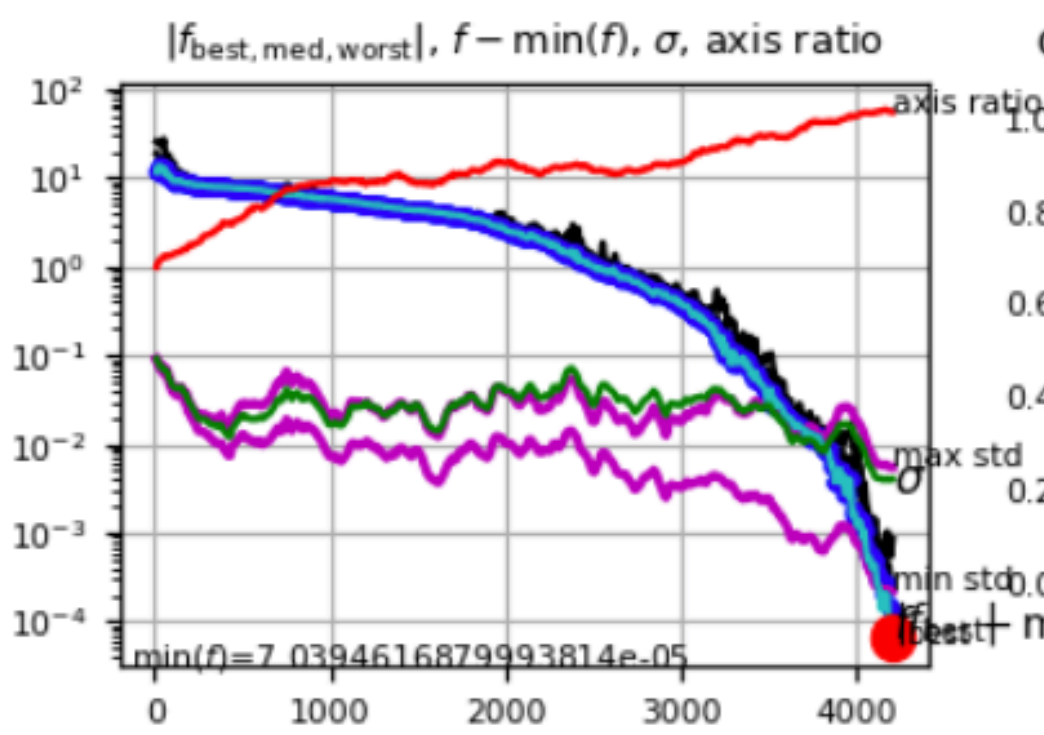

Object Variables (curr best, 10-D, popsize 10)
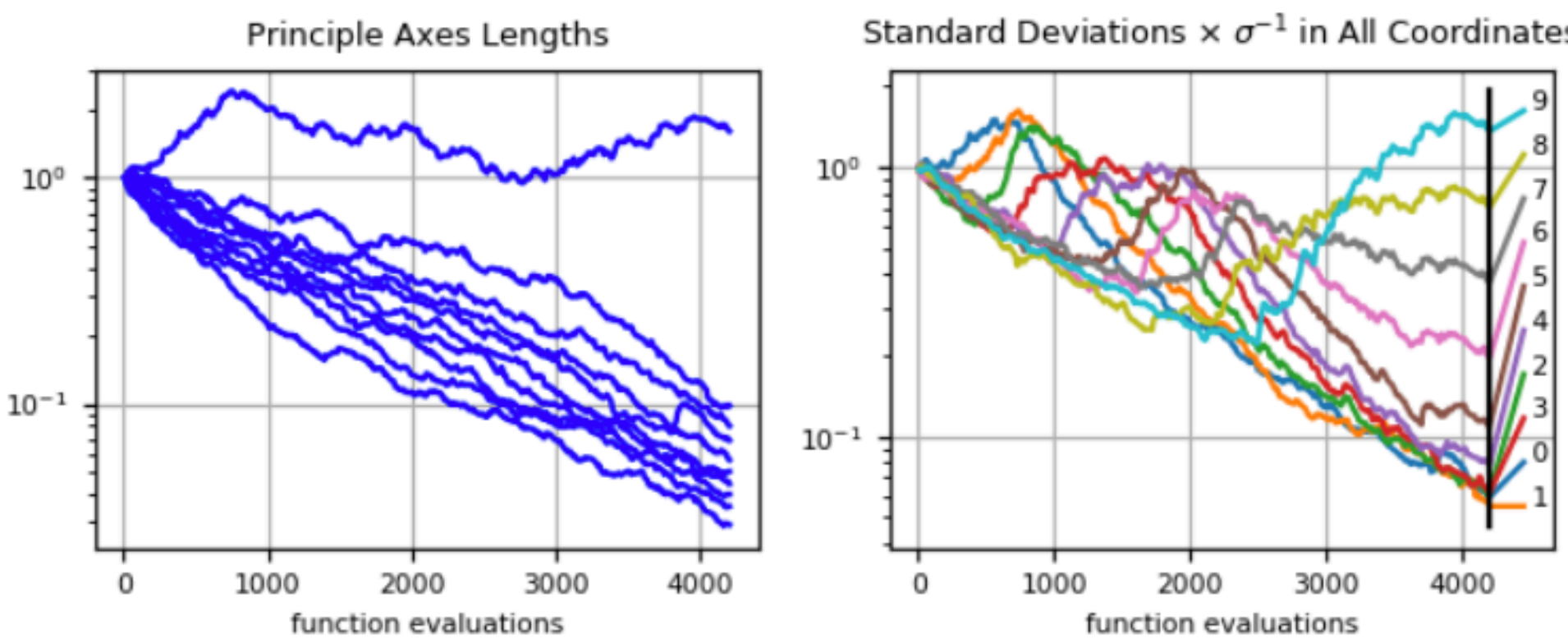


\section{Multimodality}

Two approaches for multimodal functions: Try again with

- a larger population size

- a smaller initial step-size (and random initial mean vector) 


\section{Multimodality}

Approaches for multimodal functions: Try again with

- the final solution as initial solution (non-elitist) and small step-size

- a larger population size

- a different initial mean vector (and a smaller initial step-size)

A restart with a large population size helps if the objective function has a well global structure

- functions such as Schaffer, Rastrigin, BBOB function 15 19

- loosely, unimodal global structure + deterministic noise
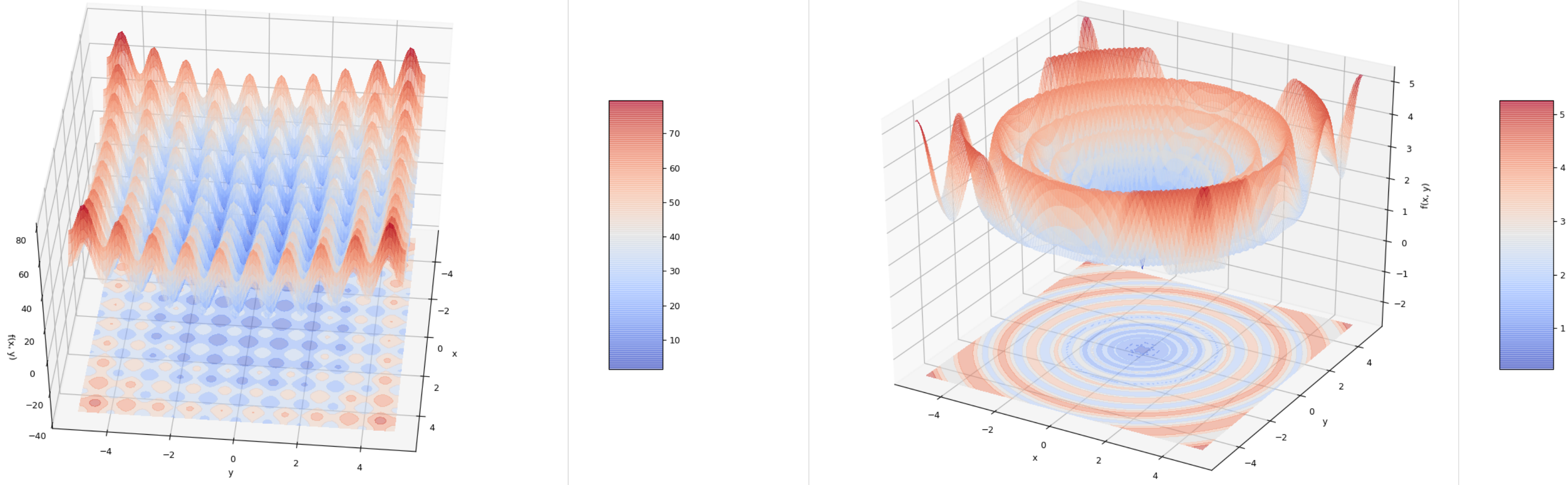


\section{Multimodality}

Hansen and Kern. Evaluating the CMA Evolution Strategy on Multimodal Test Functions, PPSN 2004.

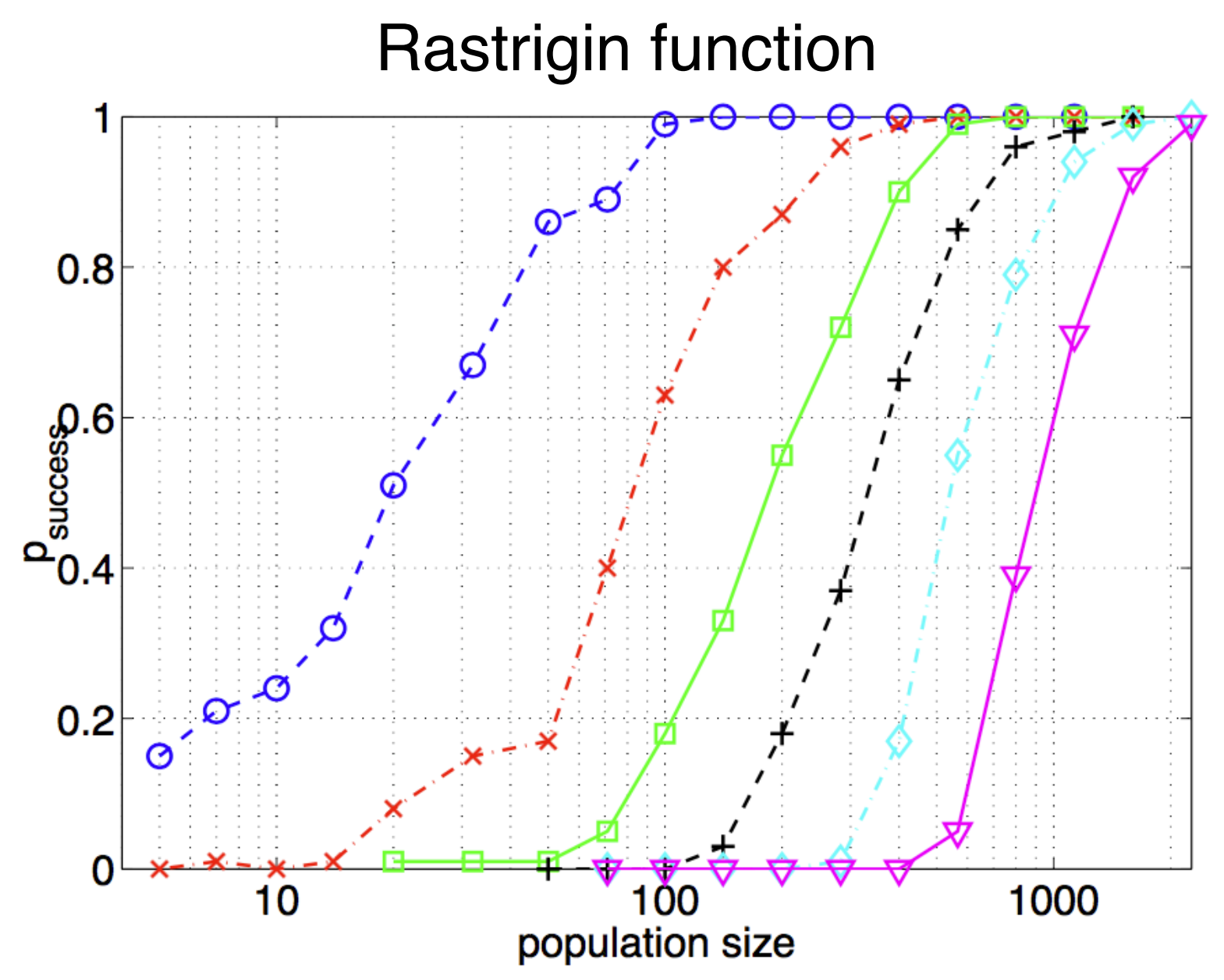

(a)

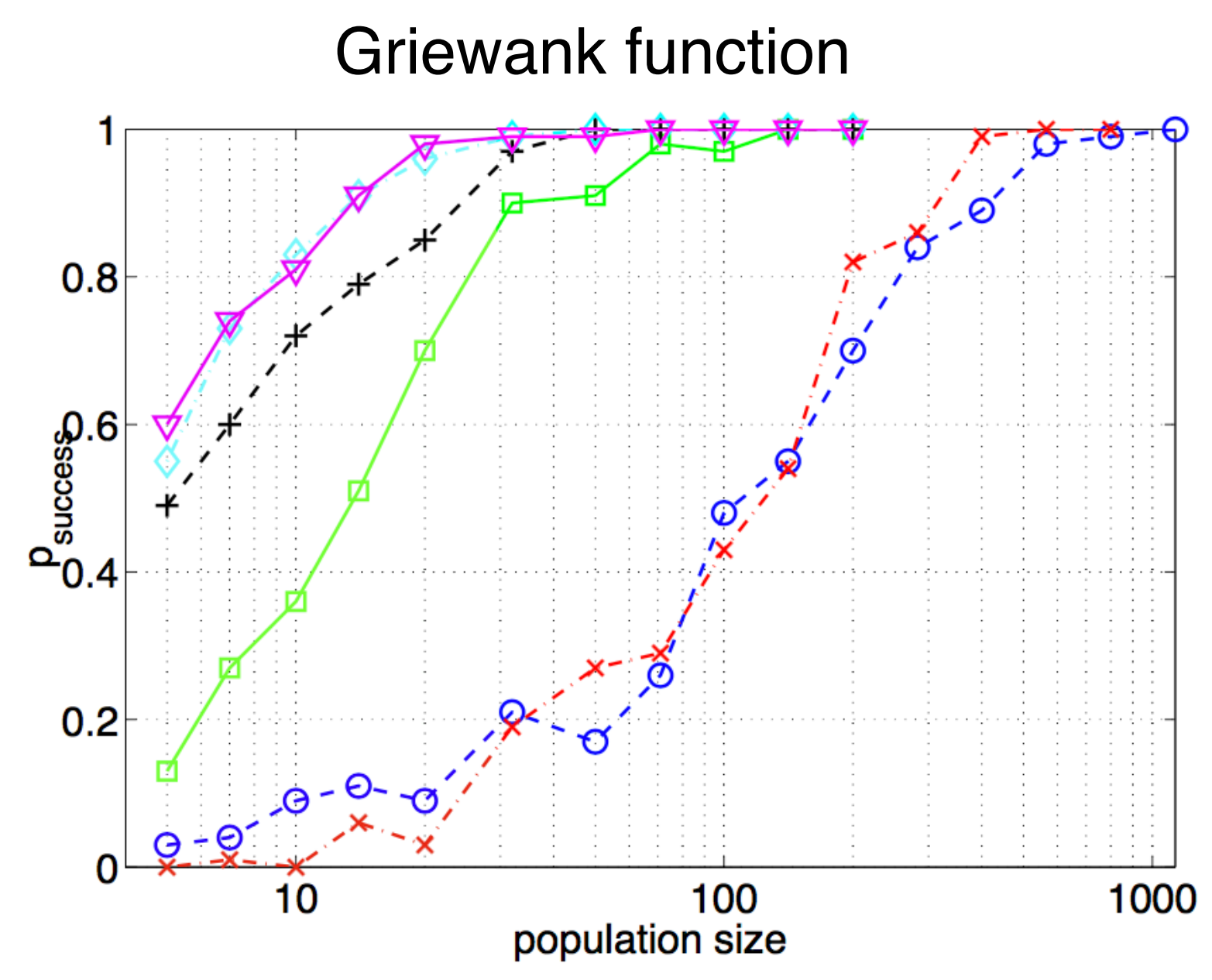

(b)

Fig. 1. Success rate to reach $f_{\text {stop }}=10^{-10}$ versus population size for (a) Rastrigin function (b) Griewank function for dimensions $n=2('--\bigcirc--$ '), $n=5($ ' $-\cdot-\times-\cdot-$ '),

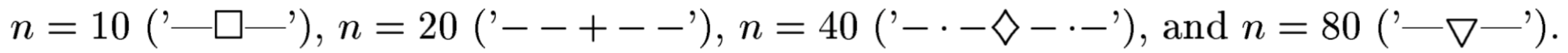




\section{Multimodality}

Approaches for multimodal functions: Try again with

- the final solution as initial solution (non-elitist) and small step-size

- a larger population size

- a different initial mean vector (and a smaller initial step-size)

A restart with a small initial step-size helps if the objective function has a weak global structure

- functions such as Schwefel, Bi-Sphere, BBOB function 20 24
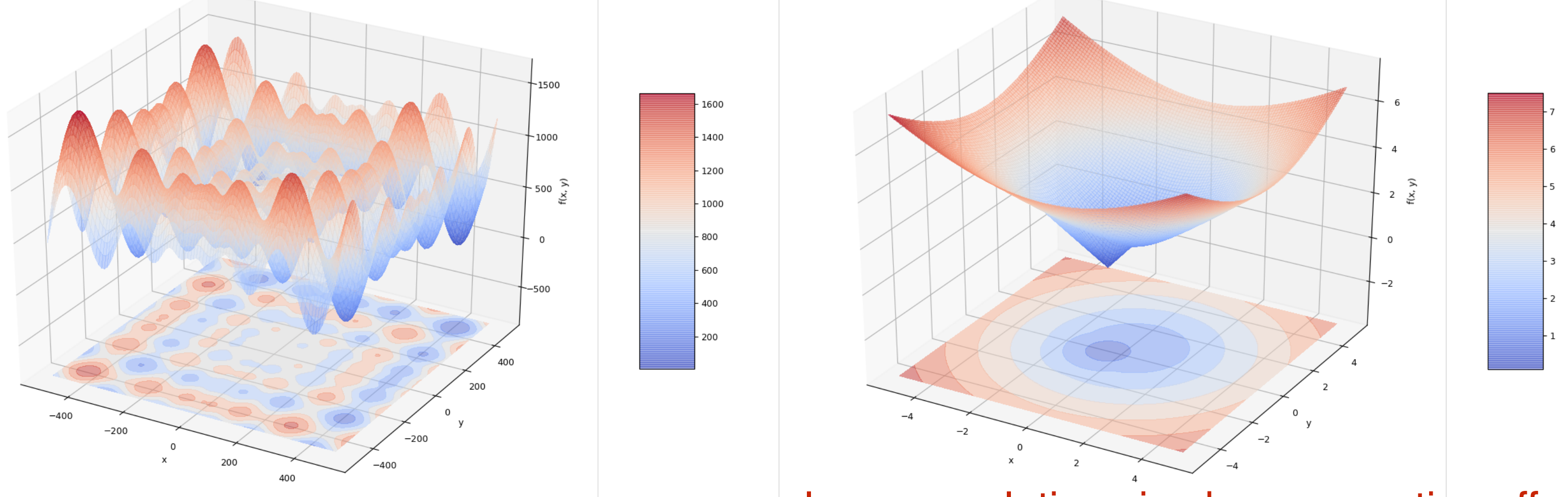


\section{Restart Strategy}

It makes the CMA-ES parameter free

IPOP: Restart with increasing the population size

- start with the default population size

- double the population size after each trial (parameter sweep)

- may be considered as gold standard for automated restarts

BIPOP: IPOP regime + Local search regime

- IPOP regime: restart with increasing population size

- Local search regime: restart with a smaller step-size and a smaller population size than the IPOP regime 


\section{Topics}

3. What can/should the users do for the CMA-ES to work effectively on their problem?

- Choice of problem formulation and encoding (not covered)

- Choice of initial solution and initial step-size

- Restarts, Increasing Population Size

- Restricted Covariance Matrix 


\section{Motivation of the Restricted Covariance Matrix}

Bottlenecks of the CMA-ES on high dimensional problems

(1) $\mathcal{O}\left(N^{2}\right)$ Time and Space Complexities

- to store and update $C \in \mathbb{R}^{N \times N}$

- to compute the eigen decomposition of $C$ 


\section{Variants with Restricted Covariance Matrix}

\section{CMA-ES Variants with Restricted Covariance Matrices}

- Sep-CMA [Ros and Hansen, 2008]

- $C=$ D. D: Diagonal

- VD-CMA [Akimoto et al., 2014]

- $C=\mathbf{D}\left(\mathbf{I}+\boldsymbol{v} \boldsymbol{v}^{\mathrm{T}}\right) \mathbf{D}$. D: Diagonal, $\boldsymbol{v} \in \mathbb{R}^{N}$.

- LM-CMA [Loshchilov, 2014]

- $C=\mathbf{I}+\sum_{i=1}^{k} \boldsymbol{v}_{i} \boldsymbol{v}_{i}^{\mathrm{T}} \cdot \boldsymbol{v}_{i} \in \mathbb{R}^{N}$.

- VkD-CMA [Akimoto and Hansen, 2016]

- $C=\mathbf{D}\left(\mathbf{I}+\sum_{i=1}^{k} \boldsymbol{v}_{i} \boldsymbol{v}_{i}^{\mathrm{T}}\right) \mathbf{D} . \boldsymbol{v}_{i} \in \mathbb{R}^{N}$.

[Ros and Hansen, 2008] Ros, R. and Hansen, N. (2008). A simple modification in CMA-ES achieving linear time and space complexity. In Parallel Problem Solving from Nature - PPSN X, pages 296-305. Springer.

[Akimoto et al., 2014] Akimoto, Y., Auger, A., and Hansen, N. (2014). Comparison-based natural gradient optimization in high dimension. In Proceedings of Genetic and Evolutionary Computation Conference, pages 373-380, Vancouver, BC, Canada.

[Loshchilov, 2014] Loshchilov, I. (2014). A computationally efficient limited memory cma-es for large scale optimization. In Proceedings of Genetic and Evolutionary Computation Conference, pages 397-404.

[Akimoto and Hansen, 2016] Akimoto, Y. and Hansen, N. (2016). Projection-based restricted covariance matrix adaptation for high dimension. In Genetic and Evolutionary Computation Conference, GECCO 2016, Denver, Colorado, USA, July 20-24, 2016, page (accepted). ACM. 


\section{Separable CMA (Sep-CMA)}

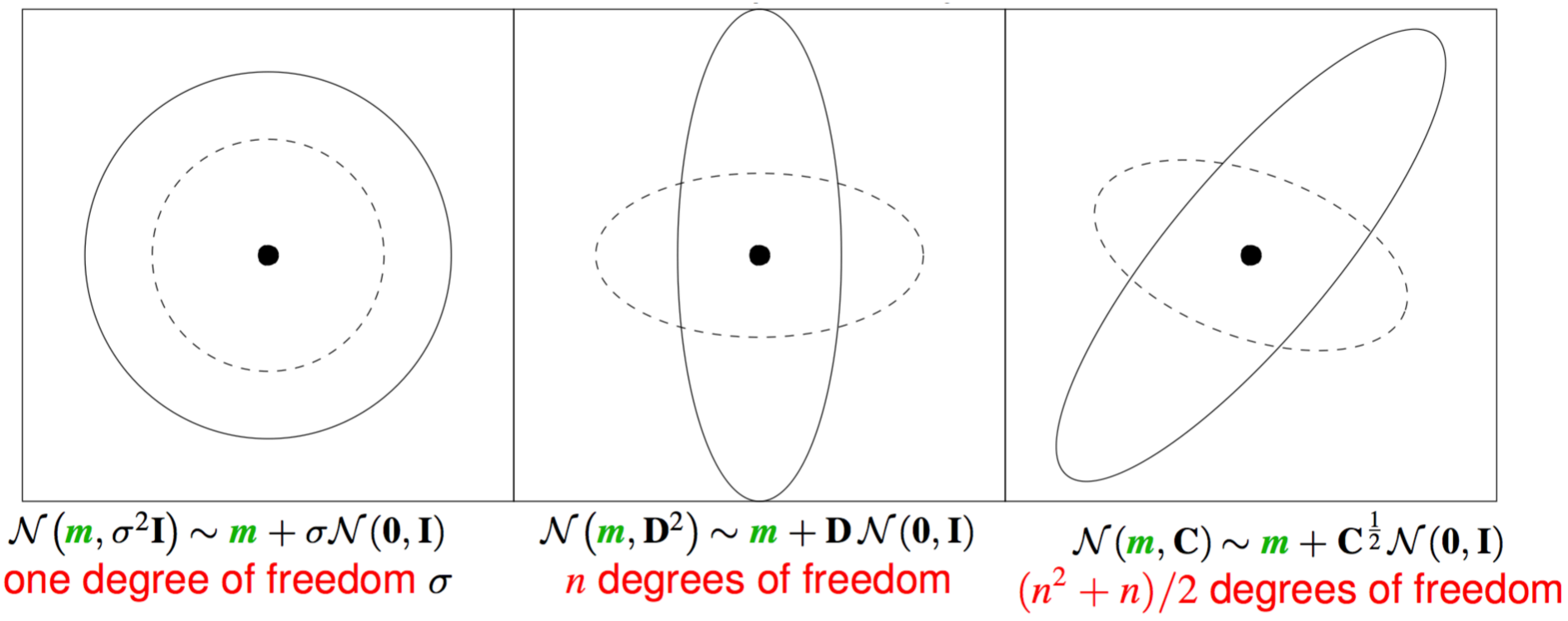

$\mathrm{CMA} \quad C_{\mathrm{cma}}^{(t+1)}=C^{(t)}+c_{1}\left(p_{c} p_{c}{ }^{\mathrm{T}}-C^{(t)}\right)+c_{\mu} \sum_{i=1}^{\mu} w_{i}\left(\left(\boldsymbol{x}_{i}-m^{(t)}\right)\left(\boldsymbol{x}_{i}-m^{(t)}\right)^{\mathrm{T}}-C^{(t)}\right)$

SEP $\left[C_{\text {sep }}^{(t+1)}\right]_{k, k}=\left[C^{(t)}\right]_{k, k}+\underset{T}{c_{1}}\left(\left[p_{c}\right]_{k}^{2}-\left[C^{(t)}\right]_{k, k}\right)+\underset{c_{\mu}}{c_{i=1}^{\mu}} w_{i}\left(\left[\boldsymbol{x}_{i}-m^{(t)}\right]_{k}^{2}-\left[\boldsymbol{C}^{(t)}\right]_{k, k}\right)$

$(\mathrm{N}+2) / 3$ times greater than CMA 


\section{Demo: On 100D Separable Ellipsoid Function}

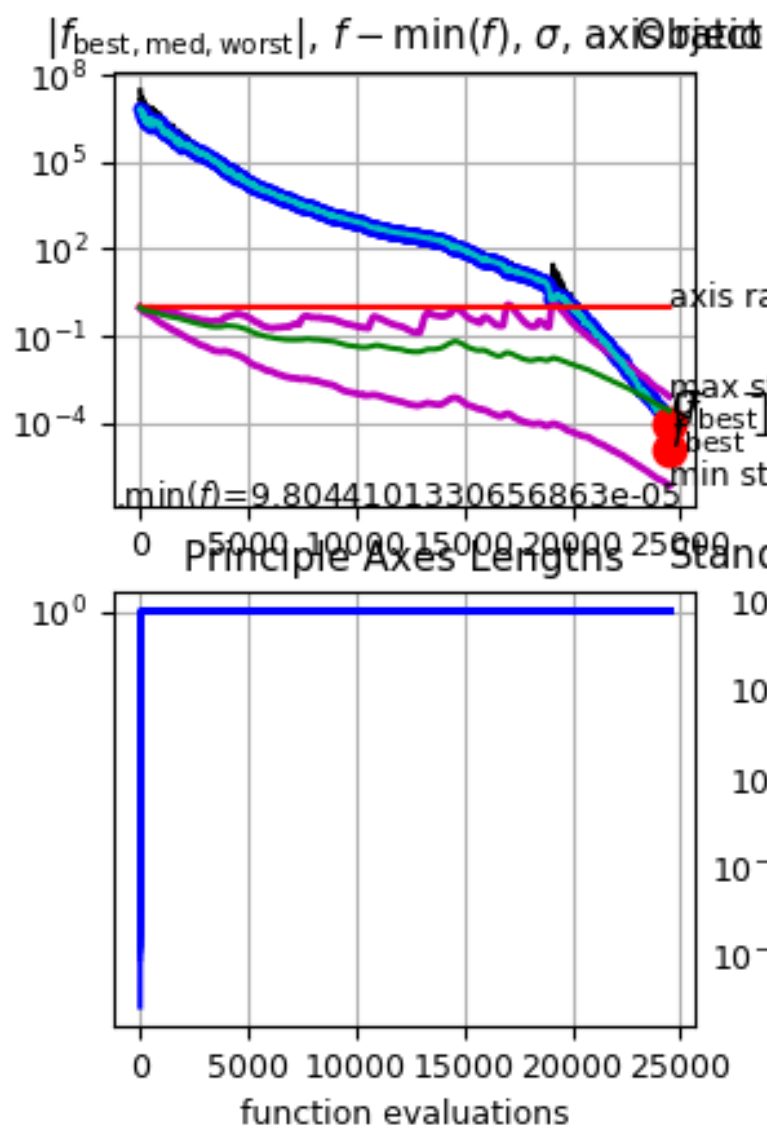

Separable-CMA $\left|f_{\text {best, med, worst }}\right|, f-\min (f), \sigma$, axielbatad Variables (curr best, 100-D, popsize $\sim 17$.

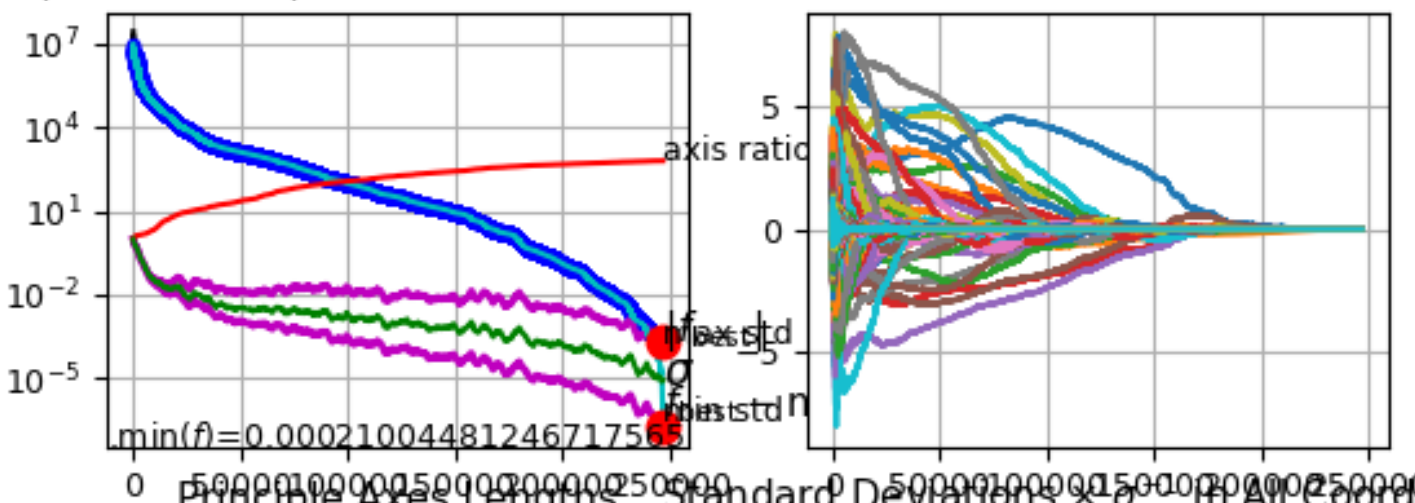

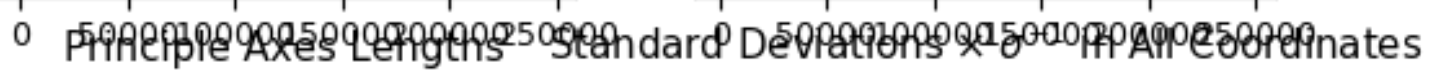
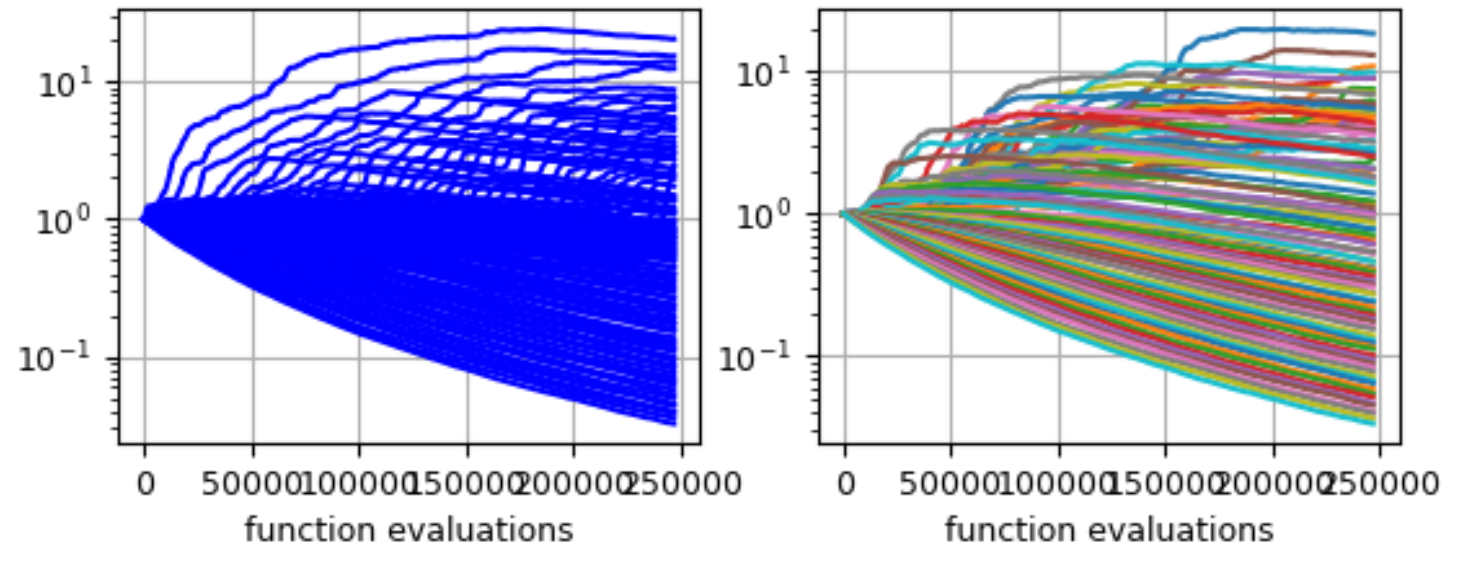

CMA

- CMA needed 10 times more FEs + more CPU time

- However, Sep-CMA won't be able to solve rotated ellipsoid function as efficiently as it solves separable ellipsoid 


\section{Summary and Final Remarks}




\section{Main Characteristics of (CMA) Evolution Strategies}

- Multivariate normal distribution to generate new search points follows the maximum entropy principle

(2) Rank-based selection

implies invariance, same performance on $g(f(\boldsymbol{x}))$ for any increasing $g$ more invariance properties are featured

(3) Step-size control facilitates fast (log-linear) convergence and possibly linear scaling with the dimension

in CMA-ES based on an evolution path (a non-local trajectory)

(4) Covariance matrix adaptation (CMA) increases the likelihood of previously successful steps and can improve performance by orders of magnitude

the update follows the natural gradient

$\mathrm{C} \propto \boldsymbol{H}^{-1} \Longleftrightarrow$ adapts a variable metric $\Longleftrightarrow$ new (rotated) problem representation $\Longrightarrow f: \boldsymbol{x} \mapsto g\left(\boldsymbol{x}^{\mathrm{T}} \boldsymbol{H} \boldsymbol{x}\right)$ reduces to $\boldsymbol{x} \mapsto \boldsymbol{x}^{\mathrm{T}} \boldsymbol{x}$ 


\section{Limitations}

of CMA Evolution Strategies

- internal CPU-time: $10^{-8} n^{2}$ seconds per function evaluation on a $2 \mathrm{GHz}$ PC, tweaks are available

$1000000 f$-evaluations in 100-D take 100 seconds internal CPU-time

variants with restricted covariance matrix such as Sep-CMA

- better methods are presumably available in case of

- partly separable problems

- specific problems, for example with cheap gradients

specific methods

- small dimension $(n \ll 10)$

for example Nelder-Mead

- small running times (number of $f$-evaluations $<100 n$ ) model-based methods 


\section{Thank you}

Source code for CMA-ES in C, $\mathrm{C}++$, Java, Matlab, Octave, Python, R, Scilab and

Practical hints for problem formulation, variable encoding, parameter setting are available (or linked to) at

http://cma.gforge.inria.fr/cmaes_sourcecode_page.html 


\section{Comparison during BBOB at GECCO 2010}

\section{4 functions and 20+ alaorithms in 20-D}

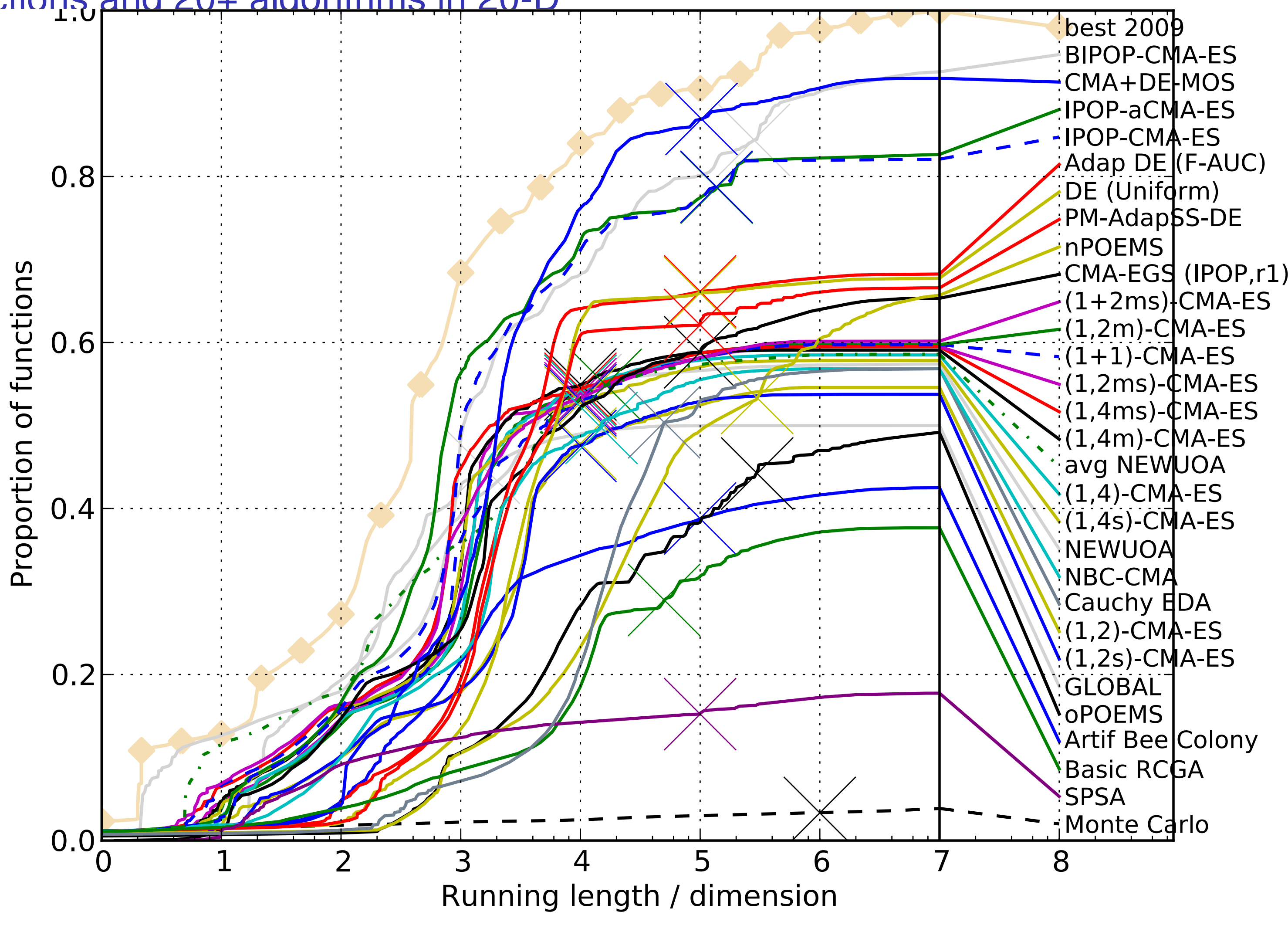

